\title{
IMPLEMENTING PARAPROFESSIONAL STRENGTH-BASED EARLY INTERVENTION HOME VISITATIONS
}

\author{
A Thesis Submitted to the College of \\ Graduate Studies and Research \\ in Partial Fulfillment of the Requirements \\ for the Degree of Doctor of Philosophy \\ in the Department of Educational Psychology and Special Education \\ University of Saskatchewan \\ Saskatoon
}

By

David Brian Mykota

Fall, 2002

C Copyright David Brian Mykota, 2002. All rights reserved 


\section{PERMISSION TO USE}

In presenting this thesis in partial fulfillment of the requirements for a Postgraduate degree from the University of Saskatchewan, I agree that the Libraries of this University may make it freely available for inspection. I further agree that permission for copying of this thesis in any manner, in whole or in part, for scholarly purposes may be granted by the professor or professors who supervised my thesis work or, in their absence, by the Head of the Department or the Dean of the College in which my thesis work is done. It is understood that any copying, publication, or use of this thesis or parts thereof for financial gain shall not be allowed without my written permission. It is also understood that due recognition shall be given to me and to the University of Saskatchewan in any scholarly use which may be made of any material in my thesis.

Requests for permission to copy or to make other use of material in this thesis in whole or part should be addressed to:

Head of the Department of Educational Psychology and Special Education

University of Saskatchewan

Saskatoon, Saskatchewan. S7N 0X1 


\begin{abstract}
The purpose of the present study is to evaluate the implementation process for Parenting Plus, the early intervention program of the Pipestone Health District. Parenting Plus, as modeled after Hawaii Healthy Start, provides strength-based paraprofessional home visitations to overburdened parents of newborns. The goal of the program is to strengthen families through the personal development of young parents that includes their ability to use community-based resources to affect positive parenting outcomes.
\end{abstract}

A utilization-focused evaluation involving stakeholders that followed an implementation framework was designed. In so doing, the objectives of the study were threefold. The first goal was to understand the model of service delivery that has emerged and been operationalized for Parenting Plus. The second aim was to explore the characteristics of the implementation process that have facilitated or hindered the development of Parenting Plus. The third intention was to gain insight into what the parent's experience and understand about the paraprofessional home visitations they receive from Parenting Plus.

A mixed methods approach to program evaluation was used that incorporated the general inductive approach. The quantitative methods for data collection included a document review and parent completion of the Family-Centered Program Rating Scale (FamPRS). The qualitative methods for data collection included semi-structured depth interviews and focus groups. The general inductive approach to data analysis resulted in five major dimensions that support a model for understanding the implementation and operationalization of paraprofessional strength-based home visitations. 
Recommendations for policy and practice address the need for intersectoral involvement as being crucial to a strength-based pilot project's success. In addition, more time needs to be allocated, prior to program implementation, for partnership building with stakeholders and the public. Thus, public education and awareness surrounding the program model's feasibility and applicability would aid in alleviating misconceptions and misunderstandings, build partnerships, and facilitate program implementation among stakeholders. Future research needs to not only look at the mode of service delivery but, more importantly, at how the characteristics of the home visitor can effect change in the participant and what level of experience or education in the paraprofessional is best suited to a particular client population. 


\section{ACKNOWLEDGEMENTS}

I would like to acknowledge the financial assistance received through the Pipestone Health District for the completion of the present study. In particular, I would like to thank my committee member Dr. Joe Kluger from Saskatchewan Health, for engendering partnerships between the University and the health district that enabled the present project to come to fruition. I would like to thank my external examiner Dr. Henry Janzen from the University of Alberta for his thorough and critical review of my work. I would also like to thank committee members Dr. Fred Reekie, Dr. Don Saklofske, and Dr. Keith Walker for their comments and insight that allowed me to critically reflect on the dissertation. I would like to acknowledge my advisor, Dr. Vicki Schwean for encouraging me to enter the department's doctoral program and for her continued commitment to research excellence.

A special thanks to Nancy Cochrane who unselfishly devoted considerable time and effort, as editor, to expeditiously bring the project to closure. I would also like to thank Dr. David Thomas from the University of Auckland, New Zealand for his advice and guidance in conducting qualitative analysis for program evaluations. In conclusion, this study would not have been possible without the support and co-operation of the Pipestone Health District and Parenting Plus, thank you Ronell, Linda, Crystal, and Heather. Finally, I would like to express my sincere gratitude to all the families who participated in the study and gave of themselves, their contribution was invaluable to the evaluation undertaken. 


\section{DEDICATION}

This dissertation is dedicated in the memory

of

Richard Alan (Al) Yackulic

$1947-2002$

Department of Educational Psychology and Special Education

College of Education

University of Saskatchewan 


\section{TABLE OF CONTENTS}

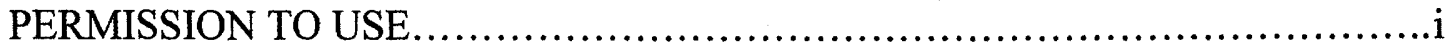

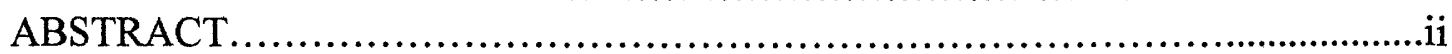

ACKNOWLEDGEMENTS ..........................................................

DEDICATION ......................................................................

TABLE OF CONTENTS .................................................................

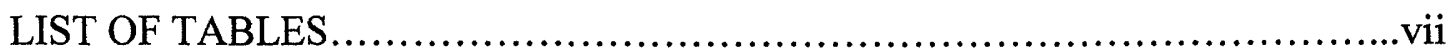

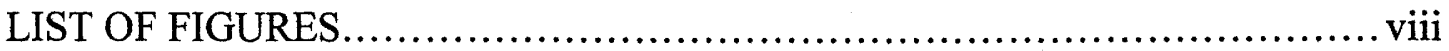

1 INTRODUCTTION..........................................................

Overview...............................................................

Purpose ......................................................................

Target Audience................................................................

Evaluation Framework............................................... 4

Research Questions................................................. 5

Research Question 1...................................... 5

Research Question 2 ..................................... 5

Research Question 3........................................ 5

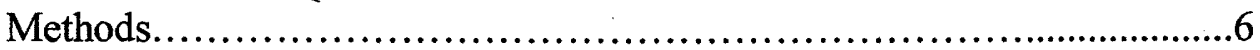

Assumptions........................................................6

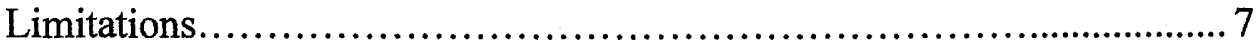

Delimitations......................................................... 7

Significance........................................................... 8

$2 \quad$ LITERATURE REVIEW ................................................. 9

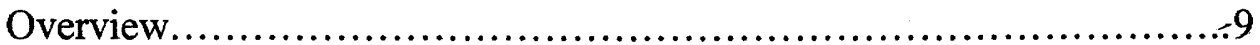

Early Intervention Theoretical Frameworks............................. 9

Home Visiting Programs...............................................17

Child Abuse and Neglect.............................................. 33

Hawaii Healthy Start.............................................. 37

Parenting Plus...................................................... 48

Program Rationale..................................................... 49

Program Description........................................ 50

Program Screening.................................................... 51

Program Delivery ..........................................5 53

Program Logic Model.......................................55

Strength-Based Model............................... 57

Program Resources........................................ 58

Expected Outcomes........................................ 59

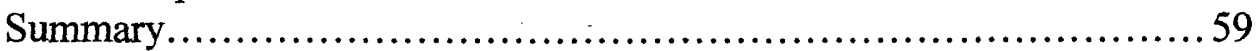

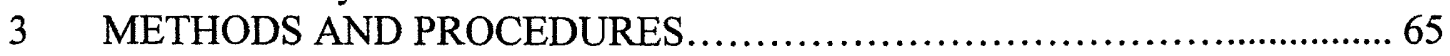

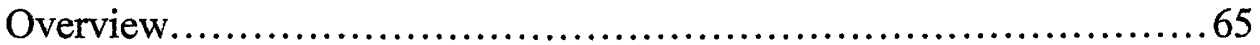

Evaluation Framework Rationale.......................................69 69

Implementation Evaluation.......................................72

Methodological Rationale.......................................... 75

Interviews...................................................... 82

Focus Groups.................................................... 93 
Empowerment Evaluation...............................95

Family-Centered Program Rating Scale.......................... 97

Document Review...............................................100

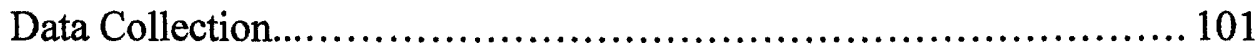

Interview Data Collection....................................... 101

Focus Group Data Collection.........................................106

FamPRS Data Collection....................................... 107

Document Review Data Collection............................. 107

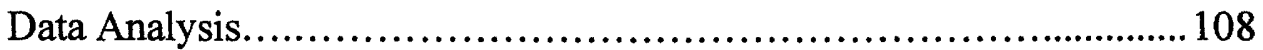

Summary............................................................... 111

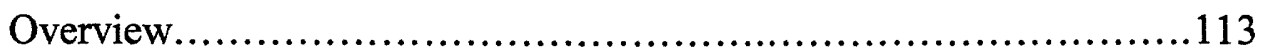

Research Questions.............................................115

Research Question \#1 ............................................... 115

Research Question \#2.......................................... 115

Research Question \#3......................................... 115

Research Question Results...........................................115

Program Status................................................ 112

Interview Results.......................................... 129

Partnership Building and Communication..............130

Screening and Assessment Concerns...................135

Challenges to Parenting Plus........................... 143

Lessons Learned.........................................149

Focus Group Results....................................... 155

Inductive Analysis of Focus Group Results..............160

Family-Centered Program Rating Scale Results..................165

Summary ....................................................... 170

Screening and Assessment........................................170

Home Visitations.......................................... 172

Linkages between Family and Health Care Community.............. 174

Co-ordination of Services and Supports......................... 176

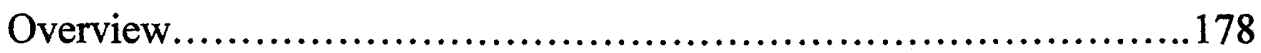

Findings.................................................................. 180

Effective Practices..................................................... 182

Screening and Assessment......................................... 187

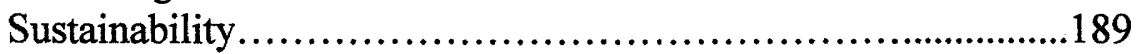

Strength-Based Home Visitations.......................................191

Research Implications.............................................. 194

Targeted Early Interventions...................................194

Paraprofessional Home Visitations...............................195

Implementation Evaluation...................................196

Short-term Outcome Evaluation.................................. 197

Longitudinal Research....................................... 198

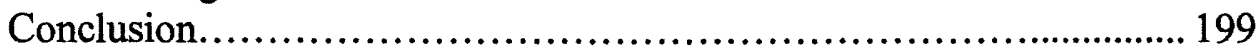

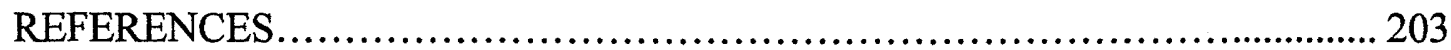




\section{APPENDIXES}

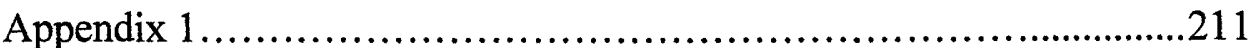

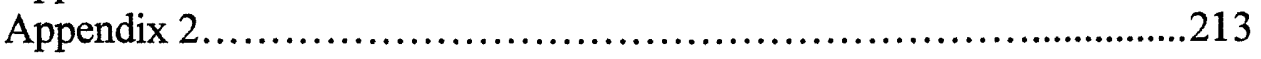

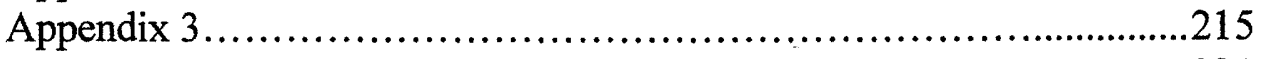

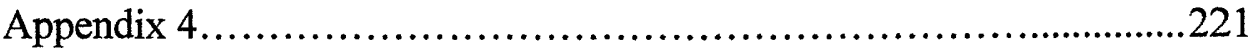

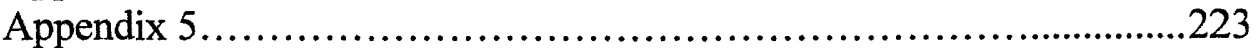

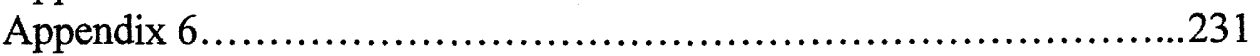

\section{LIST OF TABLES}

Table 1.1

Table 2.1

Table 2.2

Table 3.1

Table 3.2

Table 3.3

Table 3.4

Table 3.5

Table 3.6

Table 4.1

Table 4.2

Table 4.3

Table 4.4

Table 4.5

Table 4.6

Table 4.7

Table 4.8
Characteristics of Families at Various Levels of Psychosocial

Risk.........................................................2

Pipestone Health District Socioeconomic Indicators..............51

Parenting Plus Two-Stage Early Identification

Screening........................................................52

Underlying Assumptions of the General Inductive

Approach.........................................................78

Five Key Features of Coding Categories.......................8 80

Example of Coding Category...................................81

Research Questions and Interview Questions...................85

Original and Adapted Items of the FamPRS Parent's

Scale......................................................... 100

Interview Data Collection Schedule........................... 105

Parenting Plus Screening Tracking Summary................. 116

Parenting Plus Status of Screen.................................... 117

Participant Demographic Profile...............................122

Focus Group Data: Parenting Plus Mission Statement........... 157

Focus Group Data: Parenting Plus Key Activities.................157

Focus Group Data: Parenting Plus Prioritized Program

Activity Ratings................................................. 158

Focus Group Data: Parenting Plus Goals.........................159

FamPRS Anecdotal Comments....................................169 


\section{LIST OF FIGURES}

Figure 2.1

Figure 2.2

Figure 2.3

Figure 2.4

Figure 2.5

Figure 3.1

Figure 4.1

Figure 4.2

Figure 4.3

Figure 4.4

Figure 4.5

Figure 4.6

Figure 4.7

Figure 4.8

Figure 4.9

Figure 4.10

Figure 4.11

Figure 4.12

Figure 4.13

Figure 5.1

Figure 5.2
The 3-R's of Early Intervention Within a Transactional

Model.

Factors Influencing Children's Developmental

Outcomes.......................................................... 14

Components of Early Intervention.....................................15

Resource-Based Model of Early Intervention......................17

Parenting Plus Logic Model.......................................55

Relevance of the CIPP Model to Decision Making and

Accountability.... 74

Parenting Plus Program Status: October 2000..............................119

Birth Mother Family Stress Checklist Score:

Items 1-5.

Birth Mother Family Stress Checklist Score:

Items 6-10....

Birth Father Family Stress Checklist Score:

Items 1-5.

Birth Father Family Stress Checklist Score:

Items 6-10.

Length of Time in Program, Parenting Plus:

October 2000.

Home Visit Frequency by Length in Program,

Parenting Plus: October 2000...................................... 128

Partnership Building and Communication Dimension............ 131

Screening and Assessment Concerns Dimension................. 136

Challenges to Parenting Plus Dimension.......................... 144

Lessons Learned Dimension...................................... 150

Characteristics of Strength-Based Home Visiting

Dimension....................................................... 161

Family-Centered Program Rating Results....................... 168

Parenting Plus Model of Service Delivery............................181

Effective Practices for Intersectoral Program Implementation... 185 


\section{CHAPTER ONE}

Introduction

\section{Overview}

The past decade has seen growing international recognition for efforts targeting the health and well-being of children and their families. In 1989, the United Nations adopted the Convention on the Rights of the Child that defined and promoted the fair and equitable treatment of children worldwide. Foremost within the Convention are clauses associated with the basic needs for nurturance. These include food, shelter, belonging, and love. The Convention also recognizes that equity in education, justice, and special care are inherent civil liberties of the child. Moreover, children were to receive protection from exploitation, war, abuse, and neglect. Thus, the Convention on the Rights of the Child outlines the guiding principles by which nations should conduct themselves concerning the health and welfare of children.

In Canada, social policy initiatives have increasingly reflected our responsibility to improve and promote the well-being of children. Nationally, "Brighter Futures: Canada's Action Plan for Children" (Health and Welfare Canada, 1992), a federal policy document, provides a framework for governmental and non-governmental organizations promoting the interests of children. In Saskatchewan, collaborative efforts between the provincial government, stakeholder advocacy groups, and communities dedicated to the improvement of the health and well-being of Saskatchewan's children resulting in the creation of Saskatchewan's Action Plan for Children (Government of Saskatchewan, 
1993). More recently, a bilateral agreement between the provincial and federal governments created a fund to jointly manage early childhood initiatives in the province to improve the lives of children from birth to five years of age and their families.

There has been a growing demand for intersectoral programs that contribute to the development of a long-term plan for early childhood intervention in the province. A number of community-based initiatives have been implemented by government agencies, non-governmental organizations, and First Nations (see Table 1.1). As grass-roots support for the health and well-being of children and their families in Saskatchewan continues to grow, the demand for reliable, effective, and sustainable early intervention programs has increased.

Table 1.1

Characteristics of Families at Various Levels of Psychosocial Risk

\begin{tabular}{|c|c|c|c|c|}
\hline Characteristics & Low & Moderate & Higher & Highest \\
\hline Lack information & $\mathrm{X}$ & $\mathrm{X}$ & $\bar{X}$ & $\mathrm{X}$ \\
\hline $\begin{array}{l}\text { Lack } \\
\text { support/resources }\end{array}$ & $\mathrm{X}$ & $\mathrm{X}$ & $\mathrm{X}$ & $\mathrm{X}$ \\
\hline Lack skills & & $\mathrm{X}$ & $\mathrm{X}$ & $\bar{X}$ \\
\hline Lack motivation & & & $\bar{X}$ & $\mathrm{X}$ \\
\hline Repeated crisis & & & $\mathrm{X}$ & $\mathrm{X}$ \\
\hline $\begin{array}{l}\text { Child(ren) } \\
\text { manifesting } \\
\text { significant problems }\end{array}$ & & & & $\mathrm{X}$ \\
\hline Services needed & Promotion & Prevention & $\begin{array}{c}\text { Early support/ } \\
\text { intervention }\end{array}$ & \begin{tabular}{c|l} 
Treatment & Interventions \\
Protection & care/custody \\
\end{tabular} \\
\hline \multirow[t]{3}{*}{$\begin{array}{l}\text { Examples of } \\
\text { services }\end{array}$} & \multirow[t]{3}{*}{$\begin{array}{l}\text { Family } \\
\text { literacy } \\
\text { programs }\end{array}$} & $\begin{array}{l}\text { Successful Mothers } \\
\text { Support Program } \\
\text { (volunteer) }\end{array}$ & $\begin{array}{l}\text { Successful Mothers } \\
\text { Support Program } \\
\text { (paraprofessional) }\end{array}$ & $\begin{array}{l}\text { Early Skills } \\
\text { Development } \\
\text { Program }\end{array}$ \\
\hline & & $\begin{array}{l}\text { Teen \& Young } \\
\text { Parent (professional) }\end{array}$ & $\begin{array}{l}\text { Hawaii Healthy Start } \\
\text { (paraprofessional) }\end{array}$ & \multirow[t]{2}{*}{ Treatment Foster Care } \\
\hline & & & $\begin{array}{l}\text { Teen \& Young } \\
\text { Parent (professional) }\end{array}$ & \\
\hline
\end{tabular}

Source: Saskatchewan Health, 1999. 


\section{Purpose}

In 1998, the Pipestone Health District submitted a proposal for health transition funding to the Saskatchewan Health Transition Fund steering committee. The proposal was accepted and funding was granted for the first phase of the pilot program (see Appendix 1). As a result, Parenting Plus, the early childhood development program of the Pipestone Health District has been able to establish a core staff of three that includes a program co-ordinator and two family support workers (FSWs). Their objective is to deliver strength-based home-visiting services to families of newborns in the district who are identified as overburdened.

Parenting Plus is modeled after Hawaii Healthy Start, which is based on Kempe's lay therapy program (Gray, Cutler, Dean, \& Kempe, 1979) and Frailberg's (1980) work in the clinical mental health of infants. Hawaii Healthy Start is a community-oriented intervention program whose primary goal is to prevent child abuse and neglect and in this sense, is directed towards families at high psychosocial risk as illustrated in Table 1.1. Hawaii Healthy Start accomplishes this through the personal development of young parents that includes their ability to use community-based resources to affect positive parenting outcomes. The goals of Parenting Plus (Pipestone Health District, 1998), to be implemented as modeled after Hawaii Healthy Start, are:

1. Systematic screening of high risk families of newborns;

2. Paraprofessional home-visiting program targeted for families living in or with disadvantaged conditions;

3. Informal community support for all families (including those facing significant risks to their health and well-being); and

4. Mechanisms for the co-ordination of services and supports. (p. 1)

By incorporating the above goals into a system of service delivery for early childhood development in the Pipestone Health District, it is believed that Parenting Plus will 
contribute to the improvement of the health, development, and well-being of children and families (Pipestone Health District).

\section{Target Audience}

Parenting Plus targets all families of newborns that reside in the Pipestone Health District who are identified as overburdened. The Pipestone Health District spans the TransCanada Highway from the east side of Regina to the Manitoba border. Parenting Plus uses a two-stage screening, developed for the Hawaii Healthy Start program, to identify eligible families. The purpose behind the development of a two-stage screen is to determine which families are at high levels of psychosocial risk and are eligible for Parenting Plus. In this way, the number of false positives (families identified for services but not in need of them) and false negatives (families not identified for services but who are in need of them) are reduced. If a parent's medical record screen is positive, the program co-ordinator, recommends a family assessment. The assessment identifies families that are overburdened and might benefit from extra support. The FSWs then offer paraprofessional, strength-based home-visiting services to those families.

\section{Evaluation Framework}

In assessing the evaluative needs for Parenting Plus, a series of stakeholder meetings that included the principal investigator were held over a 10-month period leading up to initial data collection. Each of the stakeholders involved had a personal interest in the promotion of early intervention and represented various sectors, including nongovernmental organizations, the University of Saskatchewan, Saskatchewan Health, and 
the Pipestone Health District. Evaluations that include stakeholders and design the evaluation for intended users are epistemologically defined as encompassing pragmatism and popularized as "utilization-focused" evaluations by Patton (1986; 1997a). Furthermore, as Parenting Plus is a pilot program, stakeholders decided that evaluation efforts should be directed towards process as opposed to outcome variables. Stakeholders indicated that information pertaining to the implementation process for Parenting Plus to inform present and subsequent efforts was needed.

\section{Research Questions}

\section{Research Question 1}

As Parenting Plus has been implemented, what is the model of service delivery that has emerged and been operationalized?

\section{Research Question 2}

What characteristics of the implementation process for Parenting Plus have facilitated or hindered the systematic screening of high-risk families of newborns, the provision of paraprofessional home visitations for those families, the development of informal community supports for families, and the mechanisms for the co-ordination of supports and services?

\section{Research Question 3}

What do the participants' experience and what do they understand about the paraprofessional home visitations they receive from Parenting Plus? 


\section{Methods}

The utilization-focused implementation evaluation of Parenting Plus employs both quantitative and qualitative methods for the collection of data. Data collection occurred over a four-month period from September to December 2000. The qualitative methods for data collection included semi-structured depth interviews and focus groups. Twentythree stakeholders with a vested interest in early childhood development in the Pipestone Health District were interviewed. As well, two parent focus groups with six participants each that followed an empowerment evaluation format were conducted. The general inductive approach to qualitative data analysis resulted in the identification of five major dimensions that support a model for understanding the implementation and operationalization of Parenting Plus.

The quantitative methods for data collection included a document review and parent completion of the Family-Centered Program Rating Scale (FamPRS). The document review yielded data pertaining to rates of service delivery and descriptive characteristics of the population screened and assessed. Eleven out of 18 parents participating in the program completed the FamPRS and results were interpreted according to the program manual that enabled an understanding, quantitatively, of the parents' perspective.

\section{Assumptions}

1. It is assumed that the interviewees, the focus group participants, and the respondents to the Family-Centered Program Rating Scale (FamPRS) answered to the best of their ability and honestly, the questions posed in the interviews, the focus groups, and the FamPRS. 
2. It is assumed that the purposeful sampling used to select the participants for the interviews resulted in insight, knowledge, and understanding concerning Parenting Plus.

\section{Limitations}

1. The study is limited in the document review data abstracted and by the interview protocol, the focus group methodology, and the instrumentation used, as these may exclude areas of interest and are not comprehensive in their scope.

2. The study is limited due to the lack of contact with two community-health nurses from health centers in First Nation communities served by Parenting Plus. The result of this failure of communication was that they did not participate in the implementation evaluation.

\section{Delimitations}

1. The study is delimited in that convenience sampling to select participants for focus groups, based on their availability, did not ensure participant representativeness.

2. The study is delimited by the use of empowerment evaluation methods in the focus groups, as the purpose behind their use was not to advocate or ascertain broad social system change. 


\section{Significance}

1. The study is unique in that a utilization-focused framework involving stakeholders resulted in an implementation evaluation.

2. The study is unique in that a mixed methods design using the general inductive approach to qualitative analysis delineated the study's research objectives. 


\section{CHAPTER TWO}

\section{Literature Review}

\section{Overview}

Early intervention has grown rapidly over the past 40 years, and a variety of services have come to be described as early intervention programs. Prior to the 1960 s and early 1970s, very few such programs existed for infants or preschool children who had intellectual disabilities or multiple disabilities or who were at psychosocial risk. The growth in early intervention has occurred because research findings of program evaluations have continued to report positive developmental gains for the child and improved family functioning. Moreover, long-term studies (Barnett, 1993, 1997; Olds \& Kitzman, 1993) of early intervention programs have shown that these highly specialized and comprehensive services are cost effective because they reduce demands on health, education, and justice. Typically, such services are designed to provide primary or secondary intervention to the child and family. However, the services offered are many and varied, making comparisons difficult.

\section{Early Intervention Theoretical Frameworks}

Although there are a multiplicity of early intervention programs that target families and their children, Bronfenbrenner's (1979) work on the ecology of human development has provided the theoretical underpinnings for many of the models of early intervention. This has come about because of the growing realization among early interventionists that, 
to promote healthy development, interventions should be embedded in the ecological context of the child. Briefly, what Bronfenbrenner proposes is a broader approach to research in human development that would focus on the progressive accommodation, throughout the life span, between the emergent human organism and the changing environments in which it lives and grows. Bronfenbrenner takes a systems approach to the ecology of the child and explains how the microsystem, mesosystem, exosystem, and macrosystem interact and influence a child's development. The microsystem refers to the immediate environment of the child that might include, for example, the home or school. The mesosystem refers to the interaction between two microsystems, such as the home and school. The exosystem is an environment in which the child is not involved but, nonetheless, is influenced by, such as the parent's workplace. Finally, the macrosystem is the broader ideology, laws, and customs of the culture in which the child's ecology is rooted. Thus, Bronfenbrenner's theory of human ecology allows us to go beyond the traditional definition of the environment by taking into account the multiple, interconnected systems that influence the child.

Theoretical frameworks of early intervention that are predicated on Bronfenbrenner's work in human ecology include transactional regulation (Sameroff, 1983, 1993; Sameroff \& Chandler, 1975; Sameroff \& Fiese, 1990, 2000a, 2000b), Guralnick's (1997, 1998) early development and risk-factors model, and the resource-based approach to early intervention as developed by Dunst and Trivette (1997). Sameroff and Fiese (2000a), in describing the developmental ecology of early intervention and the theory of transactional regulation, identify three core requirements that are necessary for optimal child development outcomes: 
The first [requirement] is to recognize that child development has multiple contributors at multiple levels of the child's ecology. The second is that at each of these levels, multiple processes are represented in family thought and cultural symbols and are enacted in family interactions and social services. The third requirement is that intervention processes be targeted for a particular child in a particular family in a particular culture. (p. 135)

Given this perspective, transactional regulation theory proposes that we accept the influence of a variety of factors on early childhood development. According to Sameroff and Fiese, no single risk factor in itself is responsible for negative child developmental outcomes. Rather, such results are due to the accumulation of the effects of multiple risk factors over time. The transactional model of early childhood implies that, rather than viewing the developing child within the context of a linear maturational sequence, it is necessary to understand that the individual is embedded in relationships with others who may provide both physiological and psychological nurturance (Sameroff \& Fiese). Unfortunately, however, not all early intervention programs are equipped to deal with the full range of factors that impinge upon successful child developmental outcomes. Therefore, since there is a range of options that early intervention efforts may target, it is vital to outline the opportunities available to families that would have the most significant impact.

Traditionally, research in early childhood development has focused on intervention efforts that target the biological capabilities of the child and are directed towards shaping child competencies (Sameroff \& Fiese, 2000a). However, by focusing only on the child's "phenotype", little effort has been directed toward understanding the child's "environtype", as represented by the cultural, familial, and individual parental codes, in shaping child experiences and competencies. As transactional regulation views the developing child from a systems perspective that follows regulatory principles, 
interventions practiced should target the strengths and weaknesses of the parent-child regulatory system (Sameroff \& Fiese, 2000a, 2000b).

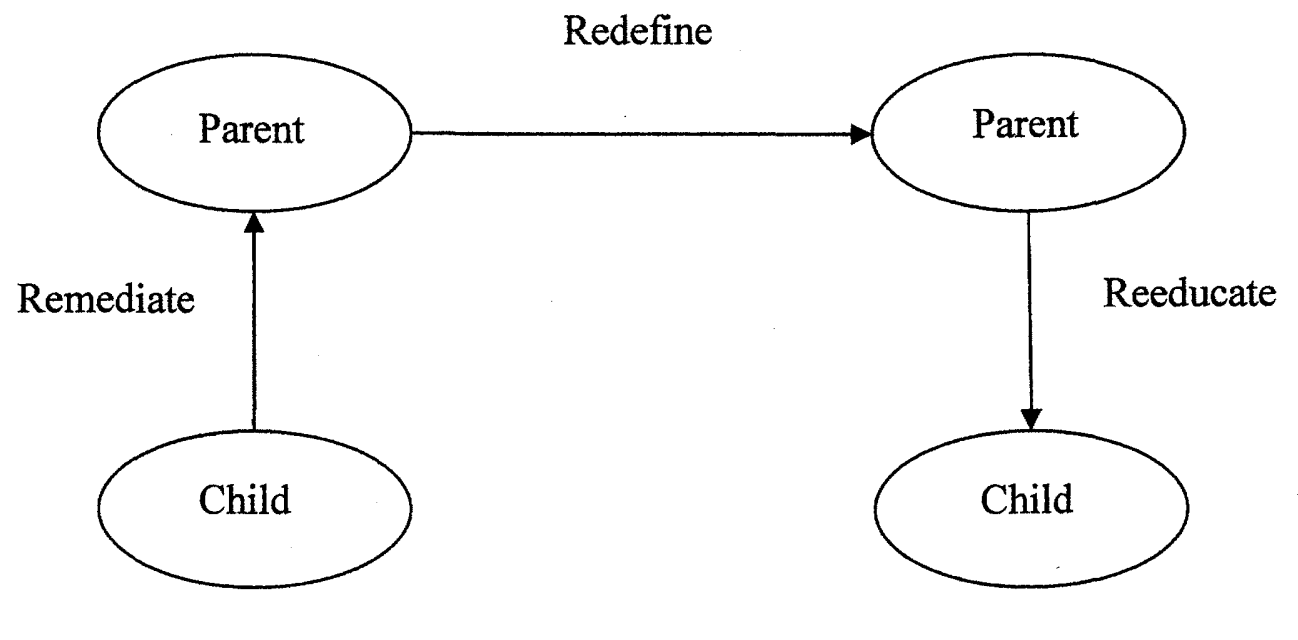

Time 1

Time 2

Figure 2.1. The 3-R's of Early Intervention Within a Transactional Model. Source: Sameroff \& Fiese, 2000a, p. 150.

According to Sameroff and Fiese (2000a), the transactional regulation theory of human ecology has three categories of intervention that can mediate change within a regulatory system (see Figure 2.1). The first category is "remediation" and refers to the behavior of the child towards the parent. The second category of intervention is "redefinition". In redefinition, the parent's interpretation of the child's behavior changes. The third category of intervention is "reeducation". In reeducation, the focus is on changing the behavior of the parent toward the child. In targeting interventions, an analysis of the regulatory systems can provide the most effective avenue for intervention efforts (Sameroff \& Fiese). Moreover, in planning early intervention strategies, Sameroff and Fiese state: 
A basic point that emerges from this analysis is that there will never be a single intervention strategy that will solve all developmental problems. Cost-effectiveness will not be found in the universality of a treatment but in the individuation of programs that are targeted at the relevant nodal points for a specific child in a specific family in a specific social context. ( p.149)

However, transactional regulation has come under criticism because of its narrow focus on changing behavioral patterns (Wolery, 2000). Both Guralnick's $(1997,1998)$ model of early development and risk factors and Dunst and Trivette's (1997) resourcebased model address this shortcoming. What is unique about Guralnick's model is the linkage developed between factors influencing child development and intervention efforts. Guralnick views risk and disability status as potential stressors that can affect family interaction patterns and ultimately child development outcomes. In his model, there are three major components represented as Family Characteristics, Family Patterns, and Potential Stressors (see Figure 2.2).

According to Guralnick (1997), there are also three patterns of family interaction that may act as potential stressors: the quality of parent-child transactions, family orchestrated child experiences, and the health and safety provided by the family. Of the three patterns of family interaction, it appears the quality of parent-child interaction is the most meaningfully affected by early intervention services (Guralnick). This finding is not surprising when it is acknowledged that the family is the primary socialization agent of children from birth to five years. The second factor that can affect child developmental outcomes is found in patterns of family interaction as they relate to the child's relationship with the social and physical environment (Guralnick). Early intervention programs focusing on children with disabilities usually feature this type of intervention service. The third area that parents are responsible for that can affect patterns of family 
interaction and ultimately child developmental outcomes relates to the general health and safety of the family. Because stressors act to effect change in patterns of family interaction, non-optimal patterns are targeted.

\section{Family Characteristics}

Personal characteristics of parents Characteristics not related to child's disability or biological risk status (Social support, marital relationship, financial resources, child temperament)

\section{Family Patterns $\longrightarrow$ Child Development Outcomes}

Quality of parent-child transactions

Family-orchestrated child experiences

Health and safety provided by family

Potential stressors for families created by child disability or biological risk

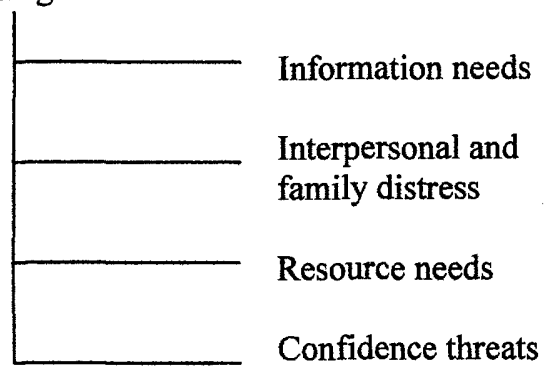

Figure 2.2. Factors Influencing Children's Developmental Outcomes. Source: Guralnick, 1997, p. 7.

As to what constitutes the stressors that affect family risk status, Guralnick (1997) contends that the personal characteristics of the parents are paramount rather than the individual family characteristics or child's biological risk status. According to Guralnick, the family characteristics involved in the development of stressors that can affect family risk status include social support, marital relationship, social and economic status, and the temperament of the child. These factors can influence patterns of family interaction, 
which in turn affect child development. To effect positive change in patterns of family interactions, Guralnick identifies resource supports, social supports, and information and services as being basic to the organization of contemporary services for early intervention efforts (see Figure 2.3).

\begin{tabular}{|c|c|c|}
\hline Stressors & Early Intervention & \\
\hline & Program Components & \\
\hline Resource Supports & Social Supports & Information \& Services \\
\hline $\begin{array}{c}\text { Awareness of and access to, } \\
\text { and primary coordination } \\
\text { of services }\end{array}$ & $\begin{array}{l}\text { Parent to parent groups } \\
\text { Family counseling }\end{array}$ & $\begin{array}{l}\text { Formal intervention program } \\
\text { (home/center based) }\end{array}$ \\
\hline $\begin{array}{l}\text { Supplemental supports } \\
\text { (financial assistance, respite care) }\end{array}$ & $\begin{array}{l}\text { Mobilize family/friend/ } \\
\text { community networks }\end{array}$ & $\begin{array}{l}\text { Parent-professional relationships } \\
\text { (health and safety issues, } \\
\text { anticipatory guidance and } \\
\text { problem solving) }\end{array}$ \\
\hline & & Individual Therapies \\
\hline
\end{tabular}

Figure 2.3. Components of Early Intervention.

Source: Guralnick, 1997, p. 9

Guralnick (1997) contends that by providing an integrated system of service delivery for early intervention, the stressors that place families at-risk are reduced. Of the three categories of services he identifies, Resource Supports is one of the most important for families at psychosocial risk. As many of the families served by early intervention services are poor, unemployed, and single parents, the challenges they face are multifaceted. For these families, even the most adept early interventionists have widespread difficulties in accessing and co-ordinating access to health, education, and social services (Guralnick). Moreover, with the rise of parent advocacy and support organizations, early interventionists have increasingly relied on these groups to assist 
parents and their families in developing social supports to reduce the stressors that inhibit healthy child development. Nevertheless, these services in themselves are not always enough. Therefore, in order to affect positive change in family interactions, many early intervention programs include a home-or center-based component. Although this is one of the more significant and common features of early intervention services, it is also one of the most expensive for community-based programs.

The third theoretical framework that has come to characterize early intervention is the resource-based model as conceptualized by Dunst and Trivette (1997). Like the ecology of human development as developed by Bronfenbrenner (1979), the resource-based model assumes that both the child and the family are embedded within a number of ecological systems. However, what differentiates the model is that it is based on developing partnerships and empowering families, as opposed to a professional decisionmaking process that prescribes treatment and usurps the parent's role (Wolery, 2000).

The resource-based model is viewed as a strength-based approach that recognizes that the recipient's perception of the intervention is highly related to its effectiveness (Trivette $\&$ Dunst, 2000). In the resource-based model (Dunst \& Trivette, 1997), the three essential components identified include Sources of Support, Community Resource Mapping, and Building Community Capacity. What is unique about the resource-based model is that it draws on existing programs in the community to facilitate and aid in intervention services. Moreover, it expands the conceptualization of community resources for support by including not only an individual's social network but also associated groups, community programs and professions, and specialized services (Trivette, Dunst, \& Deal, 1997) (see Figure 2.4). Although the resource-based model has existed for a number of 
years, it has not been adopted in its entirety by early intervention programs. Nevertheless, the strength of this model lies in its ability to aid families in recognizing and prioritizing their needs on the basis of existing resources and sources of support available in the community.

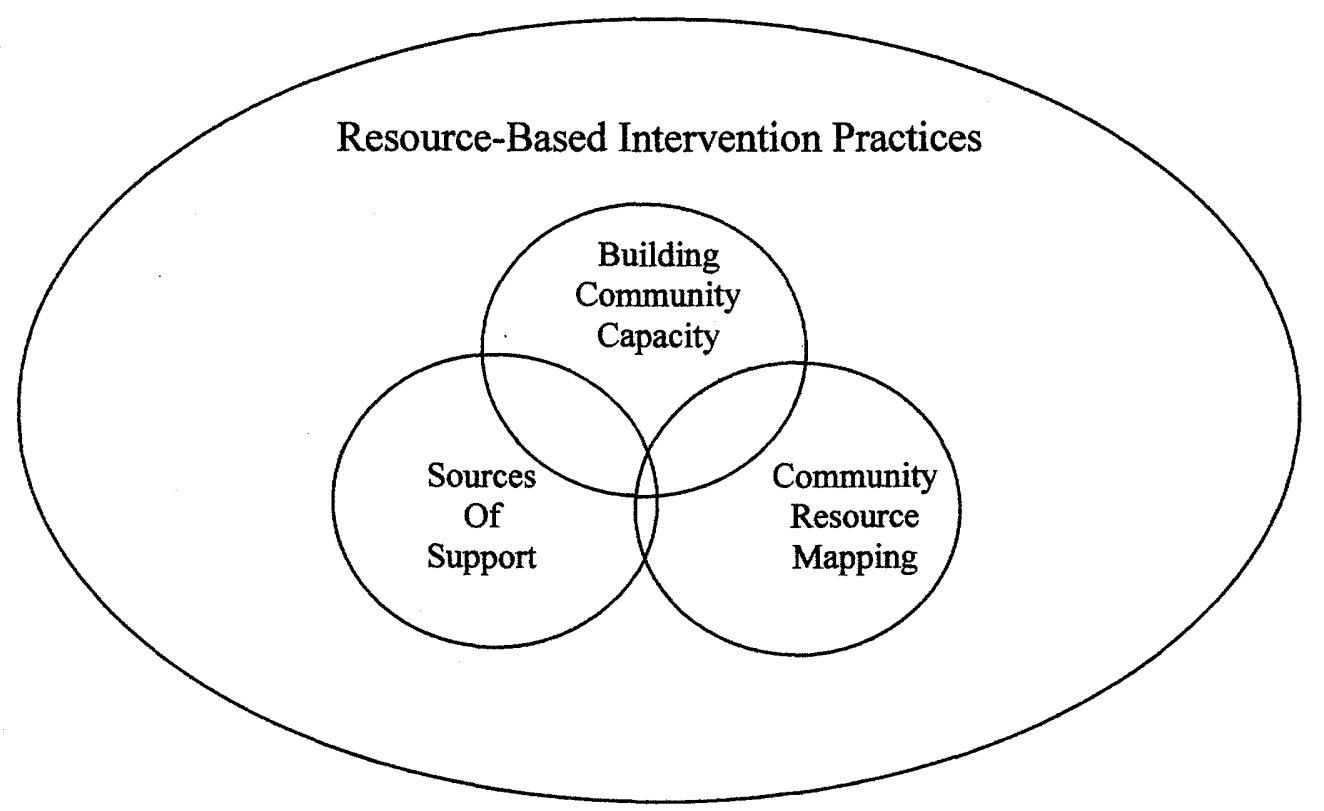

Figure 2.4. Resource-Based Model of Early Intervention.

Source: Trivette, Dunst, \& Deal, 1997, p. 84.

\section{Home Visiting Programs}

Traditionally, descriptions of early intervention services have been dichotomized into home-based or center-based programs. Within this classification scheme, there are a number of early intervention efforts that vary both theoretically and pragmatically in program delivery. Thus, the diversity of home-visiting programs is not only predicated on the theoretical assumptions of early intervention models developed and directed towards the special needs of the target population; it is also found in the frequency and duration of 
the home visits, the profession of the service provider (educator, paraprofessional, social worker, nurse, etc.), and the types of interventions practiced (Hauser-Cram, 1990).

General findings in the research literature argue that home visitations, either alone or in combination with a center-based approach, have demonstrated consistent results in improving maternal and child health, providing social support, and reducing child abuse and neglect. Economic evaluations of home-visiting programs have largely included costeffectiveness analysis, cost benefit analysis, and cost utility analysis. Unfortunately, most studies conducted on home visitation effectiveness have not included an economic evaluation. Those that do vary not only in the goals, methods, and target population, but also in the valuation applied to selected benefits. Nevertheless, in a review of the literature, it was found that in the short (Barnett \& Escobar, 1990; Olds, Henderson, Tatelbaum, \& Chamberlain, 1988; Olds \& Kitzman, 1993) and the long term (Barnett, 1993, 1997; Olds \& Kitzman), home visitations are reported as providing positive economic benefits and being potentially economically viable.

It is clear from the discussion thus far that infant health, development, and behavior are complex and sophisticated expressions that "have many definitions and implications for [early] intervention strategies" (Ramey \& Ramey, 1993, p. 130). To alleviate this ambiguity, Ramey and Ramey argue that it is necessary to view health and development within a conceptual framework embedded in the resources of the families, communities, and children. According to Ramey and Ramey, this framework recognizes that the developmental and ecological contexts of young children are dynamic and, thus, is congruent with the theories of Bronfenbrenner (1979) and Sameroff, and Fiese (2000a, 2000b). In conducting a content analysis of the literature on the effect of home-visiting 
programs regarding the health and development of young children, Ramey and Ramey conceptualized eight distinct yet interdependent developmental domains of functioning:

1. Survival-housing, income, food, clothing, safety, and transportation;

2. Values and goals-to succeed as family, to do well in school and work, and to be part of the community;

3. Sense of security-physical, emotional, and financial;

4. Health-physical and mental;

5. Social interaction-with family, with peers, and with community;

6. Self-esteem, personal competence, academic cognitive competence, and social competence;

7. Communication-listening skills, speaking skills, reading skills, and writing skills; and

8. Intellectual skills-everyday living skills, social-emotional skills, and academic and work skills. (p. 131)

It is important to realize that home-visiting programs place different and selective emphasis on the various domains of functioning. Accordingly, Ramey and Ramey abstracted four factors that influence a home-visiting program's decision on which domains to emphasize and which to disregard. The factors that influence the delivery of early intervention home-visiting services include the anticipated and personal needs of the population, the orientation and professional expertise incorporated in program design and implementation, resource availability, and the program's philosophical orientation.

In a review of the experimental literature regarding the effectiveness of home-visiting programs that incorporated randomized trials, Olds and Kitzman (1993) examined 31 home-visiting programs. The decision to focus on randomized trials as a means to ascertain program effectiveness was chosen by Olds and Kitzman because studies that incorporated this type of research design were thought to be more methodologically sound. The review supports the contention that early intervention home-visitation programs are diverse in nature due to differences in the target population's needs and the type of programming offered. In summarizing the categories of program content delivered, Olds and Kitzman found that the following themes began to emerge: 
1. Improvement of parental behavior through parent education or health related maternal behavior/cognitive stimulation of the child.

2. Provision of informal or formal social support through integrating service delivery and home visitor's emotional or instrumental support.

3. Improvement of maternal life course development through education, finding work, and planning pregnancies. (p. 55)

In conducting their research, Olds and Kitzman (1993) discovered some interesting trends in the characteristics of the various programs. Generally, it is found that prenatal programs tend to reduce psychosocial stress through the enhancement of informal and formal supports. Furthermore, only three of the seven prenatal programs reviewed consider parent behavior, specifically as it relates to health education, significant enough to warrant intervention services. Those programs geared towards serving parents of preterm and low birth weight babies tend to focus on improving both parental behavior and informal and formal social supports. The parenting behaviors considered relate to parent education and parental cognitive stimulation of the child. Informal and formal supports are obtained through counseling of the mother, family, and friends in the program. The more generalized and comprehensive programs, though, as based on the number of component characteristics found, are those for low-income families or families at risk for child maltreatment. Different theoretical approaches are taken to guide these interventions due to the complex and multifaceted nature of the problems. This lead Barnett (1997) to conclude:

Transient interventions targeting narrow areas may be effective for acutely maltreating families that have experienced short-lived challenges. However, brief treatments are unlikely to prevent or ameliorate child abuse and neglect among families with chronic and severe potentiating factors. In such instances, interventions require comprehensive programs providing long-term services. (p. 149) 
Although the review provided by Olds and Kitzman is not inclusive of all types of early intervention programs and program characteristics, it does provide a thumbnail sketch of what constitutes early intervention home visitations.

To delineate the processes involved in the provision of home visitations, Kitzman, Olds, Cole, and Yoos (1997) conducted a qualitative study of the challenges experienced by home visitors for a program implementation evaluation. Kitzman, Olds, et al.'s research drew upon the Memphis randomized trial of prenatal and early childhood home visitation conducted by Olds $(1987 ; 1992)$. Although, Blair and Ramey (1997) identify the need to determine how family characteristics and program implementation interact to optimize services as being integral to successful program adaptation and stabilization, there is a paucity of research on how successful adaptations affect the delivery of a program. In Kitzman, Olds, et al.'s study, each of 17 nurses was asked to provide detailed monthly audio-taped reports of their work with two families. Although, the nurses could discontinue their service at any time, the study reported that the average length of participation by the nurses was 17.3 months. The research team read and analyzed verbatim transcripts from the audiotapes. As a testament to the comprehensiveness of the data collected, the study reports that the text of the verbatim transcripts provided by the nurses for the families was up to 100 pages long in some cases. Kitzman, Olds, et al. describe the process of data analysis as follows:

Data analysis consisted of first identifying from the narrative reports the themes that best characterized the interactions between nurses and the families over time. Those themes were then placed in broader cultural and social perspectives through exploration of relevant literature, and consultation, with experts, nurse home visitors in the program, and other informants. Insights from these various resources were integrated into subsequent readings of the narratives in a circular process moving from description to interpretation to initial understandings. (p. 98) 
Thematic analysis of the narrative reports by the research team identified the obstacles faced by the nurses in the delivery of the home-visitation program. The results reveal that the nurses were confronted with nine main challenges, many of which were interdependent. Kitzman, Olds, et al. highlight the challenges, although the researchers recognize their mutually dependent nature:

1. Gaining and maintaining access to the families;

2. Facing limits posed by the environment;

3. Identifying and engaging all relevant players;

4. Delivering the protocol elements;

5. Balancing nurse responsibility and client responsibility;

6. Balancing maternal life-course goals with child and family needs;

7. Maintaining a balance between a future orientation and a present orientation;

8. Managing in the face of cultural complexities;

9. Waiting for readiness of mothers to change. (pp. 98-106).

Although the home visits occurred in a large North American city, the first challenge, gaining and maintaining access, proved to be one of the most significant faced by nurses who participated in the study. Unlike center-based programs where the client is served within an institution representative of professional practice, home visitations occur in the context of the client. The frequency, intensity, and agenda followed during home-visiting are at the discretion of the parent. Of the many appointments that were not kept by clients, nurses believed that some were missed intentionally. If the missed appointment was unintentional, nurses believed it was the result of the multiple demands upon parents' time. If the failure to meet was intentional, it was attributed to a lack of motivation or the challenging nature of some of the previous home visits. For the nurses delivering the home-visiting program, one of their overriding goals was to maintain access and contact with the families to ensure continuity of service and quality of care. Often the nurses were in a situation where, in their best clinical judgment, intervention due to problematic 
behaviors was warranted. When this occurred, the nurses usually tested the boundaries in terms of the parents' ability to communicate in an open or closed manner about the issue in question. Decisions to be confrontational were not characteristic of specific nurses but more a function of the type of problem encountered during the home visitations. Thus, according to Kitzman, Olds, et al.:

Deciding when to confront seemed to depend on the nature of the conflict rather than the characteristics of the nurse. The same nurse would back off from confronting a certain problem and yet confronted others when she felt a situation posed a risk to either or both the mother and child. The dance of confronting and backing off involved selecting and titrating the challenges, providing as many small challenges for growth as the family could manage successfully at any point in time, without providing so many challenges that family members either became overwhelmed or failed to meet them. ( p. 99)

Not only did the nurses find it difficult to access and maintain contact with the families, they also found that the physical environment imposed limitations (Kitzman, Olds, et al., 1997). Some of the nurses found that families objected to discussing sensitive issues, while other nurses indicated that the highly impoverished conditions faced by many of the families made it difficult for them to attain some of the goals set out by the program. For example, several of the nurses provided the families with recommendations to create a more safe and secure home environment. Unfortunately, many of the parents, out of fear of harassment from their proprietors or due to a sense of learned helplessness and the futility of making recommendations, took little or no action to improve their living conditions. Nonetheless, the nurses were impressed by the resiliency of the families in the face of such adverse conditions (Kitzman, Olds, et al.).

The third major challenge articulated by the nurses related to identifying and engaging all the relevant players involved in the home visitations (Kitzman, Olds, et al., 1997). The intervention provided by the nurses involved identifying the family system 
and subsystems and then prescribing intervention activities for the mother-child dyad, incorporating other family members when appropriate. This could mean focusing on the father figure and the grandmother. Some of the difficulties faced by the home visitors were due to shifting patterns of familial composition that made it difficult to decide on whom to focus the intervention. Furthermore, the degree to which fathers were involved, the multiple father figures in the life of the child over time, and the diverse roles that grandmothers and extra familial individuals played, all contributed to challenges faced by the nurses. To overcome these obstacles, the nurses did their utmost to ensure that the optimal development of the mother and child was promoted, without threatening or jeopardizing the extra-familial bonds. This was especially difficult for some of the nurses when they encountered other family members who were themselves in considerable distress but were not the focus of the intervention. Often the dilemma arose as to where to draw the line in rendering services. Moreover, the supports and services offered the families were sometimes in direct conflict with the role expectations of the families as prescribed by extended family members. In this respect, Kitzman, Olds, et al. state:

Working within the family as the context for care delivery proved to be one of the greatest challenges for the nurses. While the extended family was a support system that committed valuable family resources to the young mother, there was a direct price to be paid. Reciprocity, obligation, and connectedness often were in direct conflict with the young mother's individuation and engagement in activities fostered by the program. Many mothers, for example, had multiple responsibilities for care of their own child, other children within the extended family, as well as aged family members. For them, it was more difficult to simultaneously improve the care of their own child and complete their schooling or find work. (p.101)

When it came to delivering the program protocol, nurses faced obstacles owing to the diversity and variability of the families' needs. For some families, finding shelter, other than temporary residences and basic subsistence, tended to dominate. In contrast, families 
with resources upon which they could draw were more upwardly mobile and, therefore, required the co-ordination of ancillary services to prepare for careers and later life (Kitzman, Olds, et al., 1997). Another impediment was trying to deliver a program protocol at odds with the family's needs. For example, one aspect of the program related to solving problems faced by families that included adult relationships and material resources. The nurses felt that by focusing only on these problems and their remediation, other relevant program concerns, such as child welfare, were overlooked. Less time and opportunity were available to help parents anticipate and plan strategies for addressing problems concerning their children's health and development (Kitzman, Olds, et al).

Another challenge the nurses faced in delivering the program protocol related to limited literacy on the part of some of the parents receiving services and the variability of acceptance by parents when considering the infant-parent activities advocated by the program. This required the nurses to modify course materials and either make them more understandable or, for those who were able to go beyond basic materials, provide auxiliary resources. Consequently, to address the limited engagement of some of the parents in the parent-infant activities, nurses needed to modify and tailor projects to the lifestyles and needs of individual parents and families.

The fifth major challenge faced by nurses related to balancing nurse and client responsibility. Kitzman, Olds, et al. (1997) found nurses continually confronted with professional and moral responsibilities in their decisions to help or advocate for families. For example, nurses often faced the decision of whether to allow families to help themselves, although this might mean failure on the part of the family, or to assist the families to complete the necessary work required to meet their needs. Thus, when the 
nurses faced the challenge of completing the tasks on behalf of the family, they usually did so if to do otherwise would jeopardize the family's well-being and place them at greater risk (Kitzman, Olds, et al.).

The sixth challenge faced by nurses related to the balancing of maternal life course goals with child and family needs (Kitzman, Olds, et al., 1997). Often nurses had to decide whether the mother's pursuit of her own goals and interests outweighed, for example, her care-giving responsibilities. This was especially apparent when issues related to employment and education for young mothers occurred. Nurses had to determine if the benefits associated with employment and education outweighed the opportunity to become fully engaged in the raising of the infant. Further complications arose in cases where the young mother worked to support other family members, as well. The nurses felt that, by supporting the mother's decision, they were in direct conflict with program protocol that promoted the health and-development of the infant (Kitzman, Olds, et al.).

The seventh major challenge was to maintain a balance between future and present orientation (Kitzman, Olds, et al., 1997). Consistent with the protocol, the nurses promoted future-oriented goals based on the following premises:

1. Contraceptive practices and planned pregnancy offer the woman control over her life;

2. Education leads to future employment and economic self-sufficiency; and

3. Sensitive parent-child interaction promotes children's later behavioral adjustment and school achievement. (p.104)

The nurses also reported that the promotion of future-oriented goals was at times in conflict with the parents' perception of the future and how they assessed their basic survival needs. At other times, the nurses recognized that the overwhelming subsistence 
needs of some of the clients required addressing first. This was necessary because to do otherwise would impede the attainment of future-directed goals. Nevertheless, when nurses opted for actively pursuing the survival needs of the parents, to the neglect of the protocol's future-goal orientation, the effect of the future development of the child became a concern (Kitzman, Olds, et al.).

The eighth challenge faced by the nurses was respecting and understanding the cultural context of the families and its effect on childrearing. The study reported nurses hesitated to implement or advocate for the adoption of program practices related to care giving and life style as suggested by the protocol if they believed the practices were not congruent with the cultural context of the family (Kitzman, Olds, et al., 1997). The difficulty that faced many nurses was deciding when a particular behavior had a cultural foundation and might therefore serve a useful function within the family and when it did not.

The final challenge faced by the nurses (Kitzman, Olds, et al., 1997) was in regard to mothers' readiness to change. In part, this is a function of a program that tried to unify goal-oriented activities with the day-to-day survival of the parents and families. The nurses were concerned that some of the developmental needs of the children were being unmet, which could lead to negative lasting effects on the children. Thus, nurses tried to optimize their visits and create a balance between the child's developmental needs and the long-term opportunities for parents (Kitzman, Olds, et al.). According to the researchers, this presented as a major challenge to the delivery of the program protocol.

In discussing the findings of the research and its subsequent implications for homevisiting programs, the authors (Kitzman, Olds, et al., 1997) found, first of all, that many 
of the challenges faced by the nurses were expected, given the multidimensional nature of program delivery. Furthermore, the nurses, by acknowledging and responding to the challenges faced by the families and to themselves in the delivery of the program protocol, enhanced the quality of intervention services offered. According to Kitzman, Olds et al.:

Families responded to the visitors, in part, because the visitors understood and acknowledged the families' difficulties in responding to the content of the program, given their needs and contexts. The tension produced by competing agendas precludes concentration on one aspect of the individual and family development to the detriment of another. The comprehensive nature of the program meant that nurses were sensitive to most of the critical forces influencing women's efforts to achieve economic self-sufficiency and competent parenthood. (p. 107)

Moreover, success was due to the nurses planning and continuous assessment of the family and the effects that program implementation had on the parents and children. This resulted in considerable effort on the part of the nurses in retaining those families at greatest risk. It is Kitzman, Olds, et al.'s contention that such a practice attracts and retains families who otherwise might be overlooked because of perceived lack of interest or being difficult to locate.

The second major finding and its implication for future home-visiting programs related to the challenging but essential task of working with entire family networks (Kitzman, Olds, et al., 1997). Consequently, the nurses involved in home visitations had to demonstrate a certain degree of flexibility in how they chose to work with the parents, other family members, and children. At times, the nurses worked on an individual basis with the parents toward meeting their goals or in conjunction with other family members or individuals. Because intervention programs have the potential to change family dynamics, Kitzman, Olds, et al. stress that the incorporation of other family members 
requires practitioners to evaluate how these changing dynamics affect the family in order to determine the program's effectiveness.

The third major finding reported by Kitzman, Olds, et al. (1997) related to the competing agendas of maternal well-being and care giving:

The nurses described situations where the demands in one area of mothers' lives were so high that the mothers failed to make satisfactory progress in others. It sometimes was difficult within the course of the program to determine how women's personal goals, goals for her child, and family responsibilities could be balanced. (p. 108)

In part, this difficulty is inherent in any program that attempts to affect multiple domains and the future health and well-being of the child and mother. This same challenge might not be present if the program had a single theoretical basis or if it only operated in the short term. Nevertheless, creating a safe and secure environment in the home for the raising of infants and children requires a program to build on parental efficacies. It is important to remember that parental competency is not only related to child rearing concerns but also the broader area of life skills as influenced by the social and physical environment (Kitzman, Olds, et al.). Thus, Kitzman, Olds, et al.'s research provides qualitative evidence on how effective program processes can be adapted to suit the characteristics and needs of the client population they serve.

Returning to the conceptual framework for the health and development of young children in relation to home-visiting programs as advocated by Ramey and Ramey (1993), it is important to remember that young children's health and development is complex and embedded in the context of the individual, family, and community. The multidimensional domains of heath and development in young children highlight the importance of early intervention being sensitive to these conditions (Bronfenbrenner, 1979; Dunst \& Trivette, 1997; Guralnick, 1997; Ramey \& Ramey; Sameroff \& Fiese, 
2000a, 2000b). Kitzman, Olds, et al. (1997) also report that it is clinically challenging to integrate all program goals into a single program. In a qualitative case study conducted by Kitzman, Yoos, Cole, Korfmacher, and Hanks (1997), it was hypothesized that the integration and simultaneous addressing of program goals intensifies the influence on program outcomes.

To address this research hypothesis, Kitzman, Yoos, et al. (1997) conducted a study in which a single case was selected from the Memphis New Mothers Study for further investigation. The Memphis New Mothers Study had previously been reported in the research literature by Olds, Henderson, Tatelbaum, and Chamberlain (1986) and Olds, Henderson, Chamberlain, and Tatelbaum (1986), who had conducted a randomized trial for those mothers who participated in the home-visitation program. Insights gained from Kitzman, Yoos, et al. relate to how theoretical program protocol can be integrated into the content of the program. In conducting the case study, the researchers drew upon multiple sources of data from the family and the young woman involved in the intervention. Data collected for the study occurred from the period commencing in the young woman's 10th week of pregnancy until the infant was one year of age. Structured and semi-structured interviews with the infant's mother and grandmother, along with nursing records and developmental assessment of the child, comprised the data collection. The purpose of the research study was to demonstrate how home visitations, which are required to operationalize abstract theory regarding program goals, can be individualized so as to meet the needs of the participants that the program intends to serve. 
In discussing their research as it relates to program theory and implementation, Kitzman, Yoos, et al. (1997) conclude:

A preventive intervention for families increases its chances for success when it is grounded in well-articulated and valid theories of human behavior and development. The current case illustrates how the nurse's simultaneous attention to Tamica's sense of self-efficacy, to Tamica's relationship with her child and with her mother, and to the larger family and social contexts in which this family was developing, guided the nurse in the development of a sensible treatment plan. (p. 40)

Although the findings are specific to a single case study and therefore are not generalizable, they underscore the impact that home visitations can have on program participants when an effort is made to tailor program theory, goals, structures, and processes to the individual needs of the clients.

In Britt's (1998) study, where the case was the program itself, researchers sought to explore how changes in the context of a program effect service delivery and how such changes affect families. The home-based preschool program called the Home Instructional Program for Preschool Youngsters (HIPPY) targeted at-risk parents of four and five year olds. The program conducted in a midwestern city had a staff that originally consisted of a program co-ordinator and two paraprofessionals. After one year of service delivery, additional funding enabled the acquisition of supplementary resources to augment the delivery of the program. Thus, the context of service delivery changed over the two years of the study (Britt). The research team then sought to determine how this change affected the ability of the program to serve parents and families. To ascertain the meaning of the changing context of service delivery to the participants, one and one-half hour, semi-structured interviews with 14 of the 80 families took place. The researchers also conducted interviews of one hour in length with the program staff and drew upon field notes, program documentation, and paraprofessional assessments to enhance the 
validity of the data collected. Not surprisingly, Britt found that in the first year of the program, when resources were constrained, it was difficult for program staff to accommodate the mandate of the program. As a result, much of their efforts were directed towards determining how practical interventions would be, considering the constellation of family characteristics the paraprofessionals faced. Paraprofessionals encountered a situation where they had to make decisions regarding the viability of intervention based on the receptiveness and abilities of the families involved. Britt, in presenting his findings for discussion states:

With the constrained resources available during the first year of the program, the need to reach out to families and make a difference in their lives had practical limits on involvement that were reinforced by moral decisions regarding how 'workable' the families were. Consequently, efforts involved working through the program to increase the capacities of parents to deal with problems or to lessen the potentially negative impact of problems on program involvement. With the addition of a family support specialist in Year Two, the tension generated by the dilemma between the relatively narrow focus of the HIPPY program was greatly reduced. The staff members were able to reach out to families with greater confidence and make a difference in their lives by attacking the problems rather than symptoms. (p. 114)

What is learned from this experience is that inadequate funding compromises service delivery and forces programs into reactive or crisis interventions as opposed to proactive, strength-based models of helping. This is especially true for new or evolving programs that are trying to establish service delivery with limited resources.

The three qualitative studies referred to have increased our knowledge about process variables including challenges presented in the delivery of early intervention services, the integration of program theory and protocol with family characteristics and needs, and how a change in context can significantly impact on the quality of early intervention (Blair \& Ramey, 1997; Britt, 1998; Kitzman, Yoos, et al., 1997; Kitzman, Olds et al., 1997; Ramey \& Ramey, 1993). Although these findings expand the knowledge base of 
home-visitation programs, none of the qualitative studies referred to target the prevention of child abuse and neglect. This is significant because home visitations that attempt to prevent or ameliorate these problems usually tend to be more comprehensive. Moreover, programs that attempt to prevent child maltreatment usually provide long-term services owing to the chronic and potentiating influence of psychosocial risk factors facing families (Barnett, 1997; Olds \& Kitzman, 1993; Werner \& Smith, 1992). To address this shortcoming, the review of the literature will focus on those early intervention programs designed to prevent child abuse and neglect.

\section{Child Abuse and Neglect}

Child abuse and neglect are widely recognized as urgent public health concerns. In the United States, it is reported that over one million children annually are abused (Ekenrode, Ganzel, Henderson, Smith, Olds, Powers, Cole, Kitzman, \& Sidora, 2000). In a recent Canadian study, the incidence rate of child maltreatment investigations is reported at 21.5 per 1,000 children (Tromce $\&$ Wolfe, 2001). Of these investigations, $45 \%$ were substantiated, $22 \%$ were suspected, and $7.09 \%$ were unsubstantiated. According to Tromce and Wolfe, an examination of the primary reasons for the investigation of child maltreatment in Canada reveal the following:

Child neglect was the most common reason for the investigation $(40 \%$ of all investigations), followed by physical abuse (31\%), emotional maltreatment (19\%), and sexual abuse (10\%). (p. 11)

Moreover, of the four types of child maltreatment, emotional maltreatment had the highest substantiation rate at $54 \%$, followed by neglect at $43 \%$, sexual abuse at $38 \%$, and physical abuse at $34 \%$. Further figures for substantiated child maltreatment cases reveal 
that more than $43 \%$ occurred over a period in excess of six months and that $56 \%$ of these were cases of emotional maltreatment. Not surprisingly, when the substantiated incidence rates for single occurrences are examined, the figures reveal that $46 \%$ of physical abuse cases and $29 \%$ of sexual abuse cases involve single incidents. This finding is expected given that both physical and sexual abuse are more readily identifiable acts that can be substantiated.

Unfortunately, the effects of child abuse and neglect are long lasting and can cause the most severe damage to the healthy psychological development of the child (Ekenrode et al., 2000). Follow-up studies of first-time juvenile offenders and inmates in federal penitentiaries indicate that the vast majority were abused as children. Evidence indicates that infants are more likely to be abused or neglected than any other age group. According to Barnett (1997):

Infants and toddlers may be at greater risk for maltreatment because they are not capable of meeting their own needs and therefore place high demands on their caregivers' resources. Young children also lack the capacity to escape from or placate an imminently abusive parent. These vulnerabilities may be reasons why infants are more likely to be maltreated than any other age group and why abuse remains the leading cause of death. (p. 147)

If infants are the most vulnerable for child abuse and neglect, it is not surprising that early intervention home visitations are viewed as viable. Moreover, the wide promotion of home visitations as a promising approach to preventing health and development problems in children has aided their popularization as an intervention for maltreating parents (Ekenrode et al.). However, few studies have examined conditions under which such programs meet their goals (Duggan, McFarlane, Windham, Rohde, Salkever, et al., 1999; Duggan, Windham, McFarlane, Fuddy, Rhode, et al., 2000; Ekenrode et al.). Furthermore, empirical research on the continued effect of home visitations on 
maltreating parents once the intervention is withdrawn is scant. Nevertheless, legislators and policy makers view home visitations as an effective means of reducing child abuse and neglect.

In order to understand the complexities surrounding psychosocial risk factors and the etiology of child abuse and neglect, a brief review of the relevant research is required. Although maltreatment occurs in all socioeconomic groups, clinical reports, surveys, and official statistics consistently find that it is most likely to occur among the poor or disadvantaged (Wolfe, 1991) with over $44 \%$ of substantiated cases of child maltreatment involving single parent households (Trocme \& Wolfe, 2001). The child's natural parents, for the most part, are the perpetrators of the abuse. However, even though the majority of abusive parents are in the low socioeconomic strata, more than $95 \%$ from this group do not abuse. A mitigating factor that exacerbates the effects of poverty and the potential to abuse or neglect children is some form of parental history of maltreatment (Barnett, 1997). Factors relating to parental personality and skills also are associated with the chronic occurrence of child abuse and neglect. Of these factors, those which affect parental behavior and attitude are the most receptive to change. Unfortunately, the causes of poverty and unemployment remain deeply entrenched. Abusive parents often begin their families at a younger age than the average, with many still in their teens with the birth of their first child. This suggests that younger parents may be less prepared to raise children, given the teenager's maturity level and limited ability to develop social supports and economic security. It is not surprising then that many early intervention home visitations that attempt to prevent child abuse and neglect target poor, single parents.

When reviewing the types of programs that are developed to prevent child abuse and 
neglect, Gray and Halpren (1988) found tremendous heterogeneity among intervention efforts. Like other home-visiting programs, those that target child abuse and neglect vary in their program characteristics, making comparisons across programs difficult. However, in reviewing the literature, certain trends began to emerge. Specifically, programs that focused on changing parental behavior through home visitations were the most effective (Gray \& Halpren). This is an important finding because poor parenting skills are identified as one of the predisposing factors that place parents at greater risk for maltreatment of children (Barnett, 1997). As well, when the research results of quantitative meta-analysis of child abuse and neglect programs is consulted, some interesting trends begin to emerge. Specifically, in Guterman's (1999) investigation that compared population-based and screening-based home-visitation programs targeting child abuse and neglect he reported:

...population-based enrollment strategies appear favorable to screening based ones in early home-visitations programs seeking to prevent child abuse and neglect. It may be that psychosocial risk screens serve to enroll higher proportions of families for which early home visitations are less likely to leverage change and exacerbate a mismatch between early home-visitation service aims and family needs. (p. 863)

What is of interest in Guterman's study was the finding that home-visitation interventions that use screening-based enrollment strategies do not demonstrate robust outcomes. Thus, it is misguided in Guterman's opinion to continue to validate screening-based programs on the argument that by targeting resources in such a manner that those most in need will receive services. Rather, if robust outcomes are desired, then it would be more appropriate to employ demographically-based enrollment criteria in high-risk communities (Guterman). Early intervention home-visitation programs designed to prevent child abuse and neglect might produce more favorable outcomes if they were 
offered on a universal basis in those communities whose demographics are predominately characterized by single parent teen families living in poverty (Guterman).

However, not all screening-based programs are the same, and some have reported positive outcomes. Of those programs that offer home visitations to affect changes in parental behavior, Hawaii Healthy Start is one that has been extensively reviewed and subject to academic scrutiny in the research literature. Furthermore, as Hawaii Healthy Start is the model for Parenting Plus, a review of the research literature as it pertains to the Hawaii Healthy Start program is warranted.

\section{Hawaii Healthy Start}

Hawaii Healthy Start is a community-based intervention program designed to prevent child abuse and neglect. In Hawaii Healthy Start, this is accomplished through the personal development of young parents that includes their ability to utilize communitybased resources to effect positive parenting outcomes and the healthy development of young children. Hawaii Healthy Start owes its genesis to Kempe's lay therapy program, as reported by Gray et al. (1979) and Frailberg's (1980) work in the clinical mental health of infants. Hawaii Healthy Start began in 1975 as a single site on Oahu, where over $80 \%$ of the Hawaiian population resides. Since then, it has spread to over fourteen sites in the United States. In Hawaii, with a budget exceeding $\$ 6$ million, over 8,000 families have been screened for risk factors, with 2,500 enrolled in home-visitation services. In Canada, the program has been implemented at several sites including British Columbia, Alberta, and Manitoba's Baby First program.

In order to achieve the objectives of the Hawaii Healthy Start program, a model of 
service delivery was established. The model is comprised of four components that include early identification of families at-risk, community-based home visiting services, linkage to primary health care services, and referral and co-ordination with community services (Hawaii Department of Health, Maternal and Child Health Branch, 1994a). The first component of the model calls for hospital-based screening and assessment of newborns and their families. Once a family is determined to be at psychosocial risk, home-visitation services by paraprofessionals are provided.

The second component is the home-visitation services that include enhancing parentchild interactions and bonding, needs identification and referral to appropriate services, parent skill building, case co-ordination, and maintenance of a client tracking data system to document client risk level and program impact (Hawaii Department of Health, Maternal and Child Health Branch, 1994a, 1994b).

- The third component of the Hawaii Healthy Start program provides an important linkage between the family and a primary care provider, such as the pediatrician or general practitioner. The desirable outcome of such a linkage would see increased consultation and collaboration between home visitors and medical practitioners (Hawaii Department of Health, Maternal and Child Health Branch, 1994a).

The fourth component of the model is to identify family needs, facilitate referrals, and co-ordinate with other human service agencies for the family (Hawaii Department of Health, Maternal and Child Health Branch). According to Duggan, McFarlane et al., (1999) Hawaii Healthy Start programs continue "to improve parent and child outcomes in at-risk families by providing services directly and by promoting family use of preventive and early intervention services" (p. 67). Thus, Duggan, McFarlane et al. conclude the 
conceptual framework of Healthy Start remains faithful to the guiding principles of the original model.

The period 1981 to 1990 saw an increase in the number of child abuse and neglect cases reported for the State of Hawaii. Two probable explanations are offered by the Hawaii Department of Health, Maternal and Child Health Branch (1992):

There may be many reasons for the dramatic increase in the number of cases over the last decade. One of the reasons may be that in 1984 a new category "Imminent harm" was added which increased the total number of confirmed cases. This was also the time of increasing public awareness and action, with more cases of suspected abuse and neglect being reported for investigation. The fluctuations and leveling of the incidence figures between 1987 and 1990 may be an indication that these figures may not reflect the true incidence of child maltreatment. (p. 4)

Therefore, owing to significant perceived increases in abuse and neglect among children, a three-year pilot project was established in 1985. This demonstration project on the island of Oahu in the community of Ewa involved the provision of home visitations to high-risk families. During its period of operation, no cases of neglect or abuse were reported (Hawaii Department of Health, Maternal and Child Health Branch). This was out of a total enrolment of 234 parents, which represented $94 \%$ of the 248 families identified for services from the original screen of 1,693 families.

To augment these promising results a pretest-posttest design was developed for evaluation efforts. Program staff readministered the Family Stress Checklist developed by Kempe (1976) to parents and then compared the scores to results obtained when the child was born. The researchers ascribed a decline in test scores over time to the positive effects of the intervention. However, Duggan, McFarlane et al. (1999) contend that the original evaluation effort is flawed in at least two important ways. In readministering the Family Stress Checklist, program staff may have been biased due to their prior 
knowledge regarding the families' participation in the program. In addition, according to Duggan, McFarlane et al., because target families offered intervention services are identified with high scores on the Family Stress Checklist (Kempe), a relative decline in scores cannot be wholly attributed to intervention effectiveness due to statistical regression. Thus, "individuals defined by extreme scores on a measure at one time will show a shift in scores over time toward the average for all individuals even without the intervention" (Duggan, McFarlane et al., p. 69). Therefore, a decline in scores of the target families on the Family Stress Checklist is expected with or without an intervention.

Meanwhile, support for the program continued to grow because a reduction in child abuse and neglect had occurred. As legislators recognized the validity of the program model, budgetary allocations for program development increased annually from 1989 to 1995. Although a downturn in the state's economy led to a reduction in funding for Healthy Start programs, state support of the program continued. Due to the popularity of the original program, by 1991, Hawaii Healthy Start programs had expanded to over 13 sites statewide. As the program continued to report a reduction in child abuse and neglect, recommendations called for expanded screening to $90 \%$ of families with newborns, increased services up to five years of age, access to quality childcare, and access to preschool for all children three years of age (Hawaii Department of Health, Maternal and Child Health Branch, 1994a).

When a comprehensive evaluation of the scaled-up version of the program was completed involving randomized trials (National Center of Child Abuse Prevention Research, 1996), findings indicated: 
1. Healthy Start families provided a significantly more nurturing environment;

2. Mothers were significantly less punitive and restrictive of their children; and

3. Mothers had significantly reduced their risk for potential abusing, from moderate to low risk. (p. 25)

It was also concluded that reductions in the rates of child maltreatment occurred because of the paraprofessional home-visiting support program offered. Further, qualitative research yielded two other important findings. Parents who participated in the program obtained a better working knowledge of positive, nonphysical methods of child discipline, and parents received substantial benefits from their participation which mapped closely onto the goals specified by the program model (National Center on Child Abuse Prevention Research). Moreover, in conducting a thematic analysis of parent interview responses, three major themes emerged. The first theme found that parents believed they had benefited from the continued emotional and social support from the home visitor. The second theme indicated that parents had gained from the facilitation of access to additional services, such as medical or childcare. The third theme revealed consistent provision of information about child development and parenting skills (National Center on Child Abuse Prevention Research). As a result, findings of the study showed that Hawaii Healthy Start families provided a significantly more nurturing environment for their infants, mothers were demonstrably less punitive and restrictive of their children, and parents reduced their potential for abuse from moderate to low risk

Although both quantitative and qualitative research findings (National Center of Child Abuse Prevention Research, 1996) indicate positive results for the efficacy of Hawaii Healthy Start, it should be realized that home-visiting programs in themselves cannot address all the challenges that disadvantaged families face. Specifically, outcome measures as they relate to the cognitive development of the child and maternal social 
support remained unchanged after delivery of the Hawaii Healthy Start home-visiting program over the course of a year. This finding led researchers to conclude that homevisitation programs, integrated with service components directed towards developmental childcare, parent support groups, and personal skill building, might better serve the needs of disadvantaged families (National Center on Child Abuse Prevention Research).

However, Duggan, McFarlane et al. (1999) believe that previous research and evaluation efforts surrounding Hawaii Healthy Start are compromised. Specifically, Duggan, McFarlane et al. criticized the National Committee to Prevent Child Abuse study (NCPCA, 1993-1995) that reported results for a randomized trial as being limited methodologically, making the interpretations of the results and the reaching of conclusions difficult to achieve. Duggan, McFarlane et al. argue:

[The] execution of the NCPCA study proved challenging, with less-than-ideal followup combined with differential dropouts in the home-visited and control groups, an inability to blind interviewers to family group status, and reliance on program rather than evaluation staff to measure outcomes. (p. 70)

To rectify these concerns, a more rigorous evaluation of the Hawaii Health Start program was planned (Duggan, McFarlane et al.; Duggan, Windham et al., 2000). The study, conducted over a three year period from 1994 to 1999 , sought answers to the following research questions (Duggan, McFarlane et al.):

1. How well does actual program performance compare to the Healthy Start program model?

2. How successful is the program in achieving desired outcomes for parents and children?

3. How does fidelity of program implementation influence outcomes? How do benefits compare to direct and indirect program costs? (p. 71)

The authors contend that their study is one with a true experimental design, as there are three main study groups. The groups include the main control group, the Healthy Start 
program treatment group, and a testing control group. According to Duggan, McFarlane et al., the treatment and control groups are tested annually to establish outcome measures. To determine if the study's testing and data collection methods influenced any of the outcome measures, the control group for test effects was only assessed once at the end of three years (Duggan, McFarlane et al.).

The evaluation results reflected the delivery of the Healthy Start Program by three different agencies in six different sites. The agencies involved in the presentation of the program included: (a) a family social service organization, (b) Child and Family Services, (c) a health system agency dedicated to the prevention of child abuse and neglect, (d) the Hawaii Family Support Center, and (e) a grass roots family support organization called Parents and Children Together (Duggan, McFarlane et al., 1999; Duggan, Windham et al., 2000). What the results indicated is that program fidelity and service delivery had departed significantly from the original demonstration model that was tested over a decade ago (Duggan, Windham et al.). Differences were found to exist among the community agencies that implemented the model although they had similar contracts for service delivery from the state of Oahu. It is the belief of Healthy Start program network members that across-program variability in refusal rates and home-visitor frequency reflects differences in an agency's philosophy. According to Duggan, Windham et al.:

The agency with the highest number of visits but lowest percent of families active at 1 year views the entire family, more than the index child as its primary client. Thus, its home visitors are likely to concede to a family's change of heart about accepting home-visiting by closing cases when parents are uncertain they wish to continue in the program and focusing on families that are more receptive. The other two agencies expect that many at-risk families will be reluctant to engage in home-visiting but believe this underscores the need for continued outreach. They regard engagement of an isolated family as more important than complying with a family's inclination to be left alone. Thus, home visitors in these two agencies are encouraged to continue to try to engage reluctant families. (p. 257) 
Although the agencies have philosophical differences that can explain discrepancies in family engagement, there are also differences between agencies regarding home visitor ratings completed by the parent (Duggan, McFarlane et al., 1999). Nevertheless, all agencies have high ratings for home visitations, with Child and Family Services having the highest. According to Duggan, McFarlane et al., "agency differences in content of home visits, supervision, and the matching of home visitors and the family" (p. 76) account for the variability in satisfaction ratings. However, the researchers were unable to determine which one or combinations of these variables are attributable to interagency differences surrounding parent completed home-visitor ratings. Furthermore, Duggan, Windham et al. (2000) speculate that intersite differences in the frequency of home visits and the provision of core services are probably due to variation of organizational and program characteristics among the sites, the most notable being home-visitor knowledge and skill, staff turn over, and supervision (Duggan, Windham et al.).

Nonetheless, there are consistencies between agencies and among sites in the kinds of families that remain in the program and continue to receive services. It is found that not all difficult or high-risk families drop out, nor can it be concluded that only lower-risk families drop out. Rather, continuation in the program varied as a function of the risk factors associated with the parents. Duggan, McFarlane et al. (1999) report:

The results indicated that families were more likely to have had at least 12 visits if the father was violent, substance abusing, and at extremely high risk: if the mother had substance abuse problems; if the mother did not use violence unilaterally as a means of dealing with conflict with her partner; and if the mother was not at extremely high risk. (p. 76)

What is of interest is that there is no differentiation among agencies as to the types of families, as determined by risk factors, that remained in the program. However, mothers 
deemed to be at high risk or who were unilaterally violent towards their partner did not remain in the program. This is a concern because these mothers are at the greatest risk for child abuse (Duggan, Windham et al., 2000). These results indicate the value to program administrators in guiding their efforts if they better understand how program engagement varies as a function of parental risk factors (Duggan, Windham et al.). Furthermore, they also signal to program administrators and developers the necessity of determining overall program success in engaging and reaching subgroups that are at higher psychosocial risk (Duggan, Windham et al.).

The study then examines specific outcome measures for the program: (a) linking families to their communities, (b) improving child health and development, (c) affecting parenting behavior and attitudes, (d) bettering the home environment, and (e) reducing child maltreatment. To determine outcome measures, Duggan, McFarlane et al. (1999) incorporated standardized instruments that were valid and reliable in a-variety of populations. In examining interagency differences, it was found that "some Healthy Start program agencies succeeded in promoting certain aspects of child functioning and child development while others did not" (p. 84). Perhaps more revealing are differences among population subgroups. In examining both married and unmarried parents who were living together at baseline, the involvement and influence of fathers on their children in the Healthy Start program group show a trend towards greater accessibility than the control group but little difference in child rearing. However, if the subgroups are classified according to violent and nonviolent fathers, different program effects begin to emerge (Duggan, McFarlane et al.). Nonviolent fathers in the Healthy Start program group are more likely to see their children daily and have higher childcare scores. Although the 
previous analysis is only an illustration, Duggan, McFarlane et al. are committed to reporting through further analysis the implications of specific program impacts.

In determining how the present findings can influence future program development and evaluation, Duggan, McFarlane et al. (1999) identify eight different implications drawn of the study:

1. Even in a well established program, it is difficult to engage and retain families in home-visiting.

2. Traditional approaches to monitoring program output can give a false sense of security and mask deviations from the model.

3. Different agencies apply the same model differently, with varying success in family engagement.

4. Outcome assessment that focuses only on program participants is likely to be misleading: control groups are needed to estimate the value added.

5. Although home-visiting in Hawaii's scaled up program is less intense than that in the Healthy Start program model, there are positive effects for at-risk families.

6. Having a home-visiting program does not ensure effective linkage with community resources.

7. The evaluation of scaled-up programs should incorporate quality-of-care assessments.

8. The development of new evaluation strategies for countrywide intervention continues to require the accompaniment of strong scientific program evaluation. (pp. 85-88)

The findings presented by Duggan, McFarlane et al. have several important implications for paraprofessional home visitations. First, it is important to realize that evaluations of home visitations are often so focused on outcomes that the process of care is neglected. In a review of the literature conducted by Duggan, McFarlane et al., it was found that out of the 21 randomized trials of home visitations reviewed since the 1980 s, only 8 met the number of visits as advocated by the program protocol. Consequently, important process variables that need to be considered relate to the frequency of home visitations, the length of the home visits, attrition rates, and reasons for departure (Duggan, McFarlane et al.). Second, in conducting process evaluations of home visitations, it is important to consider 
not only the provider's perspective but also that of the family (Duggan, McFarlane et al.). Local values and needs must be considered in relation to how they effect implementation of the program model. Thus, it is ill-advised in Duggan, McFarlane et al.'s opinion, to adopt a home-visiting program simply because of its success in other settings without considering the context in which it is to be implemented.

Furthermore, because of advances in evaluation techniques, it is not appropriate to adopt methodologies that are circumspect and would not pass academic scrutiny. Although control groups are advocated for determining quantifiable outcome effects, Duggan, McFarlane et al. (1999) realize that not all home-visitation programs have access to resources required for large-scale randomized trials. Thus, Duggan, McFarlane et al. strongly recommend that program policy makers and planners should consider evaluation an integral component and make every effort to incorporate the strongest internal evaluation methodologies feasible. In this respect, although the effects of homevisiting programs might be promising in the first year, they need to be followed over time and to incorporate some form of cost-benefit analysis. As well, simply developing and implementing a home-visiting program does not necessarily ensure linkages with community resources. Rather, in Duggan, McFarlane et al.'s opinion, what is needed is service integration that "helps families negotiate services that are consistent and compatible in building on family strengths to achieve family-centered goals for healthy functioning and effective parenting" (p. 87). The final important implication for paraprofessional home visitations is an examination of the quality of service provided by the paraprofessional. 
To date many studies on Hawaii Healthy Start, including Duggan, McFarlane et al. (1999) and Duggan, Windham et al. (2000), tend to focus on program quality from a maternal measurement perspective. However, if the evaluation of service delivery is to be comprehensive and thorough, it needs to include a quality of care component. Future efforts to incorporate the development of new evaluation strategies need to maintain close links to scientific program evaluation in order to ascertain what does and does not work at the community level. In conclusion, Hawaii Healthy Start has grown significantly in the past 20 years. From pilot projects to scaled-up models, Hawaii has become a national leader in home-visitation programs for families at-risk. A continued commitment to improve evaluation efforts by studying one's experiences and sharing observations and lessons learned will lead to the sustained development of strong home-visiting interventions (Duggan, McFarlane et al.).

\section{Parenting Plus}

Parenting Plus was established as an early childhood development program of the Pipestone Health District in September 1999. Since then, it has continued to provide home-visitation services to families identified as overburdened. Because of health transition funding from the federal government and additional support to the program from the corporate sponsor SaskTel, Parenting Plus has been able to establish a core staff of three that includes a program co-ordinator and two family support workers (FSWs). The program co-ordinator, through a family assessment, identifies families that are at psychosocial risk, are overburdened, and would benefit from extra support. The FSWs then offer paraprofessional, strength-based home-visiting services to those identified 
families with newborns. Based out of the Grenfell Health Center, Parenting Plus serves the Pipestone Health District which includes the communities of Broadview, Fleming, Grenfel, Indian Head, Kendal, McLean, Montmartre, Moosomin, Qu'Appelle, Rocanville, Sintaluta, Wapella, Welwyn, Whitewood, and Wolseley. The area also includes the five First Nation communities of Carry the Kettle, Cowesses, Kahkewistahew, Ochapowace and Saskimay.

\section{Program Rationale}

In arguing for the provision of funding to establish Parenting Plus, a socioeconomic analysis of the indicators that affect the health and well-being of the parents of the Pipestone Health District was conducted (Pipestone Health District, 1998). Although scant data are available in relation to the national or provincial rates, the descriptive statistics are presented in Table 2.1. It is also reported that the Pipestone Health District has one-third fewer live births than the provincial average (Pipestone Health District). Given these indicators, it is argued that there are a number of residents who live in disadvantaged conditions. Consequently, if the needs of this special group go unnoticed, they will continue to experience inequities and are more likely to be less competent and confident parents. In turn, they may become nonproductive members of society "resulting in an increase in the use of health services, social services, education and justice services" (Pipestone Health District, p. 6). 


\section{Program Description}

Like Hawaii Healthy Start, the overriding goal of Parenting Plus is to assist communities and families in developing strategies for promoting optimal child development and improving family functioning. To attain this goal, Parenting Plus has been modeled after the Hawaii Healthy Start system of program delivery which is described within the context of four integrated components (Department of Health, Maternal and Child Health Branch, 1994a; 1994b). The first component relates to systematic screening and assessment of families through a two-stage screening process. The second element involves paraprofessional home visitations that use a strength-based approach to improve family functioning and reduce child abuse and neglect. This component includes parent skill building in the development of effective child-parent interactions, the modeling of coping skills, and the provision of emotional support to parents. The third program section relates to the establishment and maintenance of linkages between family, physician, public-health nurse, or community-health nurse. The fourth program constituent of the model seeks to maintain and establish referral to and co-ordination with community services for families. The third and fourth components of the model facilitate the delivery of integrated services to the family and augment the coordination of community-based human service programs to assist with the multifaceted needs of overburdened families for housing, respite care, day care, and financial crises, for example. 
Table 2.1.

Pipestone Health District Socioeconomic Indicators

\section{Living Arrangements/Families}

- Total number of census families in private households was 5,030.

- Total number of married and common law families, 4575 ( $90 \%$ of households).

- Total number of single parents, 455 ( $9 \%$ of all households).

- Total number of single parents not in labour force, 90 (19.8\% of single parents).

- Females 15 years and over with children under six years old, $490(57.6 \%$ employed; $44.9 \%$ unemployed).

- Population 15-24 years of age, 2,310 (57.6\% employed; $42.4 \%$ unemployed).

\section{Education}

- ages $15-24,2,295$ individuals with $43.6 \%$ of the population $(1,000)$ not attending school.

- Highest level of education ages 15 and over (<Grade 9, 19\%; Grade 9-12; 43.5\%; Grade 12 or greater $45 \%$ ).

Household Income

- Under $\$ 10,000,565$ (8.5\% of all households).

- $\$ 10,000-\$ 14,999,1100$ (15.8\% of all households).

- $\$ 15,000-\$ 19,999,830$ (11.8\% of all households).

Social Assistance

- 15 to 19 age group ( $4.2 \%$ of population).

- 20 to 29 age group ( $8.6 \%$ of population).

Source: Pipestone Health District, 1998.

\section{Program Screening}

Modeled after Hawaii Healthy Start, Parenting Plus utilizes a systematic two-stage screening and assessment process for early identification. The goal of the program is to provide universal screening of all newborns and to identify overburdened families residing in the Pipestone Health District. For Parenting Plus, this means the screening process includes the completion of a medical screen for physical and psychosocial risk status of the birth mother for all live births that occur for the district. As well, the 
Parenting Plus co-ordinator conducts a follow-up psychosocial assessment for those mothers receiving a positive screen (see Table 2.2).

By using a two-stage screening process, Parenting Plus is able to determine if the family is overburdened and eligible for home-visiting services. This is a unique aspect of the program where pro-active case findings with the Family Stress Checklist (Kempe, 1976) are used by Parenting Plus to identify those families in need of services before problems occur. Because of this, it is anticipated that the number of false positives and false negatives are reduced.

Table 2.2.

Parenting Plus Two-Stage Early Identification Screening

\section{Medical Record Screen}

1. Unmarried.

2. Partner unemployed.

3. Inadequate income.

4. Unstable housing.

5. No phone.

6. Education under 12 years.

7. Inadequate emergency contacts.

8. History of substance abuse.

9. Inadequate prenatal care.

10. Abortion unsuccessfully sought or attempted.

11. History of psychiatric care.

12. Relinquishment for adoption

13. Marital or family problems.

14. History of depression.

15 . History of abortions.

Medical Record Screen Scoring

Item scoring: True, false, unknown

Positive screen:

True score on either item number 1,9 , or 15 .

Two or more true scores

Seven or more unknowns
Family Stress Checklist Interview

1. Childhood history of being abused.

2. Substance abuse, mental illness or criminal history.

3. Previous or current child protection services involvement.

4. Low self-esteem, poor coping ability.

5. Multiple life stressors.

6. Potential for violent temper outbursts.

7. Unrealistic expectations for child's. development.

8. Harsh punishment of child.

9. Perceives child as being difficult or provocative.

10. Child unwanted or risk of poor bonding.

Family Stress Checklist Scoring

Item scoring for each parent: $0=$ No problem; $5=$ Mild; $10=$ Severe problem.

Positive assessment: A total score of 25 for either parent triggers referral to Parenting Plus.

Source: Pipestone Health District, 2001. 


\section{Program Delivery}

To help conceptualize program delivery and its resultant outcomes for Parenting Plus, a logic model was developed to illustrate the program's functioning. Briefly stated, logic models are increasingly being used in program evaluations and are thought to provide multiple benefits as they relate to a program's design and the conducting of ongoing and process evaluations. Essentially, a program's logic model links its essential components with its accomplishments thereby conveying the logical relationship between components and a program's expected outcomes (Conrad, Randolph, Kirby, \& Bebout, 1999; Julian, 1997; Julian, Jones, \& Deyo, 1995). Conrad et al. state that a logic model ought to encompass the program's context, its theory and the assumptions upon which it is predicated, the interventions being practiced, and the expected outcomes of the program.

In Conrad et al.'s (1999) discussion of logic models, context refers to the background conditions in which the program operates and which could have a moderating effect on the program's success. These include the geographic, economic, demographic, and political characteristics of the community where the program functions; regulations and policies that govern the program's operations; fiscal resources that finance the program; and community resources the program might access. Conrad et al. go on to state that the context may also include the target population who are served by the program. The theory or assumptions that underlie the program's intervention then refer to the theoretical construct that guides the design and the development of the intervention. The main activities are those components of the intervention that are assumed essential to achieve the intended outcomes. Finally, the outcomes are the effects of the intervention that are defined as short, intermediate, or long term. 


\section{Program logic model}

The program logic model for Parenting Plus details what activities are undertaken and what changes will result and for whom. Completing a program logic model for Parenting Plus helps to define the sequencing of actions and the prerequisites for change to occur. In turn, the logic model facilitates the ability to assess the likelihood of change occurring. The program logic model for Parenting Plus was derived through a review of the Healthy Start program design and consultation with the program staff of Parenting Plus on the prospects of change occurring (see Figure 2.5).

In the Parenting Plus logic model, as initially developed, medical record screens are completed while the mother is in the hospital with the newborn. If the medical record screen is positive, a family assessment with the Family Stress Checklist (Kempe, 1976) is conducted by the program co-ordinator. If either parent's total score on the Family Stress - Checklist is 25 or above, the family is offered home-visiting services. For those families who have not had a live birth but for whom a prenatal referral is made based on a positive medical record screen, an assessment with the Family Stress Checklist is also conducted. If a positive assessment results, the family is placed at Level $1 \mathrm{P}$, which designates prenatal service to the family. In providing prenatal care, the FSW and program coordinator determine the frequency and intensity of the services, with at least one home visit per month provided. Once the parent gives birth to the baby and is discharged from the hospital, the family is promoted to Level 1 .

Those families resistant to home visitations but who are still interested in participating in Parenting Plus and those families who have moved out of the area temporarily are placed on Level X. While on Level X, creative outreach is practiced for a period of two 
to three months unless the families become active participants. At the end of the two- to three-month period, a decision is made either to continue or to close the case depending upon the family's level of involvement.

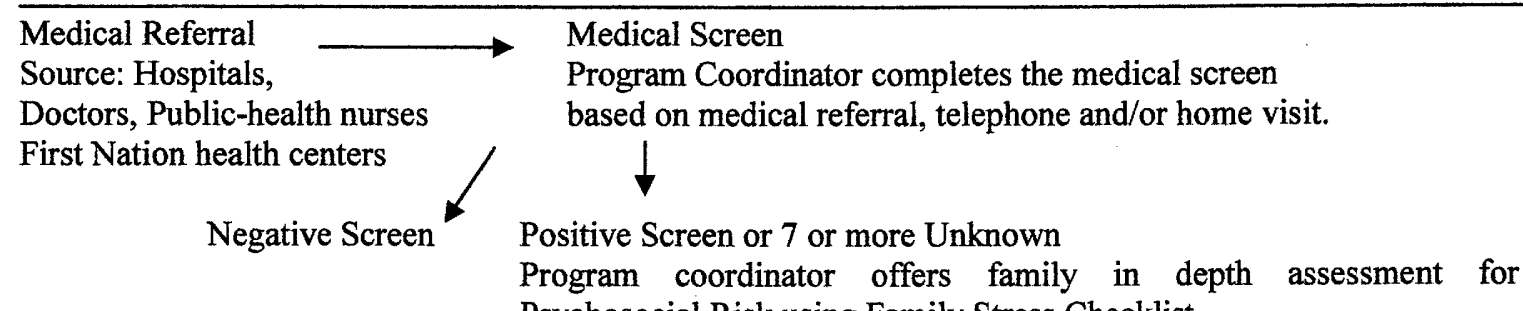
Psychosocial Risk using Family Stress Checklist

Negative Assessment Family not offered program

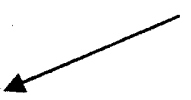

Positive Assessment Family refuses program Family cannot be contacted

If family interested but not committed To home-visiting family placed on Level X: Creative Outreach
Case conference to review home visitations

Document Family Changes in Home \& Telephone Visitation Record

Document level promotion

Implement Program Changes-Ongoing Document program changes in Family Support Plan<smiles>C[12CH3]</smiles>

Positive Assessment Family accepts program Family offered home-visiting<smiles>[Te]C1CC1</smiles>

If Prenatal family enters program On Level 1P: On birth \& care of child family placed at Level 1<smiles>CCC[Te]</smiles>

Family enters program at Level 1

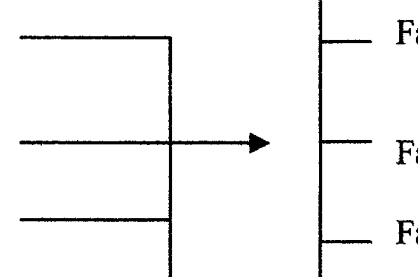

Family promoted to Level 2<smiles>C1CCCC1</smiles>

Family promoted to Level 3

Family promoted to Level 4

Family Discharged

In depth assessment for

Psychosocial Risk with

Family Stress Checklist
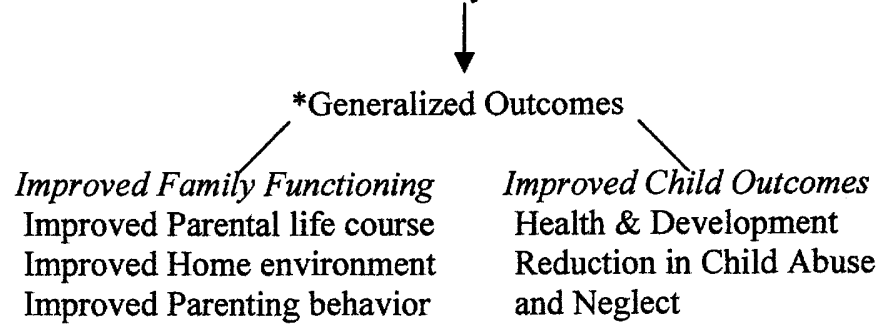

*Duggan, McFarlane et al., 1999. 
For those families accepting services and entering the program at Level 1, the first few months of home visitations are characterized by trust building and an attempt to engage family members. Once this has been established, the FSW completes an Individual Family Support Plan (IFSP) with the family and initiates the activities articulated in the plan. The FSW also observes parent-infant interaction, home safety, family dynamics, support systems, and coping skills and begins to teach and conduct parent-child interaction activities at this stage. If parents maintain stability in the home with no crises for a period of at least thirty days, and have kept over $75 \%$ of their appointments, are problem solving, expressing feelings and emotions appropriately, and are deemed to be responsive to parent child interventions, they are promoted to Level 2.

The middle phase of home visitations is characterized by Levels 2 and 3 and is the major intervention period, which can last up to 36 months. While on Level 2, the FSWs are required to make a home visit at least every two weeks. The FSW continues to observe and document parent child interactions, update the IFSP with the family, teach and conduct early childhood activities, and consult with the co-ordinator on a regular basis. Consequently, if the family has maintained stability in the home for 30 days with no crises; is using at least one positive support system; is demonstrating effective problem solving skills and child interaction skills; has demonstrated a reduction in one or more risk factors related to child abuse and neglect; and is scheduling well baby care visits and immunizations with their family doctor or the public-health nurse, it is promoted to Level 3.

Once a family is at Level 3, the FSW is to make at least one home visit per month to observe and document parent child interactions. At the same time, the FSW updates the 
family's IFSP and continues to teach and conduct parent child interaction activities. If a family has maintained stability in the home for thirty days with no crises; are using at least two positive support systems; are demonstrating effective problem solving skills and child interaction skills; has demonstrated a significant reduction in changeable risk factors on the Family Stress Checklist (Kempe, 1976); and is scheduling well baby care visits and immunizations with the family doctor or the public-health nurse, it is promoted to Level 4.

Once a family is at Level 4, the FSW is required to make a home visit every three months and record observations until the child enters kindergarten. The FSW continues to provide parent-child interaction activities according to program requirements and to monitor the child's welfare so as to make referrals and advocate for services needed to improve the child's health and development. If the family has maintained stability in the home for 30 days with no crises; is using at least two positive support systems; is demonstrating effective problem solving skills and child interaction skills; and is scheduling well-baby-care visits and immunizations with their family doctor or the public-health nurse, it remains on Level 4 until the target child is three years old or has entered kindergarten. Once the child is between three and five years of age and the family has met the previous criteria, they may be discharged from the program.

\section{Strength-based model}

The logic of the service delivery and the process by which participating parents progress is augmented by a strength-based model of care. Traditional models have come to be characterized by the professional or expert decision making process. It is argued 
that this type of approach is a deficit approach to a system of care because it is characterized by focusing on the situation and how to fix it. In contrast to the deficit model of service delivery is the strength-based approach in working with families. In describing the strength based philosophy and implications for program delivery Powell, Batsche, Ferro, Fox, and Dunlap (1997) state:

A strength-based philosophy is a critical belief, an all pervasive attitude, that informs all of the professional's interactions with families. It assumes that all families have strengths they can build on and use to meet their own needs, to accomplish their own goals, and to promote the well being of family members. The family-professional relationship starts not from an assessment of problems related to the child with a disability but from an attempt to fully understand the ways in which the family successfully accomplishes its goals and manages its problems. The professional can assist the family in using these strengths as building blocks and tools for enhancing not only the well being of the child with a disability but also the well being of all members. (p.4)

Consequently, within a strength-based model of service delivery like Parenting Plus, FSWs engage in establishing a partnership with the family that focuses on the parents' strengths and capacities and supports families in attaining their goals. Although Parenting Plus program protocol does call for assessment for psychosocial risk, it is clearly defined as a strength-based program.

\section{Program Resources}

Parenting Plus has developed program resources that include a memory book, cookbook, program brochure, and community resources pamphlet. The memory book is a type of baby book that is initially developed in conjunction with the family and FSW and then maintained by the family. Its purpose is to aid in establishing and building trust with families while providing a means for them to keep a record of their child's progress and explore their relationship with their child. A cookbook developed by Parenting Plus 
includes recipes and instructions on how to prepare baby food. As well, with support from the corporate sponsor SaskTel, Parenting Plus has produced a brochure to provide parents, the public, health care professionals, and early intervention stakeholders with information pertaining to the program and early childhood development.

\section{Expected Outcomes}

As a family-centered program, Parenting Plus intends to develop a broad based coalition of supports to promote the health and well-being of children and families they serve. Moreover, the intention is for families to increase their knowledge surrounding the healthy development of their children. It is hoped that the socio emotional, physical, psychological, and general health and resilience of the participating families and children will be improved (Pipestone Health District, 1998). By providing an integrated system of supports that are family-centered and strength-based; it is believed a model of service delivery for early intervention will be developed that is transferable to other sites in the province and country (Pipestone Health District).

\section{Summary}

Early intervention is a relatively new phenomenon. Prior to the $1960 \mathrm{~s}$, few intervention programs existed. The first early intervention programs targeted children with special needs and disabling conditions. These children were characterized as having intellectual or multiple disabilities and were usually labeled as being mentally retarded. The focus was on the amelioration of the disabling condition, following a medical or expert decision-making model, and the programs tended to be child-centered. 
However, Bronfenbrenner's (1979) work on the ecology of human development challenged this approach by advocating for interventions to be rooted in the ecology of the child. Bronfenbrenner believed that it is necessary to take a systems approach to the developing ecology that recognizes the multiple, interconnected systems that influence child development. Consequently, Bronfenbrenner's research established the theoretical underpinnings on which many of the different models for early intervention have been predicated. Because the scope of early intervention has been widened, a variety of services have come to be described as early intervention. Thus, not only are children with a special need or disabling condition considered as candidates for intervention but so are children and families at psychosocial risk. Moreover, services for children with special needs began to include the family in the services provided. More recently, early intervention efforts have adopted a strength-based approach to service delivery that is directed towards enabling families to more effectively utilize and access resources. The theoretical frameworks for early intervention that have developed in response to the demand for more comprehensive intervention services and which are based on Bronfenbrenner's theory of human ecology include: transactional regulation as developed by Sameroff $(1983,1993)$ and Sameroff and Fiese (2000a, 2000b); Guralnick's (1997, 1998) early development and risk-factors model; and Dunst and Trivette's (1997) resource-based approach to early intervention.

Briefly, transactional regulation theory tends to take more of a behaviorist approach to early intervention. The interventions tend to target children and families at psychosocial risk. According to Sameroff and Fiese (2000a, 2000b), the objectives of the interventions are to identify the strengths and weaknesses of parent-child transaction with 
the goal being to ameliorate dysfunctional regulatory systems. However, criticism of the model has emerged due to its behavioral focus, which is viewed as being too narrow in scope. Nevertheless, it should be remembered that transactional regulation as applied to early intervention is mainly directed towards children and families at psychosocial risk and that perhaps the characteristics of this special target population make interventions based on transactional regulation more amenable to treatment.

Responses to the inadequacies of intervention services for children with special needs or disabling conditions, while being influenced by Bronfenbrenner's theory of human ecology, also included Guralnick's $(1997,1998)$ model of early development and risk factors. Guralnick proposed that both risk and disability status affect the development of stressors that interfere with healthy patterns of family interaction and can result in negative child developmental outcomes. It is Guralnick's belief that early intervention efforts should target those stressors that affect healthy parent-child interactions. The program components that Guralnick identifies as being essential to the completion of this task include information and services, resource supports, and social supports. Of these three components, resource supports are viewed as most essential for families at psychosocial risk.

Partly in response to the perceived inadequacies of transactional regulation as too behaviorist in nature and due to the growing recognition that there are an increasing number of early intervention services for families who are at-risk, there has been the development of the resource-based approach to early intervention (Dunst \& Trivette, 1997). This approach to early intervention is characterized by shared decision making between client and service provider that concentrates on empowering of families by 
assisting them in recognizing their strengths. The resource-based model focuses on building partnerships with families through a strength-based approach. The components of the model through which early intervention services can be developed include sources of support, community resource mapping, and building community capacity. Although the resource-based model has existed for a number of years, it has not been adopted in its entirety by early intervention programs. Nevertheless, the strength of the model lies in its ability to aid families in recognizing and prioritizing their needs based on existing resources and sources of support available in the community.

Of the types of intervention services practiced, the most relevant to the present discussion are those that include a home-visiting component. In reviewing the type of services that have come to be described as home-visiting, one finds that not all programs are the same, and the factors that influence the type of service delivery developed include the anticipated and personal needs of the population, the professional expertise involved. in program design, resource availability, and program philosophical orientation (Ramey \& Ramey, 1993). The more generalized and comprehensive programs tend to be those that target low-income mothers and programs designed to prevent child abuse and neglect (Olds \& Kitzman, 1993). The reason why these programs are more all-encompassing is due to the complex and multifaceted demands that face this target group.

Special challenges that face home visitations, as revealed in the qualitative research literature, relate not only to the delivery of the program protocol, but also include maintaining access, facing environmental limitations, balancing professional and client responsibilities, matching maternal life course goals with child and family needs, and waiting for maternal readiness (Kitzman, Olds, et al., 1997). As well, it was found that 
process variables which can impact on the delivery of early intervention services include the integration of program theory with family characteristics and needs and how constrained resource allocation in the implementation phase of a program can change service delivery into a more reactive crisis driven mode.

Of the home-visiting programs that exist, the one that is most relevant to the present discussion is Hawaii Healthy Start, the model for Parenting Plus, the early childhood development program of the Pipestone Health District. Hawaii Healthy Start is an interesting program to examine not only because its main goal is to reduce child abuse and neglect but in the way that it approaches the problem. To affect positive parenting outcomes and the healthy development of newborns and children, Hawaii Healthy Start enhances parents' ability to utilize community-based resources. The present model of the program as implemented in the Pipestone Health District is viewed as being strengthbased. Although the original pilot program as based on Kempe's (1976) lay therapy was closely related to the theory of transactional regulation, recent implementations of the Hawaii Healthy Start model, including Parenting Plus, have seen the evolution of the program into a resource-based approach to early intervention.

Recent assessments of the program, (Duggan, McFarlane et al., 1999; Duggan, Windham et al., 2000) indicate that the focus of evaluation efforts needs to be directed towards implementation concerns. Evaluations should take into account the frequency of home visitations, the process of care from the client's as well as the service providers perspective, and the importance of local values and needs in relation to the program model implemented (Duggan, McFarlane et al.; Duggan, Windham et al.). It is incumbent on policy makers and program developers to include a comprehensive evaluation 
component and recognize it as integral to effective program implementation and development.

Thus, in acknowledgment of the disparities that exist in the Pipestone Health District as revealed by socioeconomic indicators and owing to the increased recognition of the benefits of early intervention in the research literature, a proposal for a paraprofessional home-visitation program modeled after Hawaii Healthy Start was drafted (Pipestone Health District, 1998). As a result of health transition funding, the establishment of Parenting Plus became a reality in September 1999. At that time, staff consisted of a program co-ordinator and two paraprofessional home visitors. As the program was being developed and implemented, concerns began to be expressed by stakeholders involved in early intervention in the health district regarding the program protocol and the model of service delivery being advocated. Moreover, difficulties in establishing partnerships and the signing of interagency agreements led to fewer parents being enrolled in the program. Lower than anticipated enrollment figures and the resignations of two key administrative positions involved in program development and implementation convinced stakeholders that an implementation evaluation of Parenting Plus was warranted. As a result, an implementation evaluation framework that is utilization-focused was adopted for the present study. 


\section{CHAPTER THREE}

\section{Methods and Procedures}

\section{Overview}

In assessing the evaluative needs for Parenting Plus, a series of stakeholder meetings that included the principal investigator were held over a 10 month period leading up to initial data collection. Each of the stakeholders involved in the meetings had an interest in the promotion of early childhood intervention in the province and represented various sectors, including non-profit, corporate, educational, and governmental organizations. Stakeholders participating in the development of the evaluation framework for Parenting Plus included: Saskatchewan Health, community care consultant; the director of Population Health, Pipestone Health District; program co-ordinator and the family support workers (FSWs) for Parenting Plus; Department of Educational Psychology and Special Education faculty members; the Saskatchewan Institute on the Prevention of Handicaps (SIPH) director and research officer; marketing co-ordinator for the corporate sponsor SaskTel; and the principal investigator.

Initial discussions surrounding the evaluative needs of Parenting Plus began on October 15, 1999, when Saskatchewan Health sponsored a conference call with evaluation stakeholders. Parenting Plus, the home-visitation program of the Pipestone Health District, had started up in September 1999 with the hiring of program staff. Because of the conference call, stakeholders in the evaluation, 
decided to reconvene at a round table meeting on December 1, 1999, hosted by the SIPH in Saskatoon. Stakeholders in attendance at this round table meeting, at the Kinsmen Children's Centre, included: the director of marketing for Sask Tel; the community care consultant for Saskatchewan Health; the director and research Officer for SIPH, the Director of Population Health for the Pipestone Health District; the Parenting Plus program co-ordinator and FSWs; faculty members from the Department of Educational Psychology and Special Education, University of Saskatchewan; and the principal investigator.

At this meeting stakeholders discussed the model of program delivery, the status of the program as implemented and the expected outcomes. Based on this dialogue, an evaluation framework was drafted by the principal investigator and presented to stakeholders at the next meeting on February 15, 2000 and hosted by the SIPH. In attendance at this meeting was the director of population health for the Pipestone Health District; the executive director and the research officer for SIPH; the community care consultant for Saskatchewan Health; faculty from the Department of Educational Psychology and Special Education at the University of Saskatchewan; and the principal investigator. The evaluative framework that evolved from the December meeting was a quasi-experimental time series study that included measures pertaining to family functioning, parenting styles, and child development. However, at the February meeting, the director of population health for the Pipestone Health District disclosed that difficulties pertaining to the implementation of the program were being encountered, and the number of participating families was lower than anticipated. Stakeholders in the evaluation 
began to question the efficacy of conducting a summative evaluation that focused on outcome measures. In part, discussion centered on how evaluation efforts should be redirected towards the formative aspects of the program and more specifically the implementation process. Stakeholders decided that the next meeting, scheduled for March 16, 2000, would result in an implementation evaluative framework.

In attendance at the March 16 meeting, hosted by the Department of Educational Psychology and Special Education at the University of Saskatchewan, were faculty members from the department; the community care consultant for Saskatchewan Health; the director of population health for the Pipestone Health District; and the principal investigator. At this meeting, the director of population health for the Pipestone Health District revealed that the Parenting Plus program co-ordinator had resigned. This confirmed the need for an implementation evaluation of the program. However, shortly thereafter the director of population health for the Pipestone Health District, who was the principal stakeholder for the evaluative needs of Parenting Plus, left the district to assume a position as a CEO for another health district in the province. With the departure of two key administrative positions for Parenting Plus, stakeholders decided to postpone further discussions surrounding the evaluative needs of the program until the new staff had an opportunity to acquaint themselves with the Pipestone Health District. Shortly thereafter, on August 24, 2000, a stakeholder meeting was held, hosted by Saskatchewan Health in Regina, and involving the community care consultant for Saskatchewan Health; the new program co-ordinator and director of population health for the Pipestone District; Parenting Plus FSWs; and the principal investigator. At this time, details pertaining 
to the implementation evaluation framework were reviewed. Data collection would occur over a four month period beginning in September 2000 and ending in December 2000. Feedback would be solicited informally by the principal investigator to ensure trustworthiness of the data and, formally, by providing to Parenting Plus for review a preliminary report of the evolving themes and descriptive statistics by October 31,2000 , followed by a draft copy of the summary report by January 2001 .

The purpose of the evaluation framework meetings was to define and clarify the evaluation from the perspective of intended users and stakeholders. Furthermore, as Parenting Plus is a pilot program, stakeholders decided that evaluation efforts be directed towards process as opposed to outcome variables. Stakeholders indicated that information pertaining to the implementation process for Parenting Plus to inform subsequent and present efforts was needed. In the operationalization of an implementation evaluation, Patton (1990) provided the stakeholders with one of the more salient descriptions:

One important way of studying program implementation is to gather detailed, descriptive information about what the program is doing. Implementation evaluations answer the following kinds of questions: What do clients in the program experience? What services are provided to clients? What does staff do? What is it to be like in the program? How is the program organized? As these questions indicate, implementation evaluation includes attention to inputs, activities, processes, and structures. (p. 105)

This type of approach towards evaluation is described as utilization-focused (Patton, 1986, 1997a). This is because the purpose of the stakeholder evaluation meetings for Parenting Plus was to define and clarify the evaluation from the perspective of intended users. 
Thus, during the meetings in March and August of 2000, stakeholders decided that in conducting an implementation evaluation three main areas of investigation needed to be undertaken. The first goal is to understand the model of service delivery that has emerged and been operationalized for Parenting Plus. Second, the evaluation ought to determine the characteristics of the implementation process that either facilitated or hindered the development of Parenting Plus. Finally, the third objective is to gain insight into the parents' experience and perspective of Parenting Plus.

The implementation evaluation for Parenting Plus was undertaken within the guiding utilization-focused framework. The information derived informed present and subsequent efforts by reporting on the fidelity of program implementation and the model of program delivery operationalized, while at the same time integrating the experiences of participants and stakeholders. The summary findings would then be integrated into a conceptual model depicting the operationalization of the program.

\section{Evaluation Framework Rationale}

Due to the general failure of experimental or quasi-experimental methodologies to provide information that is both timely and useful, program evaluation has seen the development of a genre of evaluation methodologies labeled "pragmatism" (Greene, 1994). Perhaps one of the best-known proponents of this type of evaluation is Patton's $(1986,1997$ a) utilization-focused evaluation, which creates a match between the intended uses of the evaluation and the evaluation process. Fishman 
(1997), in his critical review of Greene's (1994) chapter on program evaluation and her representation of the four general social science paradigms applied to program evaluation, contends that the pragmatism paradigm, as formulated by Greene, contains elements of post positivism, constructivism, and critical theory. Given that pragmatism encompasses such diverse ontologies within one epistemology, it would seem, by adopting such an approach, that contradiction and tension would be inherent. However, program evaluation is an applied study and does not set out to prove or disprove theory. As such, the aim of pragmatism in program evaluation is to satisfy the objectives and needs of the intended users by providing timely and useful information, instead of trying to find justification in a philosophical paradigm necessary for theory building.

Characteristic of pragmatism, as represented in program evaluation, is the orientation of evaluative methodologies that reflect the needs and questions of management and the various stakeholders (Patton, 1986, 1997a). Eclectic methodologies are employed and the evaluator's focus is on effective program management. According to Patton (1997a), the defining question or approach to utilization-focused evaluation asks, "What information is needed and wanted by primary intended users that will be used for program improvement and decision making" (p. 89)? Patton advances the argument that because utilization-focused evaluation attempts to match the uses of the evaluation with its users, it is a menusoriented approach.

In a menus-oriented approach, the methodologies employed to best answer the research question posited can often be diverse in nature and encompass both 
quantitative-experimental methods and qualitative-naturalistic approaches (Greene, 1994; Fishman, 1997; Patton, 1997a). The menus offered by Patton for focusing an evaluation list various options matched with the major questions or approaches. $\mathrm{He}$ cautions that his list is not exhaustive and that the various options offered for focusing the evaluation be used together or sequentially in the same evaluation. According to Patton (1997a):

Various options can be and often are used together within the same evaluation, or options can be implemented in sequence over a period of time, for example, doing implementation evaluation before doing outcome evaluation, or formative evaluation before summative evaluation. (p. 192)

Utilization-focused evaluation then may include any one of the 58 different types or focuses listed in Patton's menu. Perhaps this is why the methodologies he employs and advocates are described as eclectic in nature. Greene (1994), in her discussion of program evaluation and pragmatism, comments on Patton's work. In discussing the rise of pragmatism, as typified by utilization-focused methods, Greene (1994) states:

The second genre of evaluation methodologies [pragmatism] grew in response to the failure of experimental science to provide timely and useful information for program decision making. Characteristic of these methodologies are their orientation to decision making and hence to management, their primary emphasis on producing useful information, their practical and pragmatic value base, and their eclectic methodological stance. (p. 532)

Naturally, Patton (1997a) does not reject combining quantitative and qualitative methodologies, as he views the incorporation of both methods in an evaluation as an enhancement to the knowledge base. Moreover, in attempting to delineate a paradigm that would provide the most appropriate methodology or mix of methodologies in qualitative and quantitative studies, Tashakkori and Teddlie (1998) believe that pragmatism is probably the best approach. They argue for its use 
in mixed methods designs:

The pragmatism point of view is illustrated as rejecting the forced choice between positivism (including post positivism) and constructivism with regards to methods, logic, and epistemology. In each case, pragmatism rejects the eitheror of the incompatibility thesis and embraces both points of view. (p. 23)

Tashakkori and Teddlie remind the researcher that the research question asked and how best to answer it are the guiding criteria in deciding what epistemology and ensuing methodology to incorporate in a given study.

\section{Implementation Evaluation}

Based on the evaluative needs of Parenting Plus stakeholders, a utilizationfocused implementation evaluation was undertaken. In characterizing the evaluation framework for an implementation evaluation and the methods employed, Patton (1990) states:

If program implementation is characterized by a process of adaptation to local conditions, needs, and interests, then the methods used to study the implementation must be open ended, discovery oriented, and capable of describing developmental process and program changes. (p. 106)

Patton (1997a), in discussing the options and variations available for an implementation evaluation, contends that there are five main types. He argues that they be used in combination or alone, depending on the intended uses of the evaluation.

According to Patton (1997a), the five types of implementation evaluation include: effort, monitoring, process, component, and treatment specification. First, effort evaluation refers to the creation of an inventory that documents the quality and quantity of implementation efforts. Monitoring evaluation from an 
implementation perspective refers to the establishment of a management information system that documents and "provides routine data on client intake, participation levels, program completion rates, caseloads, client characteristics, and program costs" (Patton, 1997a, p. 205). Component evaluation refers to a formal assessment of the various components of a program in order to facilitate greater cross-program comparability and generalizability. Treatment specification evaluation, within an implementation context, refers to the identification from an experimental perspective of the causal assumptions that undergrid the program and its resultant effects. Treatment specification attempts to delineate the independent and dependent variables and how they influence outcomes. Finally, and most relevant for Parenting Plus, are process evaluations. According to Patton (1997a): "Process evaluation focuses on the internal dynamics and actual operations of a program in an attempt to understand its strengths and weaknesses" (p. 206).

Not new to program evaluation, process evaluations were first developed in the CIPP model for educational accountability by Stufflebeam $(1971,1972)$. The CIPP model contains four integral components that include context, input, process, and product, and attempts to match these components with decision-making. Stufflebeam developed the CIPP model to aid in proactive decision making in educational program evaluation. He contends that the type of decisions specific to the model include planning decisions for the obtainment of project objectives, structuring decisions that relate to a program's design, implementation decisions that specify how a program design is to be operationalized, and recycling decisions that refer to the modification or continuing of a program. What is unique about the 
CIPP model is that it links the four types of evaluation to the type of decision each serves (see Figure 3.1).

\begin{tabular}{|c|c|c|c|}
\hline \multicolumn{2}{c}{$\begin{array}{c}\text { Evaluation Types } \\
\text { Input }\end{array}$} & Process & Product \\
\hline Objectives & $\begin{array}{c}\text { Solution } \\
\text { Strategy } \\
\text { Procedural } \\
\text { Design }\end{array}$ & Implementation & $\begin{array}{c}\text { Termination, } \\
\text { continuation, } \\
\text { modification, } \\
\text { or installation. }\end{array}$ \\
\hline $\begin{array}{c}\text { Record of } \\
\text { objectives and } \\
\text { bases for their } \\
\text { choice. }\end{array}$ & $\begin{array}{c}\text { Record of } \\
\text { chosen strategy } \\
\text { and design and } \\
\text { reasons for their } \\
\text { choice. }\end{array}$ & $\begin{array}{c}\text { Record of the } \\
\text { actual process. }\end{array}$ & $\begin{array}{c}\text { Record of } \\
\text { attainments } \\
\text { and recycling } \\
\text { decisions. }\end{array}$ \\
\hline
\end{tabular}

Figure 3.1 Relevance of the CIPP Model to Decision Making and Accountability Source: Stufflebeam, 1972, p. 5.

According to Stufflebeam (1972): -

Context evaluation provides information about needs, problems, and opportunities in order to identify objectives. Input evaluation provides information about the strengths and weaknesses of alternate strategies for achieving given objectives. Process evaluation provides information about the strengths and weaknesses of a strategy during implementation so that either the strategy or its implementation might be strengthened. Product evaluation provides information for determining whether objectives are being achieved and whether the procedure employed to achieve them should be continued, modified or terminated. (p. 3)

The relevance of a process evaluation from an implementation perspective is that it allows for an examination of the implementation of the project by documenting the process and determining if the program implemented and operationalized was the one originally designed. Furthermore, if the program is not similar to the one intended, a process evaluation aids in determining if this is due to an inadequacy in 
the design or to other reasons that affected the implementation process. From a utilization-focused perspective, this type of information is relevant to the evaluation of Parenting Plus because it seeks to find answers related to stakeholders needs.

In understanding how the evaluation framework relates to the objectives of the study, it is important to realize that heavy emphasis is placed on process evaluation. However, the evaluation also includes monitoring and component evaluation within an implementation framework. The monitoring aspect of the study relates to the characteristics of service delivery and the establishment of base rates for the program. Furthermore, as Parenting Plus is modeled after Hawaii Healthy Start, the objectives of the study also seek to understand how the program components, as they pertain to a) screening and assessment, b) paraprofessional home visitations, c) informal community support, and d) mechanisms for the co-ordination of services and supports, are implemented and operationalized. Finally, the study, seeks to determine what participants' perceptions of the program are and what they actually experienced in the program. From an implementation perspective and as one of the four key components of the program, it is important to gain an understanding of the home visitations from the participants' perspective. In turn, characteristics of the implementation and operationalization of Parenting Plus are obtained. Within this context a utilization-focused evaluation framework that uses a mixed-methods approach, rooted in the epistemology of pragmatism and ontology of constructivism, was designed. 


\section{Methodological Rationale}

As a utilization-focused evaluation, both quantitative and qualitative methods are used to collect data. The objective of the evaluation is to develop an understanding of the model of service delivery that has evolved, the characteristics of the implementation process, and how participants experienced Parenting Plus. As an applied research study, there is no attempt to generate or create theory, as would be the case if the methodology employed were grounded theory. Nevertheless, there are a number of similarities between the present study and grounded theory. Although there is no attempt at theory generation and its specific components (a central phenomenon, causal conditions, strategies, conditions, and context) as there would be in a grounded theory study, the methods used are best viewed within this genre. This is because the evaluation of Parenting Plus is an attempt at creating an understanding of the implementation and operationalization of Parenting Plus. Although there is no attempt at theory generation, this type of approach is inductive in nature.

One of the earliest proponents of the inductive approach was Patton (1990) who described it within the context of data analysis for program evaluations. Since then, inductive analysis has been reported widely within the research literature. Research with the inductive approach has been found in the areas of disability (Campbell \& Jones, 2002; Guteng \& Chappell, 2000), education (Dyson \& O'Sullivan, 1998; LeMare \& Sohbat, 2002; Shepardson, 1996), health care (Backett \& Davison, 1995; Pope, Ziebland \& Mays, 2000), and child abuse programs (Socolar, Fredrickson, Block, Moore, Tropez-Sims, \& Whitworth, 2001). Recently, Thomas has applied 
the inductive approach to health and community care program evaluations that include a mixed-method outcome evaluation for a paraprofessional home-visitation program currently offered in New Zealand and modeled after Hawaii Healthy Start (D. R. Thomas, personal communication, April 23, 2002).

The general inductive approach has evolved because of the need by researchers to use a specific set of principles in their data analysis, regardless of their epistemological assumptions Thomas (2000). There are many similarities between grounded theory and the general inductive approach. The intent and purpose of the general inductive approach is to allow the emergence of themes and categories from the raw data and, in this sense, it is similar to grounded theory. However, the general inductive approach differs from grounded theory in that it does not prescribe the use of the constant comparative method of data analysis, which is a systematic process characterized by theoretical sampling, open coding, and axial-coding, in an attempt to generate or discover a theory about the phenomena under investigation.

Through the summary of a number of qualitative reports that have not labeled what they have done within one of the identifiable traditions of qualitative research, a procedure for conducting qualitative analysis based on the objectives of the study has been developed (Thomas, 2000). These are the key features or objectives of the general inductive approach according to Thomas:

1. To condense extensive and varied raw text into a brief, summary format.

2. To establish a clear link between the research objectives and the summary findings which are to be derived from the raw data and to ensure these links are both transparent (able to be demonstrated to others) and defensible (justifiable given the objectives of the research).

3. To develop a model or theory about the underlying structure of phenomena or processes which are evident in the text (raw data). (p.3) 
Consequently, Thomas has developed a set of underlying assumptions upon which the general inductive approach is predicated (see Table 3.1).

Table 3.1

Underlying Assumptions of the General Inductive Approach

1. Data analysis is determined by both the research objectives (deductive) and multiple readings and interpretations of the raw data (inductive). Thus the findings are derived from both the research objectives outlined by the reseracher(s) and findings arising directly from the analysis of the raw data.

2. The primary mode of analysis is the development of categories into a model or framework that summarizes the raw data and conveys key themes and processes.

3. The research findings result from multiple interpretations made from the raw data by the researcher who codes the data. The findings are inevitably influenced by the perspectives, assumptions and experiences of the researchers who conduct the research and who carry out the data analysis. In order for the findings to be usable, the researcher (data analyst) must make decisions about what is more important and less important in the data.

4. That different researchers are likely to produce findings which are not identical and which have non-overlaopping components.

5. That the trustworthiness of findings can be assessed by the extent of consistency when compared with related findings. Related findings might be derived from (a) independent replication of the research, (b) comparison with findings from previous research, (c) triangulation within a project, (d) feedback from participants in the research, and (e) feedback from users of the research findings.

Source: Thomas, 2000, p. 4.

Unlike grounded theory that would impose restraints and create the need for justifications that might be untenable, the general inductive approach is most appropriate for program evaluations. This is because program evaluations like 
Parenting Plus are applied studies that do not concern themselves with theory generation. As the evaluation framework for Parenting Plus is an applied study of the implementation process, the general inductive approach to qualitative data analysis is used for the qualitative data collected for its implementation evaluation.

In undertaking an inductive analysis, the raw-data are summarized into categories from which themes and processes are developed (Thomas, 2000). In describing the key features of the coding categories, Thomas explains that a particular study can potentially have five features: label for a category, description of a category, text or data associated with the category, links, and the type of model in which the category is embedded (see Table 3.2). In the reporting of findings, the summary or top-level category labels are main headings illustrated with quotations of raw-data (Thomas). The establishment of the linkages between the various superordinate, subordinate, and parallel categories develops the framework or model. The connections between the coding categories are based on commonalities between the classifications. Once the similarities and dissimilarities of the various categories are established, a hierarchal or tree model, for example, can be created (Thomas). The final key feature of the coding categories relates to the identification of where the model, framework, or theory is situated. To illustrate how results were derived using the general inductive approach for the semi-structured depth interviews, the first-order sub category, Lack of Feedback for the major dimension, Partnership Building and Communication, is presented (see Table 3.3). 
Table 3.2

Five Key Features of Coding Categories

\begin{tabular}{|l|l|}
\hline \multicolumn{1}{|c|}{ Component } & \multicolumn{1}{|c|}{ Description } \\
\hline Label for category & $\begin{array}{l}\text { Word or short phrase used to refer to category. } \\
\text { The label often carries inherent meanings which } \\
\text { may not reflect the specific features of the } \\
\text { category. }\end{array}$ \\
\hline Description of category & $\begin{array}{l}\text { Description of the meaning of category including } \\
\text { key characteristics, scope and limitations. }\end{array}$ \\
\hline Text or data associated with category & $\begin{array}{l}\text { Examples of text coded into category which } \\
\text { illustrate meanings, associations and perspectives } \\
\text { associated with the category. }\end{array}$ \\
\hline Links & $\begin{array}{l}\text { Each category may have links or relationships } \\
\text { with other categories. In a hierarchical category } \\
\text { systems (e.g. tree diagram) these links may } \\
\text { indicate super ordinate, parallel and subordinate } \\
\text { categories (e.g. parent sibling, child). Links are } \\
\text { likely to be based on commonalities in meanings } \\
\text { between categories. }\end{array}$ \\
\hline Type of model in which category is embedded & $\begin{array}{l}\text { The category system may be seen as one of } \\
\text { several different types of models, theories or } \\
\text { frameworks. These include: an open network (no } \\
\text { hierarchy or sequence), a temporal sequence (e.g. } \\
\text { movement or time), or a casual network (one } \\
\text { category causes changes in another). It is also } \\
\text { possible that a category may not be embedded in } \\
\text { any model or framework. }\end{array}$ \\
\hline
\end{tabular}

Source: Qualitative data analysis: Using a general inductive approach (Thomas, 2000).

The results obtained through the inductive approach to data analysis, represent the emergence of the major dimensions that have evolved out of the semi-structured depth interviews. Although multiple readings of the text allow for the emergence of units of meaning and eventually categories, the approach is also deductive in nature in that the research objectives of the evaluation influence the parameters by which data analysis is undertaken. 
Table 3.3

Example of Coding Category

\begin{tabular}{|c|c|}
\hline Component & Description \\
\hline Label for Category & Lack of Feedback \\
\hline Description of Category & $\begin{array}{l}\text { Category describes ineffective communicati } \\
\text { practices evidenced in providing feedback } \\
\text { stakeholders surrounding the program. }\end{array}$ \\
\hline Text or data associated with category & $\begin{array}{l}\text { I would like to hear something, that's why I } \\
\text { saying if there was something wrong I'm sure s } \\
\text { would let me know so I'm just assuming things ' } \\
\text { okay, if she doesn't communicate with me. Feedbc } \\
\text { on the positive side of it that is the only thing tha } \\
\text { think is lacking. We are communicating now, it's } j \\
\text { a little better communication and explaining } \\
\text { positive side of the program. And having a little bit } \\
\text { feedback in how they are doing with the families. } \\
\text { knew some statistics or knew anything, like even } j \\
\text { for the workers to pop in and tell me how it's gol } \\
\text { with the clients they're working with in } \\
\text { community or any kind of feedback or numbers wor } \\
\text { be good. I think the bottom line is communication a } \\
\text { just reinforcement and sending out positive messas } \\
\text { you know, the program is up and running, this is h } \\
\text { many clients we've got, we feel it is being successy } \\
\text { appreciate your help or whatever. I think it is } j \\
\text { relationship management. You know it is picking } \\
\text { the phone once a month and saying-oh hi I am } j \\
\text { calling to report on whatever, and firing off a qu } \\
\text { fax or email of the latest, updated stats. }\end{array}$ \\
\hline Links & $\begin{array}{l}\text { Lack of Feedback is one of three first-or } \\
\text { subcategories that comprise the second-order s } \\
\text { category, Ineffective Communication, represent } \\
\text { under the major theme, Partnership Building a } \\
\text { Communication. The other two first-order categor } \\
\text { subsumed under Ineffective Communication inclu } \\
\text { Poor Role Clarity and Poor Pre Implementat } \\
\text { Communication, with Ineffective Partnersl } \\
\text { Building, the other second-order category represent } \\
\text { under the major theme, Partnership Building a } \\
\text { Communication. }\end{array}$ \\
\hline Type of model in which category is embedded & $\begin{array}{l}\text { Semi-structured depth interviews within an impli } \\
\text { implementation casual framework. }\end{array}$ \\
\hline
\end{tabular}

Data were collected over a four month period commencing in September 2000 and concluding in December 2000. The qualitative methods used in the evaluation included semi-structured depth interviews and focus groups modeled after 
Fetterman's $(1996,1997,1999)$ empowerment evaluation (EE). The quantitative methods used in the evaluation included analysis of data gleaned from the Parent's scale of the Family-Centered Program Rating Scale (FamPRS) (Murphy, Lee, Turbiville, Turnbull, \& Summers, 1991) and a document review that abstracted rates of service delivery. Quantitatively, descriptive statistics were generated from the document review that included frequency distributions and percentages in an effort to establish base rates for the program. The FamPRS data resulted in the generation of descriptive statistics. The benefit of using the FamPRS was that it provided a quantitative measure of the parent's satisfaction with Parenting Plus and aids in data triangulation by source. Thus, based on a methodological rationale that used the general inductive approach to qualitative data analysis, a mixed methods study cast within the parameters of a utilization-focused implementation framework was undertaken.

\section{Interviews}

Semi-structured depth interviews were conducted in person and on the telephone with stakeholders. This approach allowed for the exploration of themes relevant to program implementation and the stakeholder's evaluative needs. Wengraf (2001) argues that the definition of "depth" is comprised of two interrelated components:

1. To go into something "in depth" is to get more detailed knowledge about it.

2. To go into something in depth is to get a sense of how the apparently straightforward is actually more complicated, of how the "surface appearances" may be quite misleading about "depth realities". (p. 6) 
Furthermore, in describing semi-structured interviews as being depth interviews, Wengraf explains that the concept of depth can also be recast as one of width. Thus, in conceptualizing the operationalization of depth, it is clear that its salient features encompass a breadth of understanding that goes beyond surface meaning to discover knowledge-based findings about reality.

In the design of semi-structured interviews to achieve depth and elicit meaning, it is important to have prepared questions relevant to themes that the researcher wants to explore (Kvale, 1996; Wengraf, 2001). A central purpose drives the development of research questions that in turn guides the development of theory building or theory testing questions. However, the language used in interviews is not the same as the language used in defining a study's research purpose and research questions (Kvale; Wengraf). According to Wengraf (2001):

The theory-questions "govern" the production of the interviewer-questions, but the theory-questions are formulated in the theory-language of the research community, and the interview-questions are formulated in the language of the interviewee. (p. 62)

Moreover, Kvale describes the relationship between the research question and interview question along two interrelated dimensions that are dynamically and thematically defined. Like Wengraf, he argues that there needs to be a thematic relationship between the research and interview question but that this relationship should be a dynamic one in order for there to be a positive interaction and flow to the interview. What is of interest in Kvale's discussion is the recognition that the type of interview questions developed for the study can affect the interaction between the interviewer and interviewee. Although Kvale (1996) notes, "A good thematically based research question need not be a good dynamic interview 
question" (p. 130), it is important that the questions for the interview reflect the ability to derive thematic knowledge while at the same time engendering a naturalistic, dynamic flow to the conversation. Moreover, the interview questions are not mutually exclusive to a particular research question, as meaning derived from the interview is multilayered and does not reside within a categorical description. For the implementation evaluation of Parenting Plus, the research questions surrounding the evaluation are derived from the research literature on implementation evaluations and stakeholders' information needs. Samplings of the parallel construction between research and interview questions for the implementation evaluation of Parenting Plus are outlined in Table 3.4.

As well, the questions in semi-structured interviews must be sufficiently open ended to elicit an informant's response. In turn, the interviewer cannot predict what the responses will be and, therefore, some of the interviewer's subsequent rejoinders are extemporized. Wengraf (2001) states:

Semi-structured interviews are designed to have a number of interviewer questions prepared in advance but such prepared questions are designed to be sufficiently open that the subsequent questions of the interviewer cannot be planned in advance but must be improvised in a careful and theorized way. (p. 5)

In this sense, the role of the interviewer is to allow for the exploration of themes by the interviewees but not to influence opinion on such themes (Kvale, 1996). Kvale explains that characteristic of the semi-structured interviewing process are specific types of questions asked by the interviewer for clarification. These include introduction and follow-up questions, specifying questions, direct questions and indirect questions, structuring questions and interpreting questions, and silence. 


As Parenting Plus has been implemented, what is the
model of service delivery that has emerged and been
operationalized?

What characteristics of the implementation process for Parenting Plus have facilitated or hindered the systematic screening of high-risk families of newborns, the provision of paraprofessional home visitations for those families, the development of informal community supports for families, and the mechanisms for the co-ordination of supports and services?
What do the participants' experience and what do they understand about the paraprofessional home visitations they receive from Parenting Plus?
What is your understanding of how Parenting Plus operates in the Pipestone health district?

What do you see as your primary responsibilities and have these responsibilities changed?

How do you believe this program has an effect on services that are being delivered to the target group (changed, enhanced, or new services being developed)?

What are the procedures identified and implemented so that members of the designated population are given the opportunity to access the program?

What resources were anticipated for full implementation?

Is the governance structure in place and did it provide the support and linkages required?

What has been learned about implementation of this program that might inform similar efforts elsewhere?

Describe your working relationship with the Pipestone Health District and the Parenting Plus program staff and what factors, positive or negative have contributed to your ability to work together?

What do participants actually do in the program?

What do participants like or dislike?

What are the specific strategies used to work with, interact with, or involve participants?

What are the program's key characteristics as perceived by you?

What are the short-term effects of Parenting Plus? 
To illustrate the mode of questioning that occurred for the semi-structured interviews of the study, a verbatim transcript of the various questioning styles is cited in the excerpts from the following interview. The interview is of a health care professional who has been involved with Parenting Plus because of her role with mothers and newborns at Regina General Hospital. The interview lasted 60 minutes and was recorded on audio tape. A few weeks following the interview, the interviewee was provided with a verbatim transcript and given the opportunity to amend or withdraw any or all of her responses. The interviewee made no changes. A transcript release form was signed, and the interviewee was allowed to keep a copy of the interview transcript for her personal records. The interview cited to illustrate the mode of questioning used was referenced with a non-identifiable transcript number (Tr.) and its subsequent page number.

Kvale (1996) argues that when active listening occurs for depth interviews, there is variation in the mode of questioning. In his discussion of this topic, he contends that the first few questions should be introducing questions. According to Kvale, introducing questions are opening questions asked to educe the interviewee's understanding of the subjects or phenomena being investigated. In this sense, introductory questions are the stage upon which the interviewer sets up the rest of the interview. If the interview is well designed, the introductory questions provide the opportunity for establishing rapport. In the first exchange cited below, introductory questions are asked regarding the interviewee's current role and her understanding of Parenting Plus: 
Q: Can you describe your role in maternal childcare at Regina General?

A: I provide orientation to new staff that comes on the unit. I develop programs of care for the patients, teaching pamphlets for the patients. I work closely with the co-ordinator to decide what direction our unit is going, where we think the staff needs a little bit more in service, or where they need some help in some areas of interpersonal skills. That's about it I guess.

Q: The next question has to relate to your understanding of the Early Childhood Development program in the Pipestone Health District, Parenting Plus. Could you tell me what is your understanding of Parenting Plus and how it operates in the Pipestone Health District?

A: My understanding is that it's a program that helps families at-risk, parent their children up to age 5. They get involved, first the family is identified through a little referral sheet that we do or some other district may do for them. We identify certain risk factors for them and then the co-ordinator looks at that and decides whether an actual assessment needs to be done, a family assessment needs to be done. (Tr. 8, p. 1)

Upon being asked the second introductory question, the interviewee engages in a rather long description of her understanding of the program and the types of activities that occurred during the home visits. Although the response is amended, it does provide a basis of understanding for the mode of questioning that occurred during the interview session, analysis aside.

The interviewer then asked what Kvale describes as a "specifying question". According to Kvale, the intent is to operationalize the interview by obtaining more precise descriptions.

Q: The next question relates to how you first became involved in the program. Who approached you, what was your understanding of what was requested and your reaction?

A: I believe that it was around November that myself and $K$ got involved. I was approached by $L$, who was approached by $R$. And, $L$ thought I should get involved because we were also looking at a liaison nurse role on our ward. And we were looking at redoing our referral sheet. My understanding at the time was the reason why they really needed us involved was because we 
were kind of going to be the first line, this referral sheet was the first line to help them figure out the risk factors for the family. (Tr. 8, p.1)

As unestablished characters are introduced by the interviewee, the interviewer inquires about $\mathrm{L}$, followed by another query relating to the implementation and development of a screening protocol. The second probe results in the interviewee interrupting prior to completion of the question, indicating her need to express some of the underlying difficulties and apprehensions that surrounded the screening process implemented. Without asking a series of introductory questions that allowed the interviewee to become comfortable and familiar with the interviewer, an indepth understanding of the implementation process might not have been achieved:

\section{Q: Who is $L$ ?}

A: $L$ is my manager, and $R$ is the co-ordinator of the partnership. Really I got involved because part of my role as the educator is to revamp forms and to look at the use of forms and what's necessary on the form. And so then we thought, since we're already looking at changing the form for the liaison role, we might as well take a look at this and see if we can put in some of the risk factors that the Pipestone Health District needed for their optimal child development program.

$\mathrm{Q}$ : And so that didn't prove to be problematic at all? The process involved it...

A: Well it was slightly problematic because some of the risk factors that they had identified, it's a really hard area to get into when you're dealing with the psychosocial aspects of the mother that you have for 2 days. So we only have her in the hospital for 2 days, we don't have a long enough time to really build up a really good relationship with her, a really good rapport with her. So to find out some of these risk factors that they wanted to know, like attempted or unsuccessful abortion for instance, is really. . you need to build up some kind of relationship. So in order for us to put these 10. . they had 10 particular risk scores that they wanted on our form. In order for us to just put that on there, we had a little bit of a difference of opinion. Because I, as a nurse, could see that we weren't going to get that information. Unless it was on her chart, and then we would. . we were very good at going through the history that we have, and especially now that we have the liaison role, we go through the history that we have there and then note anything on the bottom of the form. Any of the information, any of the risk factors that we 
would have noted, would be there. To actually have that box on there, we wondered whether that was a human rights issue, was that any of our business, and my thing as a nurse is, if that was problematic it will play out, she will have symptoms of that being a problem, later on in terms of psychosocial development. If it was problematic, otherwise she's dealt with that. And. . .who am I to bring that back up again? So that was one of the things. The other thing that is a little bit problematic would be the criminal history as well. Criminal history of who? Of the mother? Of the father? Of anybody in the family? My brother was in jail for a week, like what is it all about? And would we then have to go into what was this person in jail for? And if they haven't been tried and convicted you don't really know what's going on there. So then again that's kind of a human rights issue. So we had a bit of a struggle trying to. . " Ok this is what we kind of feel comfortable with on our form. This is what you need. Can you live with this?". . .kind of thing. In the end it came out that we could live with what we had, and they were getting the information that they needed. They could almost tell by our referrals, I think, whether the family would need an assessment. That's basically what they needed. (Tr. 8, p. 3)

The interviewer then asks a follow-up question, reframing the previous question to provide the respondent with an opportunity to discuss what would be a more proactive method by which to conduct screens and referrals for the Pipestone Health District at Regina General Hospital. By listening to what the interviewee had previously said regarding difficulties encountered in the implementation of the screening and referral protocol for Parenting Plus, while at the same time being cognizant of the research questions for which the interviewer is trying to obtain answers, the interviewer is able to elicit further information on the implementation process:

Q: Then can I play "devil's advocate" and ask what would be a more appropriate or user-friendly type of screen for an Early Childhood Development program that would work for Regina General?

A: I think actually that we've developed it now, and we can live with this form that we have. And I think it's actually really user friendly. But it's been through, probably I think. . .4 drafts, since we started. Had we been involved a little quicker-this is my one problem. Had we been involved right from the start, even thinking about this, and had some of the information. (I don't 
think I got this confidential screen and 10 or 12 points that they needed until probably our second draft.) Which would have been December of 1999. And still we hadn't fine-tuned it. We hadn't come to an agreement on what or what shouldn't be on there yet. So had we had a little bit more information maybe about what. . .even some background on the literature search on why they need to know the criminal history. Why do they need to know? What has shown? Why is this a risk factor? The unsuccessful abortion. Why is that a risk factor? That would have maybe been a little bit easier, but I actually think now that we have a form that we both can live with and that we can fashion. Because we're looking at, I call this the optimal, but the Regina Health District is actually looking at the optimal child development program, which is kind of going to be fashioned after the Parenting Plus. We are working towards that so the form that we developed I think is actually going to work really well for the liaison role, for our referral to our public-health nurses and our district nurses, and for the referrals to these optimal child health programs. (Tr. 8, p. 4)

Kvale (1996) refers to the next mode of questioning used by the interviewer as direct questioning. According to Kvale, direct questions are usually asked near the end of the interview, once the interviewee has had the opportunity to respond spontaneously to the previous questions. The purpose behind direct questioning is to introduce new topics or dimensions for discussion (Kvale). In this case, the interviewer asks a direct question, specifically, if threats to the implementation of Parenting Plus have been foreseen.

Q: During the time when discussions were first being initiated with Parenting Plus or the Early Childhood Development Program of the Pipestone Health District and Regina General, were any potential threats to implementation and the design of the program articulated, in terms of some difficulties that might arise?

A: The one difficulty that we thought would be a problem would be to fax, and actually we're not doing that I think. I don't know what we ended up talking about but, and I'm not sure what we're doing right now. But what the difficulty was, was to have to fax this form and this all goes back to the whole consent thing. To fax the form to public health is $O . K$. because we tell everybody when they come into the hospital that I'm filling this out and it's going to go to your public-health nurse in your district. But then to fax the form to the program without that person having a total understanding of the program, without having the brochure and informed consent, we didn't feel 
comfortable doing that. Now I'm not sure where we ended up but in the beginning that was a real concern. Pipestone Health District's concern was that they wouldn't get those forms in a timely manner from their district public health office. And our concern was the actual consent to becoming involved in the program. (Tr. 8, p. 5)

Based on the interviewee's response, the interviewer then asks an interpretive question in which he briefly summarizes and paraphrases the interviewee's response. The purpose behind interpretive questions is to clarify the response of the interviewee:

Q: So the form was sent to the public health office and then Pipestone Health District was requesting that you fax the form as well to Parenting Plus?

A: Right. I don't believe, at the beginning I know we didn't do that, and I don't know what we're doing now. I know now that we have this pamphlet and our liaison nurse goes around and talks to everybody and tells them about our welcome home program and also tells the Pipestone district about their program. Then maybe we fax a form to their office as well. What was happening, was we were faxing it to the district office and then they were then giving it to Pipestone. And actually, I think that's what's still happening because Pipestone was saying something about faxed twice, some of the information. You really have to be careful how you write your information because sometimes it can be illegible by the time it gets to them. (Tr. 8, p. 6)

In light the interviewee's response, the interviewer asks a follow-up question that

pertains to the screening process for First Nations, seeking further elaboration.

Q: What about the First Nations and the federal health nurses? Is it a similar process, in terms of referrals from Regina General?

A: They actually go to the. .I believe they go to the public health office that is looking after that particular district. I don't think that $D$ faxes them to the Band Office. Because we're not really sure. There's a lot of confidential information on here. We're not really sure where that information will end up, in the middle of the Band Office. So I believe she faxes it to the district office and then they get it to their workers. (Tr. 8, p. 6)

The interviewer then changes topics with what Kvale (1996) describes as a structuring question, indicating that the previous theme has been exhausted. In turn, 
the interviewer tries to contextualize the interviewee's response by asking what are his or her primary responsibilities to Parenting Plus:

Q: What do you see as your primary responsibilities, as it relates to Parenting Plus?

A: My primary responsibility is to make sure that the patients are being informed so that they have an informed consent into the program; that our referral works for us and for the Pipestone District; and that we have a good working relationship. I think that ML started out with a gusto and really didn't have a whole lot to start out with because she wasn't really, she was laying new ground as she went along and we didn't have the brochure. We didn't have all the information. So, I believe that now. . I don't remember her name. . the new co-ordinator will have a little easier ground to go on now that the program is kind of under way. It will be a little easier to carry on. (Tr. 8, p. 6)

The dialogue between the interviewer and interviewee continues. Other than nonverbal cues and silence, the modes of questioning developed for this particular sequence reflect a variety of questioning styles to elicit information pertaining to the implementation of Parenting Plus. However, Kvale (1996) cautions, "Active listening - the interviewer's ability to listen actively to what the interviewee says can be more important than the specific mastery of questioning techniques" (p. 132). Thus, by using a variety of questioning styles and engaging in active interviewing by actually listening to what the interviewee is saying, the researcher was able to attend to the layers of meanings and nuances elicited during the semi-structured depth interview. In turn, this allowed the researcher to obtain a breadth and depth of knowledge on a particular subject - in this instance the implementation of Parenting Plus. 


\section{Focus Groups}

As there are advantages and disadvantages with any interview format, the same must be said of group interviews or focus groups. At their worst, focus groups can come to be dominated by one individual, to the detriment of others opinions, or take on their own idiosyncratic group thinking. Because of this, it is important that a trained researcher moderates or directs the group. Nevertheless, according to Fontana and Frey (1994):

The group interview has the advantages of being inexpensive, data rich, flexible, stimulating to respondents, recall aiding, and cumulative and elaborative, over and above individual responses. (p. 365)

Moreover, some researchers have argued that focus groups gather much more data and rich information than would any representative sample (Blumer, 1967).

In planning for focus groups, there are a number of different design options that can be used. A representative, but not exhaustive, list of design options includes: single category designs; multiple-category designs; double-layer designs; and broad involvement designs (Krueger \& Casey, 2000). Initially, a double-layer design was planned for at the August 24, 2000, evaluation meeting with stakeholders. In a double-layer design, it is anticipated that there would be geographical areas (the first layer) from which participants for the focus groups could be drawn (the second layer) (Krueger \& Casey). The advantage of this focus group design is that it allows comparisons and contrasts to be made with different audiences from various locales. However, once program staff began approaching participants with a letter of invitation to the focus group sessions, it was found that the number of willing participants would constitute only a single audience for the focus group. This is the 
traditional type of design where a number of focus groups occur for one audience until theoretical saturation is reached. According to Krueger and Casey, theoretical saturation is achieved when no new understandings or insights are being attained. In using a single-category design, theoretical saturation was achieved through two four-hour focus groups.

Furthermore, because of the potentially personal information that could be disclosed, both practical and ethical limitations were placed on the focus groups. Krueger and Casey (2000) recommend that it is best not to use focus groups when the sharing of information among participants is of a sensitive and personal nature. The alternative to the gathering of such data would be to conduct semi-structured depth interviews with participants. However, program staff argued against this approach because of the perceived difficulty in establishing trust and rapport with participants and subsequent logistics in collecting data (R. Bosman, personal communication, August 24, 2000).

In an effort to reflect the participants' experience of the program, an empowerment evaluation (EE) format was chosen for the focus groups and protocol developed (see Appendix 2). The benefit to conducting EE focus groups was that they provided participants and program staff with an opportunity to evaluate their program in a collaborative, co-operative, and participatory forum, while at the same time creating documentation of the process for later data analysis. However, the impact of EE on Parenting Plus was not determined because it was not implementation focused, and in this sense, did not meet the evaluative needs of the stakeholders. As well, the period of data collection was limited to four months, and 
therefore, any attempt at generating an understanding of $\mathrm{EE}$ on Parenting plus would be incomplete. As a result, the possible cyclical effects of EE were not evaluated. Nevertheless, the data obtained from the focus groups, including artifacts of the program created, could be categorized thematically and analyzed with the general inductive approach.

\section{Empowerment evaluation}

Empowerment evaluation was born out of the ideas and research of Fetterman (1993) surrounding communication, collaboration, and advocacy in program evaluation. Driven largely by Fetterman $(1994,1995,1996,1997,1999)$, EE has become a topic of research debate in North American program evaluation. It is not without its detractors and has been described as an ideological movement rife with dogmatism (Sechrest, 1997). Moreover, Patton (1997b) contends:

This volume [Fetterman et al., 1996] fails to consistently distinguish between participatory and collaborative evaluation processes that may lead to some feelings of empowerment among those involved versus empowerment evaluation as a distinct political process aimed explicitly at and therefore judged by its effectiveness in altering power relationships. (p. 152)

However, Fetterman (1997) argues it is natural that a synergy exists between collaborative, participatory, and empowerment evaluation, and in clarifying the conceptual issues, one should not weaken the bonds connecting these approaches.

Accordingly, the foundational principle that guides EE is the pursuit of truth and honesty which Fetterman's (1999) suggests:

[is]. . .a sincere attempt to understand events in context and from multiple world views. The aim is to try and understand what's going on in a situation from the participants own perspective as accurately and honestly as possible and then proceed to improve it with meaningful goals and strategies and credible documentation. (p. 13) 
The first step in conducting EE involves taking stock and is a process whereby those engaged in evaluating their own program identify its strengths and weakness (Fetterman, 1996, 1997, 1999). According to Fetterman (1999), there is nothing confidential about the data collection process. In fact, he advocates for program participants and staff members to work in an open forum because it allows for discussion:

The taking stock phase of empowerment evaluation is conducted in an open setting for three reasons: 1) it creates a democratic flow of information and exchange of information: 2) it makes it more difficult for managers to retaliate because it is an open forum; 3 ) it increases the probability that the disclosures will be diplomatic because program staff members and participants must remain in that environment. Open discussions in a vacuum, without regard for workplace norms, are not productive. They are often unrealistic and counter productive. (p. 2)

Once participants have rated their program, they are asked what they would like to see happen in the future. This step takes into account the resources, activities, and scope of the program and is directly linked to those areas. Within this process, individuals are called to provide reasons as to why they believe the goals they are advocating for the program are viable and attainable (Fetterman, 1996, 1997, 1999). The third pragmatic step in EE relates to developing strategies for the attainment of goals set in the previous step, which are routinely reviewed to determine their viability (Fetterman, 1996, 1997, 1999). The fourth step in EE involves a documenting process in which participants are asked to identify information that will enable them to monitor the progress of their program in a relevant and timely manner (Fetterman, 1996, 1997, 1999). Thus, based on the EE protocol outlined, the focus groups for the implementation evaluation of Parenting Plus were conducted. 


\section{Family-Centered Program Rating Scale}

A multi-method study benefits from the use of various data sources to answer the same research question. This type of approach to the establishment of validity in qualitative studies is referred to as "triangulation". Both semi-structured depth interviews and empowerment evaluation focus groups are qualitative methods for compiling information and developing understandings. In order to enhance the validity or trustworthiness of the data, quantitative methods can also be used. Therefore, to facilitate the triangulation of data and in order to further elaborate on the research questions posited, the Family-Centered Program Rating Scale (FamPRS) (Murphy, et al., 1991) was administered to participating parents.

According to Murphy, Lee, Turnbull and Turbiville (1995), the FamPRS is a paper-and-pencil instrument designed for the evaluation of early intervention programs. It is used widely and the benefits in using the instrument to evaluate early intervention and early childhood service providers' efforts have been reported (Hammond, 1999). Because one of the intents behind its development was to "use parents' perspectives as the standards for judging a program's performance" (Murphy et al., 1995, p. 33), it was of particular interest to this study as it provided a means by which data were triangulated. Murphy et al. (1995) state that the instrument is based on family-centered practices as defined by the Beach Center on Families and Disabilities, where it was developed. According to Murphy et al., family-centered practices:

(a) include families in decision-making, planning, assessment, and service delivery at family, agency, and system levels;

(b) develop services for the whole family and not just the child;

(c) are guided by families' priorities for goals and services; and 
(d) offer and respect families' choices regarding the level of their participation. (p. 25)

In completing the FamPRS, respondents use a 4-point likert scale to rate each item twice on the instrument - once for how they perceived their program was doing on each item and once for how important the item was to them personally. Consequently, importance and performance scores are collected for the 11 subscales. Lee (1993) tells us that inferences derived from the instrument include:

1. Inferences about parents' and staff members' perceptions of their programs;

2. Inferences about comparisons between programs;

3. Inferences about the effectiveness of staff development and program modification efforts;

4. Inferences about long-term and short-term outcomes for children and families; and

5. Inferences about the effects of using the FamPRS on programs, families and communities. (p. 34)

In depicting how the FamPRS was developed and its psychometric properties, Murphy et al. (1995) describe the development of the revised instrument that includes 59 items for 11 subscales. The data analysis reported by Murphy et al. involved principal components extraction with varimax rotation with item loadings $<.40$ on all factors resulting in elimination. Subsequently, 12 factors were identified with the Kaiser criterion and then supported through a scree test. Moreover, the factors derived accounted for $63.2 \%$ of the total scale variance (Murphy et al.). A 12 factor solution was originally obtained and the resultant 11 factor solution came about with the combination of the two statistically independent subscales into one, entitled Providing Appropriate and Practical Information, because of conceptual and functional relatedness of the two factors (Murphy et al.). The authors state that, for every stage of development, expert judgment was sought from parents, national 
leaders, program administrators, teachers, therapists, and paraprofessionals.

Furthermore, internal consistency coefficients of subscales for both parents and staff members are reported by Murphy et al. as a mean coefficient alpha of .79 for parents and a mean coefficient alpha of .74 for staff. Thus, the subscales derived from the 11 factor loading include:

1. Flexibility and innovation in programming;

2. Providing and coordinating responsive services;

3. Individualizing services and ways of handling complaints;

4. Providing appropriate and timely information;

5. Communication and timing;

6. Developing and maintaining comfortable relationships;

7. Building family-staff collaboration;

8. Respecting the family as decision-maker;

9. Respecting the family's expertise and areas of strength;

10. Recognizing the family's needs for autonomy; and

11. Building positive expectations. (p. 34-35)

To ascertain the suitability of the FamPRS for the implementation evaluation, the instrument was piloted at the second focus group held with participating parents. At that session, parents reviewed the FamPRS and voiced concern about items in the instrument that spoke of child disability and were viewed as pejorative (Participant Focus Group, personal communication, November 21, 2000). Based on the feedback from the parents, permission to adapt the FamPRS was requested and received from Anne P. Turnbull (personal communication December 4, 2000), researcher on the instrument and director for the Beach Centre on Families and Disabilities at the University of Kansas where it was developed. This resulted in the creation of an adapted version of the FamPRS parent form (see Appendix 3) with the wording on seven items changed from the original (see Table 3.5). 
Table 3.5.

Original and Adapted Items of the FamPRS Parent's Scale

\section{Original Items}

8. In this program the IEP, or IFSP (Individualized Family Service Plan), is used as a "plan of action".

11. The program gives other children in my family support and information about their brother's or sister's disability.

17. Staff members help my family learn how to teach our child special skills.

27. Staff members give my family clear and complete information about our child's disability.

28. Staff members tell my family what they have learned right after our child's evaluation.

48. Staff members ask my family's opinions and include us in the process of evaluating our child.

59. My family is an important part of the team when our IEP, or IFSP (Individualized Family Service Plan), is developed, reviewed, or changed.

\section{Adapted Items}

8. In this program goal setting is used as a "plan of action".

11. The program gives the other children in my family support and information about their newborn brother or sister.

17. Staff members help my family learn how to teach our child different skills.

27. Staff members give my family clear and complete information about our child.

28. Staff members tell my family what they have learned about our child and family.

48. Staff members ask my family's opinions and include us in the process of understanding our child.

59. My family is an important part of the team when goal setting is developed, reviewed, or changed.

\section{Document Review}

The purpose behind the document review conducted for Parenting Plus was to determine key items that included descriptive statistics of program participants and program delivery rates. Information for the document review was obtained through Parenting Plus work plans, reports, home visiting protocol, family support plans, home-visitation records, screening protocol, referral, and assessment forms. From a utilization-focused perspective, the gathering of service delivery rates is important for the evaluation as it provides a means by which management can monitor the implementation of the program. 


\section{Data Collection}

As the study involved research with human subjects, informed consent was required. An application to the University of Saskatchewan Advisory Committee on Ethics in Behavioral Science Research made on June 1, 2000, received final approval on August 31, 2000 (see Appendix 4). To enable data collection for the evaluation of Parenting Plus, both interview and focus group consent forms, as well as letters of invitation and data transcript release forms, were developed (see Appendix 5). Data collection occurred during the period September 2000 to December 2000. The methods of data collection employed included:

1) Semi-structured depth interviews of major stakeholders and program staff;

2) Focus groups with program participants;

3) Parent's scale of the Family-Centered Program Rating Scale adapted for Parenting Plus; and

4) Document review.

\section{Interview Data Collection}

The questions developed for the interview protocol are derived from the research literature surrounding home visitations, Patton's (1997a) menu for sample program implementation questions, and discussions with Parenting Plus stakeholders. The protocol explores feasibility and compliancy issues, as well as formative and summative implementation concerns that include factors related to the delivery of the early childhood development services provided. Subsequently, four open-ended semi-structured depth interview protocols were developed for the Parenting Plus evaluation (see Appendix 6). The rationale for the development of four separate open-ended protocols is that each would reflect questions pertaining to 
the role played by stakeholders in the implementation process. Thus, protocols were created for the director of population health for the Pipestone Health District, the Parenting Plus program co-ordinator, the Parenting Plus FSWs, and other relevant stakeholders. Similar themes pertaining to the implementation process relevant to the role the interviewee played are explored in each interview protocol.

Purposeful sampling (Creswell, 1998) was used to gain insight and understanding inherent in the relevant knowledge base of the different stakeholders. Furthermore, because program staff had a more in-depth knowledge and understanding of Parenting Plus, two interviews were scheduled. This, in turn, allowed the researcher ample time to review the original transcript and determine what new or remaining questions needed to be asked at the next interview so that maximum saturation would be achieved. In-person interviews with Parenting Plus program staff included the director of population health for the Pipestone Health District, the Parenting Plus program co-ordinator, and the two family support workers. The interviews occurred at the Grenfell Health Center in Grenfell where Parenting Plus's program offices are located, during September and December 2000.

A slightly different data collection process occurred for the other major stakeholders participating in the study. As they were mainly health-care professionals with demanding roles and responsibilities, inquiries were made by the researcher regarding their availability for the study. Of those that agreed to participate, the length of the dialogue was one of the most common concerns. Therefore, it was decided in consultation with stakeholders to limit the interviews to 
one, lasting up to 60 minutes in length. This allowed for a number of conversations to be conducted with stakeholders. As well, owing to the geographical diversity of the region and distance between major stakeholders, discussions were conducted over the telephone when in person interviews were not possible. Although, telephone interviews are often criticized as a means to solicit depth responses owing to their impersonal nature (Shuy, 2002), the researcher did not find this observation to be true. In fact, stakeholders valued their opportunity to participate because they perceived it as an occasion for their voices to be heard on a topic in which they had an intense interest.

The stakeholders were chosen to participate in the interview process on the basis of their role in the development of the program, their current role on the Parenting Plus advisory council, or their interest in early intervention within the district. The - interviews explored the roles and experiences of the various stakeholders and program staff in project development and the processes involved in Parenting Plus's implementation. In using a general inductive approach to data analysis, the researcher was able to determine an understanding of the characteristics that hindered or facilitated the program's implementation and the model of service delivery that has emerged.

Stakeholders interviewed in person included four staff from Regina General Hospital involved in the screening and referral process; a local physician; the former CEO for the Pipestone Health District; and the director and research officer from the SIPH. Stakeholders interviewed over the telephone included the six public-health nurses from the Pipestone Health District, two community-health nurses from First 
Nation Health Centers, the Early Childhood Intervention Program co-ordinator in the Pipestone Health District, the former director of population health for the Pipestone Health District, and the marketing representative for the corporate sponsor SaskTel. In total, over twenty-three individuals were interviewed using semi-structured depth interviews for the implementation evaluation of Parenting Plus (see Table 3.6).

Payne (1999) describes two types of strategies that can be employed to establish the validity or trustworthiness of qualitative interviews, including respondent validation and triangulation. For the present study, respondent validation of the original transcripts occurred, as well as the opportunity for review and feedback of the emerging themes and summary findings by the interview participants. All participants received verbatim transcripts of their interviews for assessment. Moreover, a formative report of emerging findings and a draft summary report was made available to Parenting Plus and distributed to stakeholders. As well, trustworthiness of the data collected is enhanced through use of the general inductive approach where it is advocated that between 20-30 interviews be conducted (Thomas, 2000). Thus, to aid in establishing trustworthiness of the interview data, 23 semi-structured depth interviews with stakeholders occurred. Moreover, to ensure the data was auditable while guaranteeing anonymity, as articulated on the interview consent form signed by participants, interview quotations cited were referenced with a non-identifiable transcript number (Tr) and page number, the coding for which is stored by the primary investigator with the transcripts and audio tapes of the interviews. 
Table 3.6

\section{Interview Data Collection Schedule}

\section{Contact Type of Interview Date of Interview}

\begin{tabular}{|c|c|c|c|c|}
\hline & In-Person & Telephone & Interview 1 & Interview 2 \\
\hline Parenting Plus Program Co-ordinator & $\mathrm{X}$ & & $9 / 15 / 2000$ & $12 / 01 / 2000$ \\
\hline Parenting Plus, F SW & $\bar{X}$ & & $9 / 15 / 2000$ & $12 / 01 / 2000$ \\
\hline Parenting Plus, F SW & $\mathrm{X}$ & & $9 / 15 / 2000$ & $12 / 01 / 2000$ \\
\hline $\begin{array}{l}\text { Pipestone Health District } \\
\text { director of Population Health }\end{array}$ & $\mathrm{X}$ & & $9 / 15 / 2000$ & $12 / 01 / 2000$ \\
\hline $\begin{array}{l}\text { Regina General Hospital } \\
\text { home program liaison }\end{array}$ & $\mathrm{X}$ & & $9 / 11 / 2000$ & \\
\hline $\begin{array}{l}\text { Regina General Hospital } \\
\text { social worker NICU }\end{array}$ & $\bar{X}$ & & $9 / 11 / 2000$ & \\
\hline $\begin{array}{l}\text { Regina General Hospital } \\
\text { clinical development educator }\end{array}$ & $\bar{X}$ & & $9 / 11 / 2000$ & \\
\hline $\begin{array}{l}\text { Regina General Hospital } \\
\text { maternity unit co-ordinator }\end{array}$ & $\mathrm{X}$ & & $9 / 11 / 2000$ & \\
\hline $\begin{array}{l}\text { Pipestone Health District } \\
\text { former CEO }\end{array}$ & $\bar{X}$ & & $9 / 25 / 2000$ & \\
\hline $\begin{array}{l}\text { Pipestone Health District } \\
\text { local physician }\end{array}$ & $\mathrm{X}$ & & $12 / 01 / 2000$ & \\
\hline SIPH, director & $\mathrm{X}$ & & $12 / 06 / 2000$ & \\
\hline SIPH, research officer & $\mathrm{X}$ & & $12 / 06 / 2000$ & \\
\hline SaskTel, marketing director & & $\mathrm{X}$ & $11 / 14 / 2000$ & \\
\hline $\begin{array}{l}\text { Pipestone Health District } \\
\text { community-health nurse }\end{array}$ & & $\mathrm{X}$ & $12 / 05 / 2000$ & \\
\hline $\begin{array}{l}\text { Pipestone Health District } \\
\text { community-health nurse }\end{array}$ & & $\bar{X}$ & $12 / 06 / 2000$ & \\
\hline $\begin{array}{l}\text { Pipestone Health District } \\
\text { ECIP, executive director }\end{array}$ & & $\mathrm{X}$ & $12 / 06 / 2000$ & \\
\hline $\begin{array}{l}\text { Pipestone Health District } \\
\text { PHN }\end{array}$ & & $\mathrm{X}$ & $12 / 07 / 2000$ & \\
\hline $\begin{array}{l}\text { Pipestone Health District } \\
\text { PHN }\end{array}$ & & $\mathrm{X}$ & $12 / 07 / 2000$ & \\
\hline $\begin{array}{l}\text { Pipestone Health District } \\
\text { P H N }\end{array}$ & & $\mathrm{X}$ & $12 / 08 / 2000$ & \\
\hline $\begin{array}{l}\text { Pipestone Health District } \\
\text { PHN }\end{array}$ & & $\mathrm{X}$ & $12 / 08 / 2000$ & \\
\hline $\begin{array}{l}\text { Pipestone Health District } \\
\text { PHN }\end{array}$ & & $\mathrm{X}$ & $12 / 11 / 2000$ & \\
\hline $\begin{array}{l}\text { Pipestone Health District } \\
\text { PHN }\end{array}$ & & $\mathrm{X}$ & $12 / 14 / 2000$ & \\
\hline $\begin{array}{l}\text { Pipestone Health District } \\
\text { former director Population Health }\end{array}$ & & $\mathrm{X}$ & $12 / 12 / 2000$ & \\
\hline
\end{tabular}




\section{Focus Group Data Collection}

All parents participating in the program were invited to attend the focus groups. Letters of invitation were either mailed or delivered in person by the FSW. Thus, two focus groups, with six people in attendance at each, were established from participating parents and the FSW. This type of sampling for either qualitative or quantitative research is referred to as convenience sampling as it is done on the basis of subject availability (Miles \& Huberman, 1994; Tashakkori \& Teddlie; 1998). The focus groups were held at Broadview Hospital on October 24 and November 21, 2000

Although the data collected were of a less personal nature, it was still important that ethical considerations regarding the information obtained by the researcher be addressed. As participants shared understandings, insights, and experiences of the program with other participants during the focus groups, it was necessary that they be aware of their obligation to respect the privacy of other members of the group by not disclosing any shared personal information. As a result, a consent form that addressed ethical issues and the rights and responsibilities of focus group participants was developed (see Appendix 5). The consent form was explained and read through at the focus groups by the principal investigator and signed by those willing to participate in the session.

The focus groups resulted in the creation of a mission statement for the program, the listing and prioritizing of the program's key activities as performed during home visitations, and the establishment of program goals. Once the goals based on the key activities were established, the principal investigator elicited strategies for the 
attainment of the goals and the documentation required to determine if the goals were obtained.

The attainment of the focus group empowerment evaluation documentation occurred through a four-phase writing process that involved brainstorming, drafting, revising, and finalizing. The brainstorming and drafting phases characterized the first focus group, while revising and finalizing distinguished the second. As well, the second focus group was used to pilot and solicit feedback regarding the FamPRS (Murphy et al., 1991) which was used as a quantitative measure for the implementation evaluation of Parenting Plus.

\section{FamPRS Data Collection}

Due to the transient nature of the families involved in Parenting Plus and potential difficulties in contacting parents and having the FamPRS completed and returned, it was decided not to conduct a home mail out of the instrument. Rather, the FSW sought verbal consent from the parents to complete the FamPRS when the FSW conducted a home visit during December, 2000. Of the 18 parents participating in the program, 11 FamPRS were completed and returned to the principal investigator during the week ending December 24, 2000.

\section{Document Review Data Collection}

Data collection for the document review occurred over a four-month period from September to December, 2000. Release of information was granted by the program co-ordinator for the collecting of data pertaining to service delivery rates 
and descriptive statistics of program participants. Data for the document review came from Parenting Plus work plans, reports, family support plans, home-visitation records, screening protocol, referral, and assessment forms.

\section{Data Analysis}

The qualitative data collected for the semi-structured interviews and focus groups were analyzed using inductive analysis as recommended by Patton (1990) and Thomas (2000). The analysis involved organizing raw data from interview transcripts and focus group document artifacts into meaningful themes and categories (Patton, 1990; Thomas, 2000). The data analysis process involves clustering quotations around units of meaning and underlying categories until themes emerge. According to Thomas, in creating the categories, two general rules should be applied. The rules pertain to overlapping coding and uncoded text and include:

1. A single text segment may go into more than one category; and

2. A considerable amount of text may not be assigned to any category, as much of the text may not be relevant to the research objectives. (p. 8).

Thus, the analysis was deemed complete when it was not possible to determine further underlying categories to create higher order themes. The procedure for data analysis in the study included the following six steps:

1. The tapes were initially listened to by the researcher; the transcripts were read and reread until the researcher was fully familiar with the content;

2. Raw data categories were identified in the form of quotations or paraphrases;

3. Each raw data category was transferred to separate sheets for further data 
analysis;

4. General inductive analysis was conducted to identify common themes of greater generality from the raw data categories (identified in step 2). Higher level themes were labeled first order or second order themes, and the highest level was labeled as a general dimension (those of greatest abstraction);

5. The researcher's individual biases were controlled for by participant review of the evolving categories and themes; and

6. The researcher to provide a validity check conducted deductive analysis. In this respect, the researcher reread the transcripts to verify that all themes and categories were represented.

Data analysis for the document review involved the determination of descriptive statistics of the screening and assessment protocol. In this respect, the base rates for screening and assessment, the demographic characteristics of the participating parents, and indicators of service delivery were calculated. The descriptive statistics for the screening and assessment protocol included: frequency counts and percentages for the type of screen (prenatal or postnatal); status of screen (positive, negative, or unknown); and percentages for risk factors identified on the assessment protocol, the Family Stress Checklist (Kempe, 1976). Demographic descriptive statistics for participating parents included frequency counts based on maternal age of mother, marital status, education level, and employment. Service delivery rates abstracted included frequency counts for the number of families in the program, the length of time families were participating, frequency of home visitations conducted by the paraprofessionals, frequency of telephone contacts made by the 
paraprofessionals, and frequency of program levels for participating families. The formulas used to determine the base rates for screening, assessment, and number of participating families were those prescribed by the Hawaii Healthy Start model. Based on this model, the base rates for Parenting Plus were determined through the application of the following formulas (Hawaii Department of Health, Maternal and Child Health Branch, 1994b):

1. Number of live births $\times 90 \%=$ total number expected to be screened.

2. Total number screened $\times 20 \%=$ total number of families identified for family assessment.

3. Total number of families identified for family assessment $\times 90 \%=$ total number of families accepting services.

4. Total number of families accepting services $\times 20 \%=$ Annual attrition rate of families. (p. 72)

Data analysis for the FamPRS followed the instructions provided in the users' manual. On individual forms completed by parents, the performance and importance scores were determined for each item. The scores for the subscales were calculated by first converting the ratings of the respondents to numbers. These were then transferred to the item tally form, where the total number of individual responses for each item were entered so that a group average could be calculated. Once the performance and importance averages for each item were calculated for all respondents, the scores were transferred onto a group scoring form. On the group scoring form, the average scores for each item in the performance and importance domains were scored for each subscale so that an average performance and importance score was determined for each of the 11 subscales on the FamPRS. Once the group scoring form was completed, the performance and importance scores were transferred to a program profile form so that patterns in the results could be derived. 


\section{Summary}

Program evaluation is an applied area of early childhood development. The implementation evaluation of Parenting Plus was no exception. It was an external evaluation that provided internal formal participation by evaluation stakeholders in objectifying the evaluation and its design. The stakeholder evaluation meetings for the program began three months after start-up and continued for the first year of the program. During this time, a mixed-methods implementation evaluation framework was designed that used the general inductive approach to qualitative data analysis and quantitative descriptive statistics of service delivery rates, as well as parentcompleted early childhood development program evaluation instrumentation.

The objectives of the evaluation were, first, to understand the fidelity of program implementation to the program model, Hawaii Healthy Start, and the program model operationalized. Second, to determine the characteristics of the process that either facilitated or hindered the implementation of Parenting Plus. Finally, the third objective was to gain an understanding of the participants' experiences of Parenting Plus. The summary findings then result in a conceptual model depicting the operationalization of the program. Thus, by gaining insight and understanding into the implementation of Parenting Plus, similar efforts may benefit.

Ultimately, the value of any research, in post positivist terms, lies in its ability to be valid and reliable. These terms are commonly found within the literature surrounding quantitative research. From this perspective, internal and external validity, reliability, and objectivity are the standards by which a study is judged. In a 
constructivist sense, these terms qualitatively are embraced as "trustworthiness" and "authenticity". Even though the present study is a mixed-methods design, it is clear from the discussion so far that the qualitative methods of the study account for a substantial portion of the information. As a result, it is necessary to engage in a discussion surrounding the trustworthiness of the data.

Throughout, elements of good practice have been articulated. Foremost to the dialogue is the intensive engagement by the researcher with the material for a prolonged period enabling a depth and breadth of knowledge surrounding the implementation of Parenting Plus. As well, dependability has been evidenced by member checking with the participants in the interviews and focus groups. Within the context of the interviews, authenticity was further enhanced through active listening and by using a variety of questioning styles. Through formal and informal member checks of stakeholders participating in the interviews and focus groups, the researcher was able to determine if the constructs or emerging themes were representative of their perceptions and experiences. Triangulation among data sources including interviews, focus groups, document review, and instrumentation also insured reliability. Finally, trustworthiness would not have been achieved without a continual process of peer review that came to be embraced within the context of the dissertation committee. 


\section{CHAPTER FOUR}

\section{Results}

\section{Overview}

As Parenting Plus is a pilot program, stakeholders decided to direct evaluation efforts towards process as opposed to outcome variables. A utilization-focused implementation evaluation framework that included both quantitative and qualitative methods was designed. In operationalizing the evaluation, three separate but interrelated objectives were outlined. The first was to compare how Parenting Plus as implemented compares to the program model, Hawaii Healthy Start. The second was to determine the characteristics of the process that either facilitated or hindered the implementation of Parenting Plus. The third was to solicit the participants' experience and perspective in determining the system of service delivery that evolved and is being applied. The general inductive approach was used for qualitative data analysis, and descriptive statistics for service delivery rates and a parent-completed early childhood development program evaluation instrument are reported.

In reviewing Hawaii Healthy Start, challenges to the implementation of program protocol have resulted in significant changes and modifications to the program over the past decade (Duggan, McFarlane et al., 1999; Duggan, Windham et al., 2000). For example, in a review of the literature conducted by Duggan, McFarlane et al. on randomized trials of Hawaii Healthy Start, only one third of the programs provided the number of visits advocated by the protocol. Second, in conducting process evaluations of 
home visitations, Duggan, McFarlane et al. advocate for future research endeavors to consider the process not only from the perspective of the service provider but also from the families. Third, local values and needs must be considered in relation to how they effect program implementation (Duggan, McFarlane et al.). Thus, it would be ill-advised to adopt a home-visiting program model simply because it has had success in other settings without considering the context in which it is to be implemented.

In a qualitative case study on the implementation of an early intervention program conducted by Britt (1998), it was reported that, when resources are constrained, it is difficult for program staff to follow the mandate of the program as addressed by its protocol. This illustrates how resource allocation during the implementation phase of a program can affect service delivery. Consequently, if resources are constrained, programs can become reactive crisis interventions, as opposed to proactive strength-based modes of helping. Moreover, the early intervention research literature clearly identifies the need to personalize program theory, goals, structures, and processes for the individual client. Through an understanding of the processes involved, early intervention programs are better able to maximize the quality of treatment effects of the services they provide (Blair \& Ramey, 1997; Britt; Kitzman, Olds, et al., 1997; Kitzman, Yoos, et al., 1997; Ramey \& Ramey, 1993). These conclusions stimulated the following research questions concerning the implementation evaluation of Parenting Plus. 


\section{Research Questions}

\section{Research Question 1}

As Parenting Plus has been implemented, what is the model of service delivery that has emerged and been operationalized?

\section{Research Question 2}

What characteristics of the implementation process for Parenting Plus have facilitated or hindered the systematic screening of high-risk families of newborns, the provision of paraprofessional home visitations for those families, the development of informal community supports for families, and the mechanisms for the co-ordination of supports and services?

\section{Research Question 3}

What do the participants' experience and what do they understand about the paraprofessional home visitations they receive from Parenting Plus?

\section{Research Question Results}

\section{Program Status}

One of the purposes behind an implementation evaluation is to document and establish a record of development for program monitoring purposes (Patton, 1997a). Moreover, recent evaluation studies of Hawaii Healthy Start point to a continued need to report program characteristics as indicators of service delivery that effect outcomes (Duggan, McFarlane et al., 1999; Duggan, Windham et al., 2000). For the present study, 
the results obtained from the document review provide data as it relates to the model of service delivery that has emerged and been operationalized. Therefore, descriptive characteristics of the screening and assessment protocol are summarized and presented to make clear who is being served by Parenting Plus. As well, indicators of service delivery that include base rates for screening and assessment; frequency and length of paraprofessional home visitations; and number of attempted home visitations are abstracted. The document review provides a quantitative description of the program's status and service delivery. As an implementation evaluation, the results provide quantitative descriptive features of the program that can form baseline indicators for future endeavors.

According to the document review conducted in the fall of 2000, Parenting Plus is serving 18 families in the Pipestone Health District and has discharged eight families for a total of 26 families that have received services since the program's inception in September 1999. Moreover, in its first year of operation, Parenting Plus has received 136 screens of which $9 \%$ were prenatal and $91 \%$ were postnatal (see Table 4.1 ). Of the total

Table 4.1

Parenting Plus Screening Tracking Summary

\begin{tabular}{lcc} 
Type of Screen & Frequency & Percentage \\
\hline Prenatal Screens & 12 & 9 \\
Postnatal Screens & $\underline{124}$ & $\frac{91}{100}$ \\
Total Screens & 136 & 100
\end{tabular}

Source: Document Review, October 2000.

number of screens, $48 \%$ were negative, $30 \%$ were positive, and $21 \%$ had seven or more unknowns (see Table 4.2). As Parenting Plus follows a two-stage screening and 
assessment protocol, all screens that are scored as positive or with seven or more unknowns were designated for further assessment with the Family Stress Checklist (Kempe, 1976).

Table 4.2

Parenting Plus Status of Screen

\begin{tabular}{lcc} 
& Frequency & $\underline{\text { Percentage }}$ \\
\cline { 2 - 2 } Negative Screens & 66 & 48 \\
Positive Screens & 41 & 30 \\
7 or more Unknown & 29 & 21 \\
Missing & $\underline{0}$ & $\underline{0}$ \\
Total screens & 136 & 100 \\
\hline
\end{tabular}

Source: Document Review, October 2000.

In determining the base rates for the screening and assessment protocol used for Parenting Plus, the population and staffing projections provided in the Hawaii Healthy Start manual (Hawaii Department of Health, Maternal and Child Health Branch, 1994b) were consulted. According to the Hawaii Healthy Start manual, $90 \%$ of live births will receive screening for any given health district in which the program is in operation. Furthermore, of the total number screened, $20 \%$ will be identified at psychosocial risk with the Family Stress Checklist (Kempe, 1976) and offered the program. Of the number of families identified as overburdened, $90 \%$ will accept services at intake and through an annual attrition rate, $20 \%$ of the families accepting services at intake will drop out. The remaining $80 \%$ of the families represent the total number of families served during the year. In turn, one FSW is required for the program for every 15 families served. The formulas used for the base rates are expressed as follows (Hawaii Department of Health, Maternal and Child Health Branch): 
1. Number of live births $\times 90 \%=$ the total number expected to be screened.

2. Total number screened $\times 20 \%=$ total number of families identified for family assessment.

3. Total number of families identified for family assessment $\times 90 \%=$ Total number of families accepting services.

4. Total number of families accepting services $\times 20 \%=$ Annual attrition rate of families. (p. 72)

One of the difficulties in determining the number of families to be served by Parenting Plus begins with the number of live births. As most of the babies in the health region are born in hospitals outside of the district, the calculation of the total number screened is not as straightforward as it would appear. To aid in this calculation, population data that represent the number of Saskatchewan residents by age and sex in a given health district were obtained (Saskatchewan Health, 2000). From the 1997 data reported by Saskatchewan Health, it was found there are 218 children under one year of age in the Pipestone Health District, 105 boys and 113 girls. If this number is used as an indicator of the number of live births in the region for one year, then the total number screened for the program should be higher than $136(90 \%$ x $218=196)$. However, the population data consulted indicate that the number represented might overestimate the number of First Nation people living on their reserves. This is significant to the screening efforts for Parenting Plus because there are five First Nation communities in the Pipestone Health District. Although there is an apparent discrepancy, the number of families screened positive, accepting assessment, and in the program is what would be expected when the Hawaii Healthy Start population and staffing formulas are used.

Of those families screened that required further assessment, $70 \%$ accepted assessment, $20 \%$ did not accept assessment, and 10\% could not be contacted. To be offered home-visitation services, a positive assessment - a score of 25 or greater for either 
parent on the Family Stress Checklist - had to be obtained. Of the total number that were screened, $60 \%$ assessed positive and accepted the program, 32\% had a negative assessment, and $8 \%$ refused the program. When the Hawaii Healthy Start base rates are compared to those of Parenting Plus, it is found that Parenting Plus had a slightly higher number of families identified at psychosocial risk and accepting services than the Hawaii Healthy Start model (see Figure 4.1).

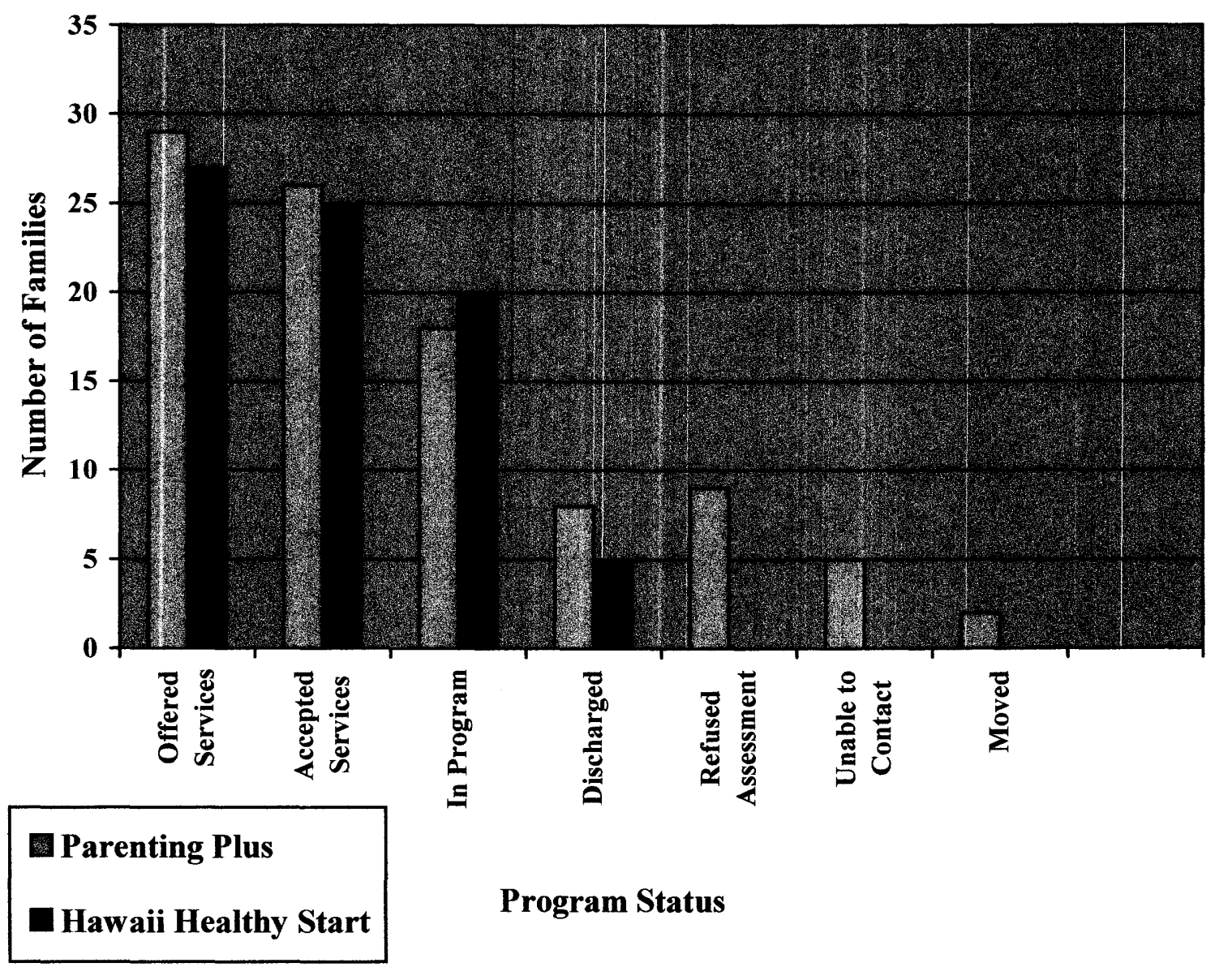

Figure 4.1. Parenting Plus Program Status: October 2000.

Although it would appear from the results that fewer families are enrolled in the program and more are being discharged, in compared with the Hawaii Healthy Start 
model, this conclusion would be inaccurate because at the time of the document review, two families had recently left the program decreasing the number of participants. The departure of the participants was due to their moving out of the health district and did not reflect on the services being provided by Parenting Plus (R. Bosman, personal communication, October 4, 2000). Moreover, it was not unexpected that attrition would occur and it was anticipated that two new families would be enrolling in the program. Based on these findings, Parenting Plus was close to being on target in its goal to deliver systematic screening and assessment of high-risk families of newborns in its first year of operation.

To develop an understanding of the characteristics of the implementation process and operationalization of Parenting Plus, descriptive statistics of the participants in the program are reported. This allows a better understanding of who was being served. The results reported are abstracted from the medical record screen and Family Stress Checklist (Kempe, 1976) used by Parenting Plus. Descriptive statistics only of the mother are reported, although information during a personal interview with the birth mother using the Family Stress Checklist (Kempe) might report on the father of the child from the mother's perspective, little demographic data on the father or other significant male was available from the document review. Thus, only the demographic characteristics for the birth mothers enrolled in the program are reported as frequency distributions and percentages of the total (see Table 4.3). As a result, the demographic characteristics abstracted include the age of mother, marital status, education level of mother, and parental employment. 
The descriptive statistics reveal that over two thirds of the women who received home-visiting services through Parenting Plus were single. This is not surprising because being single is one of the three items that immediately flags a parent as receiving a positive medical screen and being offered further family assessment. The other two items were unsuccessfully sought or attempted abortion and inadequate prenatal care. What these results indicate is that Parenting Plus mainly serves single mothers who are overburdened and that married parents are underrepresented.

To aid in determining who the program is serving and how it has been operationalized, the participants are identified by age group: those in their teenage years, those in their early twenties, those in their mid to late twenties, and those over thirty years of age. Based on these categories, the results indicate that Parenting Plus participants are either in their teens or early twenties and that the number of parent participants tends to drop off as their ages increase. Thus, younger parents, especially those who are single and teenagers, are more likely to be identified as overburdened and to see benefit in participating in a program like Parenting Plus. However, Parenting Plus also has one parent participant over the age of forty and one over the age of thirty. Whether this is unique to the program or a trend that would develop in a scaled-up version requires further research into the demographic composition of home-visiting programs. In terms of education, most of the mothers participating in the program have less than Grade 12, and a few are still enrolled in high school. As for employment status, it is found that the majority of mothers are unemployed. In summary, the results from the demographic profile indicate that the majority of mothers served by Parenting Plus are single, unemployed, and have less than a Grade 12 education. Over a third of the total number of 
participants are teenagers, and over half of the total number of participants are under 25 years of age. This demographic profile is characteristic of mothers who are identified as overburdened and enrolled in the program.

Table 4.3

Participant Demographic Profile

Frequency $\quad \%$

$\begin{array}{llcc} & 16-19 \text { years } & 7 & 39 \\ \text { Age } & 20-23 \text { years } & 5 & 27 \\ & 24-27 \text { years* } & 3 & 17 \\ & >30 & \underline{2} & \underline{17} \\ \text { Marital } & \text { Single } & 18 & 100 \\ \text { Status } & \text { Common Law } & 4 & 72 \\ & \text { Married } & \underline{1} & \underline{6} \\ & & 18 & \underline{6} \\ \text { Employment } & \text { Employed } & 4 & 100 \\ & \text { Unemployed } & \underline{14} & 22 \\ & & 18 & \underline{78} \\ \text { Education } & <\text { Grade } 12 & 16 & \\ & >\text { Grade } 12 & \underline{2} & \underline{17} \\ & & 18 & 100\end{array}$

* there were no participants 28 and 29 years of age.

Source: Parenting Plus document review October 2000.

To help determine how Parenting Plus has been operationalized, in regard to the screening and assessment process, frequency counts for risk factors expressed as a percentage were abstracted from the Family Stress Checklist. In total, data were collected from 18 Family Stress Checklists. In all cases, the birth mother was present for the interview with Parenting Plus program staff. The information obtained for the completion of the Family Stress Checklist was reported by the birth mother as in only one case was 
the birth father present (R. Bosman, personal communication, October, 4, 2000). Results obtained from the Family Stress Checklist indicate that, in one third of the cases, the four risk factors for the mother that were rated most frequently as severe include: parent abused as a child; either one or more of criminal history, mental illness, and substance abuse; multiple life stressors; and either one or both of low self-esteem and poor coping skills (see Figure 4.2). As well, over $75 \%$ of the mothers were not suspected of previously abusing a child.

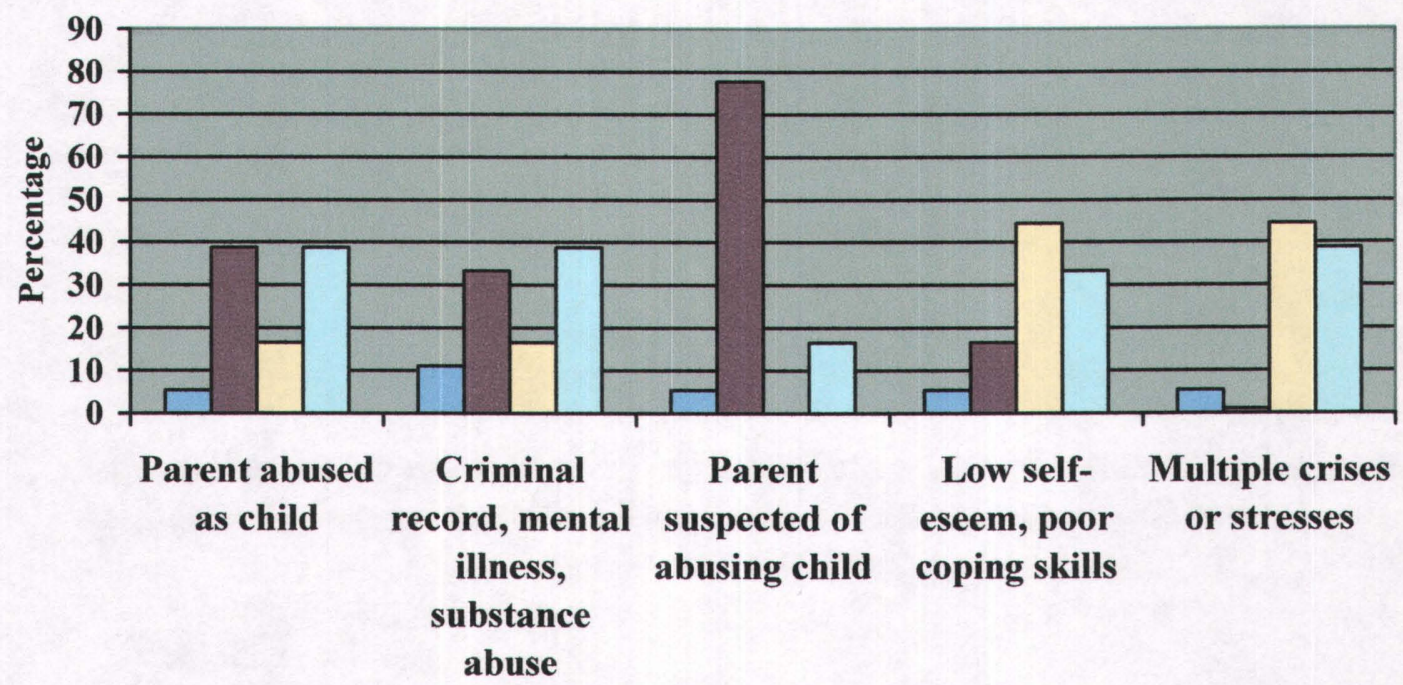

\section{$\square$ Unknown $\square$ No Problem $\square$ Mild Problem $\square$ Severe Problem}

Figure 4.2. Birth Mother Family Stress Checklist Score: Items 1-5

Risk factors for the mother that were rated as mild in over one half of the cases included unrealistic expectations of the child's development and child unwanted or at poor risk of bonding (see Figure 4.3). Risk factors that did not rate as a problem in over half of the cases included: previous or current child protection services; potential for violent temper outbursts; and harsh punishment of the child. 
When examining the risk factors that are present for the fathers, what is of interest is the frequency of unknowns that are reported. This may indicate a lack of involvement on the part of the father in either the assessment process, the family unit, or both. However, of those risk factors that figured predominately for the fathers, it was found that parent abused as child; either one or more of criminal history, mental illness, and substance abuse; and multiple life stressors were most predominant (see Figure 4.4). Except for low self-esteem, these results parallel those found for the mothers. However, for fathers, the percentage not known for risk factors pertaining to unrealistic expectations of the child, harsh punishment of the child, and child perceived as being difficult were higher (see Figure 4.5).

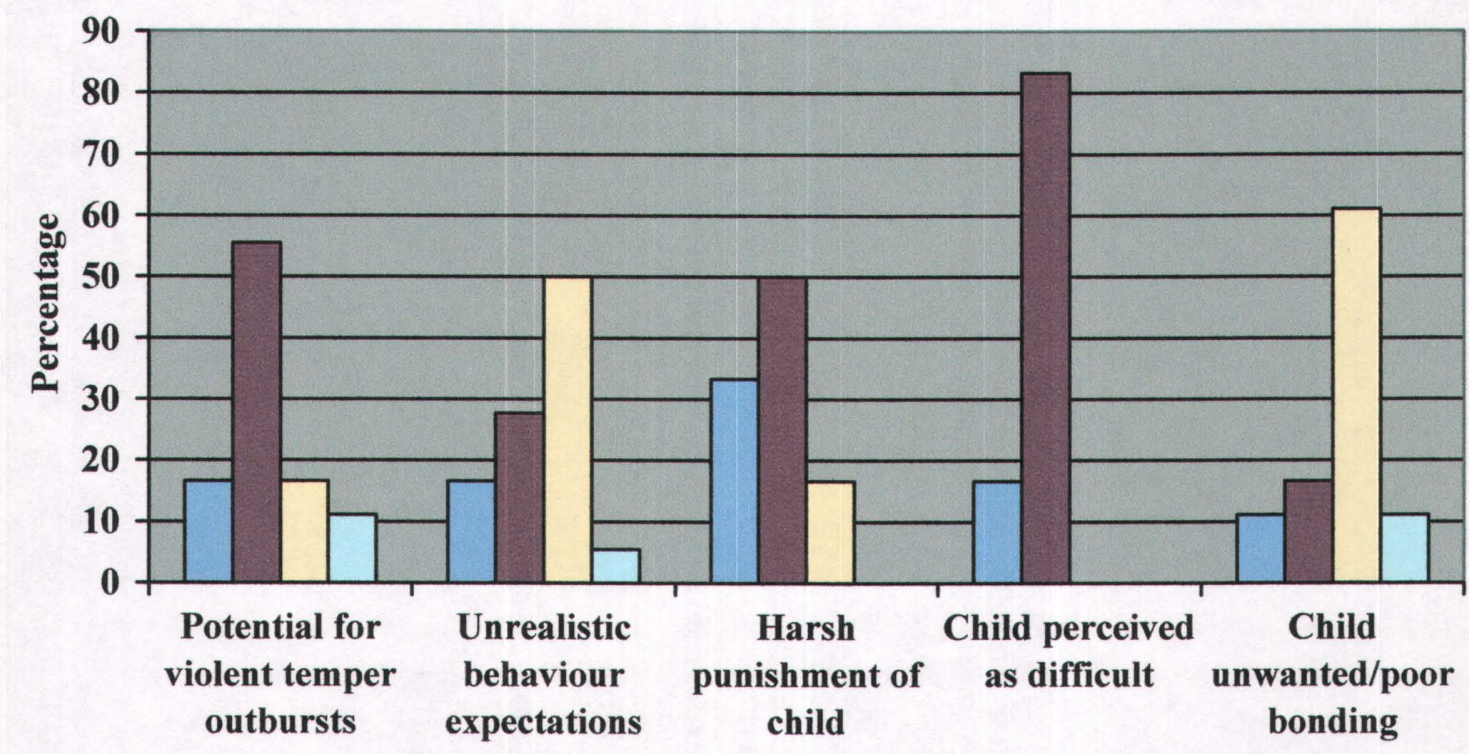

$\square$ Unknown $\square$ No Problem $\square$ Mild Problem $\square$ Severe Problem

Figure 4.3. Birth Mother Family Stress Checklist Score: Items 6-10 


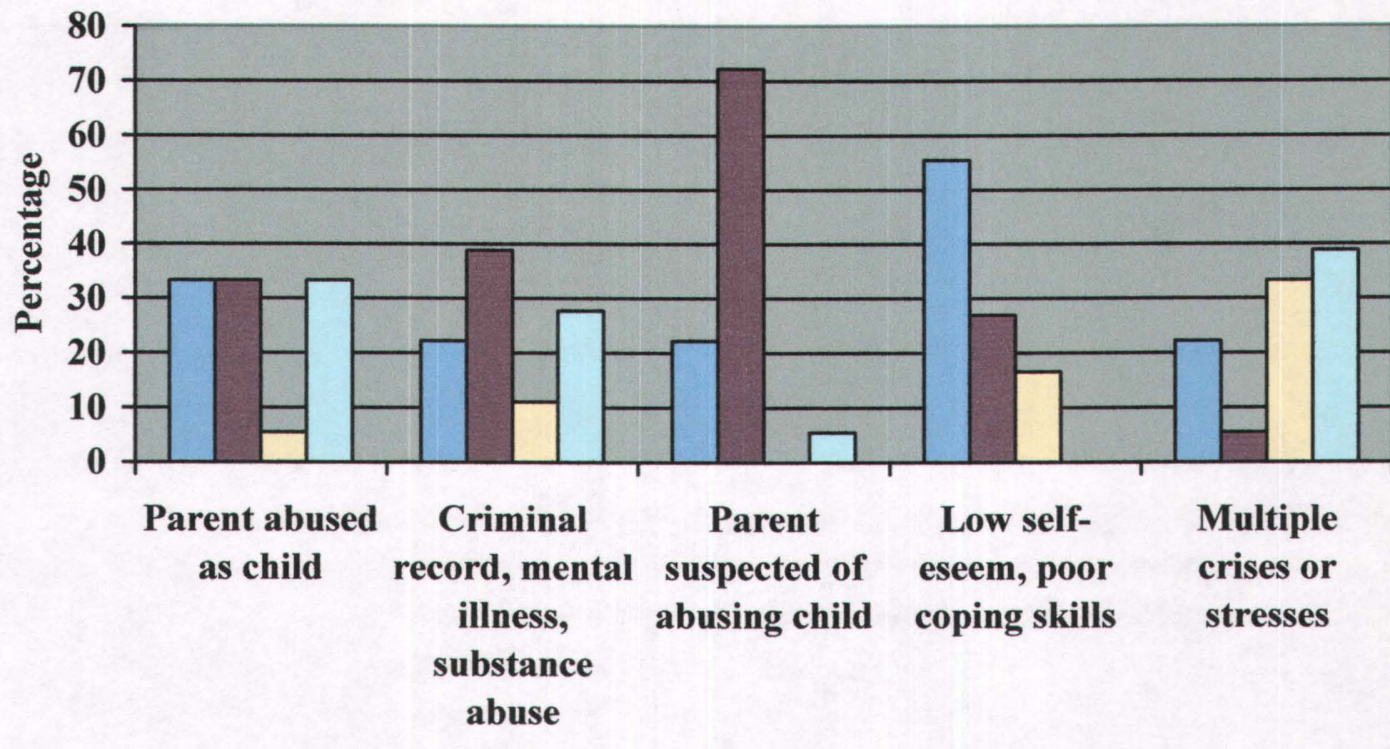

\section{Unknown $\square$ No Problem $\square$ Mild Problem $\square$ Severe Problem}

Figure 4.4. Birth Father Family Stress Checklist Score: Items 1-5

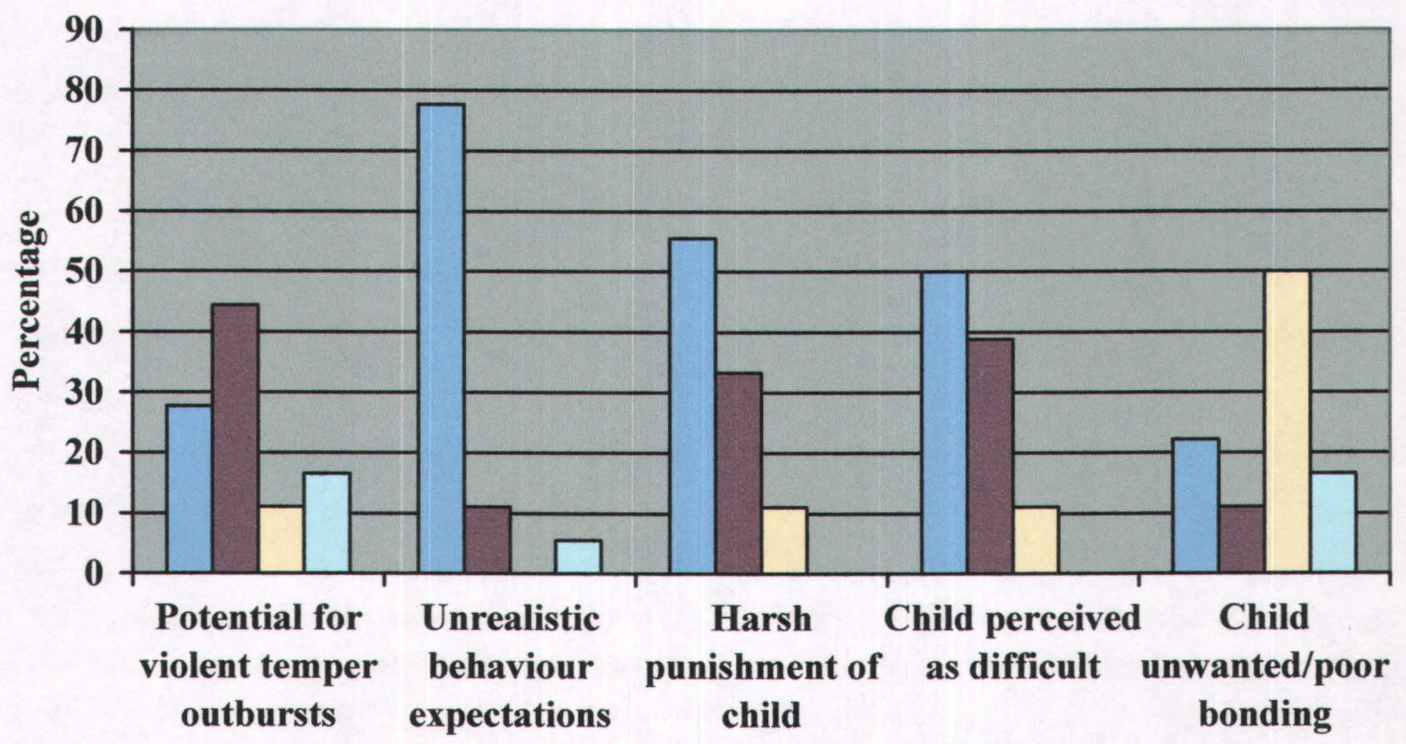

\section{Unknown $\square$ No Problem $\square$ Mild Problem $\square$ Severe Problem}

Figure 4.5. Birth Father Family Stress Checklist Score: Items 6-10 
What these preliminary results indicate is that the parents receiving home visitations have elevated levels of psychosocial risk in the areas of substance abuse, criminal behavior, and mental illness. However, elevated risk factors as they relate to abuse and neglect of the child or harsh parenting practices are not reported.

The home-visitation records of Parenting Plus were abstracted to include the following indicators of service delivery:

1. The number of families in the program;

2. The length parents have been participating in the program;

3. The frequency of home visitations conducted by the paraprofessionals; and

4. The current program level of participating families.

The decision to abstract the rates of service delivery as indicated was made to provide a baseline from which the program could be monitored. As well, this information lends an understanding of how the program has been operationalized. When the number of parents enrolled in the program is compared to their length of time in program, it is found that a greater number have joined recently (see Figure 4.6). When the total number of parents that either remain or have been discharged from the program are compared, it is found that over $58 \%$ have been in the program for one to three months. According to Parenting Plus family support plans, of these same families, $16.7 \%$ entered the program at the prenatal level, $66.7 \%$ entered the program at Level 1 , and $16.7 \%$ entered at Level X which practices creative outreach. If these same families are compared to their status in the program, it is found that $66.7 \%$ are on Level 1 and $33.3 \%$ are on Level X. What these results indicate, from the perspective of service delivery, is that FSWs, during Parenting Plus's first year of operation, spent more time engaging the families by establishing trust 
and rapport-activities characteristic of being placed on Level 1. Subsequently, no families participating in the program were on Levels 2,3 , or 4 , where goal setting with the family would occur as one of the primary activities carried out during home visitations. However, these are preliminary results and reflect a program that has been evolving and developing over a relatively short time span of 13 months.

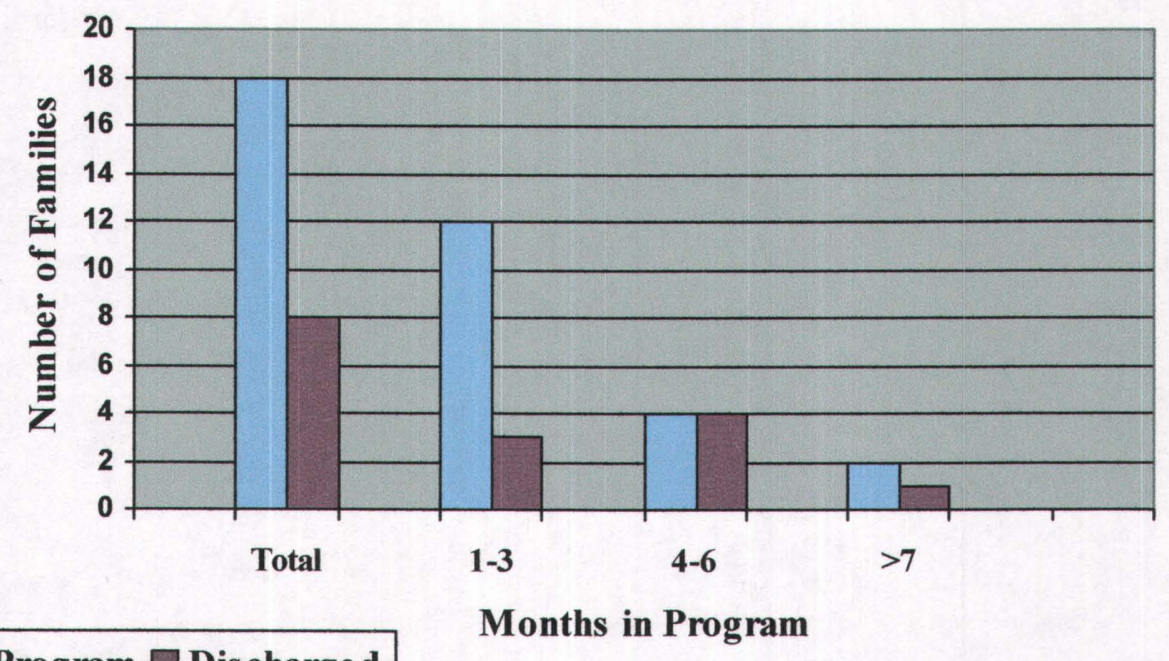

Figure 4.6. Length of Time in Program, Parenting Plus: October 2000.

When the frequency of home visits as based on length of time in the program are compared, it is found that participants receiving services for one to three months have had on average between three to four home visits each, compared with those participants discharged after one to three months, who average only one home visit (see Figure 4.7). However, for those in the program for four to six months, the number of home visits declines slightly. Why these participants might have fewer visits can be explained by mobility, a declining interest, and increased efforts directed by program staff towards enrolling new families in the program. Those participants who have been in the program 
for over seven months have a higher number of home visitations, as would be expected, with more extensive contact with the FSW over time.

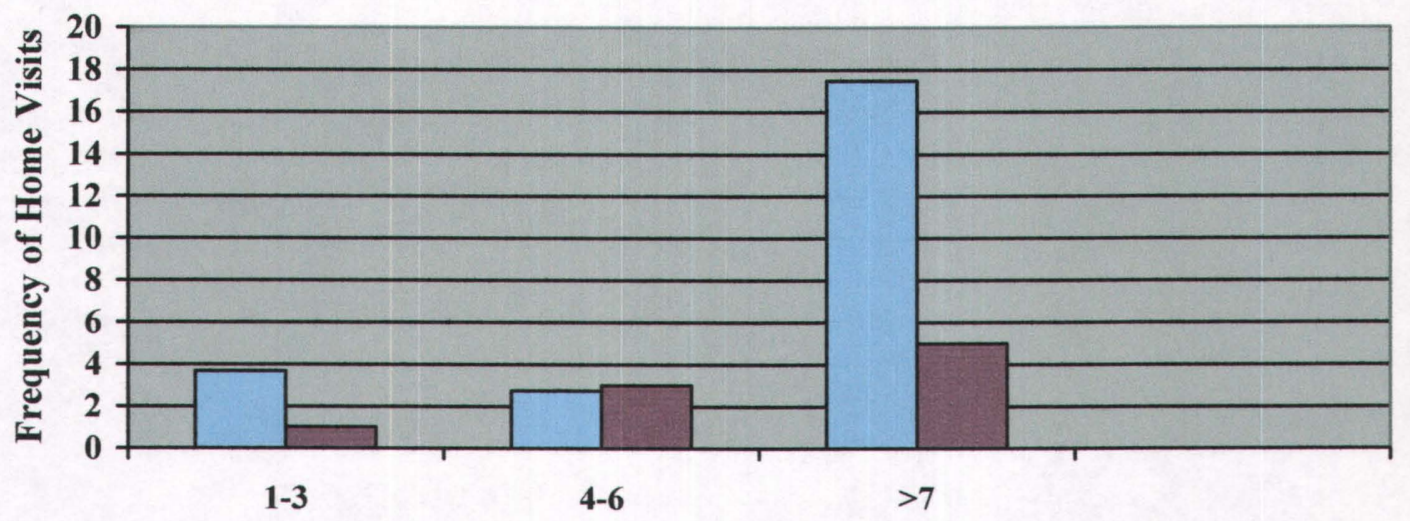

In Program $\square$ Discharged

Months in Program

Figure 4.7. Home Visit Frequency by Length of Time in Program, Parenting Plus: October 2000.

Besides the number and length of actual home visitations conducted by the FSWs, other types of service delivery pertinent to the discussion include attempts to contact participants either in person or over the telephone. These types of service delivery are relevant to the implementation evaluation because they are indicators of program delivery. Furthermore, they underscore the amount of time and effort expended by the FSWs in an attempt to engage participants in home visitations that do not always result in direct contact. The rates of service delivery abstracted for those either in the program or discharged indicate the following averages:

1. 4.5 home visits have occurred;

2. 7.5 hours of home visitations per family have been received;

3. 2.2 hours is the average length of a home visit; 
4. 1.18 home visits have been attempted per family;

5. 3.5 telephone contacts have been attempted per family; and

6. 3.8 telephone contacts have been made per family.

The results point out that, generally, families participating in the program have received 5 visits from their FSW lasting an average 2.2 hours in length each. However, to use these rates alone as indicators of service delivery would be misleading. For example, attempted home visits by FSWs ranged from 1 to 6 , with an average of 1.18 attempted home visits per family. Therefore, it would appear that some families have proved more difficult to engage in home visitations than others have.

\section{Interview Results}

The stakeholders interviewed for the evaluation included: health care professionals involved in screening from Regina General Hospital; public-health nurses and community-health nurses from the Pipestone Health District; Parenting Plus program staff; a local physician; the former CEO of the Pipestone Health District; the Early Childhood Intervention Program co-ordinator in the Pipestone Health District; and a representative from the corporate sponsor SaskTel. In total, over 23 individuals were interviewed using semi-structured depth interview methods.

The inductive analysis results for the semi-structured depth interviews reveal 4 major dimensions that were derived from 50 raw-data themes. The 4 dimensions abstracted from 19 first-order categories and 8 second-order categories include: Partnership Building and Communication, Screening and Assessment Concerns, Challenges to Program Model, and Lessons Learned. Although the dimensions are represented as 
distinct, they are interrelated and interwoven across raw-data themes, first-order categories, and second-order categories. In reporting the results, representative quotations from the transcripts are interspersed to clarify the identified major dimensions.

\section{Partnership building and communication}

The Partnership Building and Communication dimension is mentioned by 23 of the stakeholders interviewed and emerges from 5 first-order categories. The 10 raw-data themes emerge into 2 second-order categories: Ineffective Communication and Challenges to Partnership Building. Ineffective Communication is derived from 6 rawdata themes that relate to Lack of Feedback, Poor Role Clarity, and Poor Preimplementation Communication. Challenges to Partnership Building is derived from 4 raw-data themes that relate to building community capacity and peoples' experiences (see Figure 4.8).

The second-order category, Ineffective Communication, reflects three independent but interrelated first-order categories-Lack of Feedback, Poor Role Clarity, and Poor Preimplementation Communication. The first-order category, Lack of Feedback, is derived from two raw-data themes. The first relates to the lack of communication conveyed about the home visitations, while the second refers to lack of feedback on the program's development, service delivery, and outcomes. Health-care practitioners who provided care to the mothers and infants, either through community or district health centers, viewed feedback as desirable and integral to maintaining the health and well-being of families. The following depicts how feedback surrounding the home visitations would aid in communication and partnership building: 
What I would like to have better, is. . .more feedback from the worker that is providing service here. I have not actually heard from her. And I'm not really aware of what her areas of concern are with this client, what they're working on, and I'd kind of appreciate some feedback from her. (Tr. 15, p. 2)

$\begin{array}{llll}\text { Raw-Data Themes } & \begin{array}{l}\text { First-Order } \\ \text { Category }\end{array} & \begin{array}{l}\text { Second-Order } \\ \text { Category }\end{array} & \text { Dimension }\end{array}$

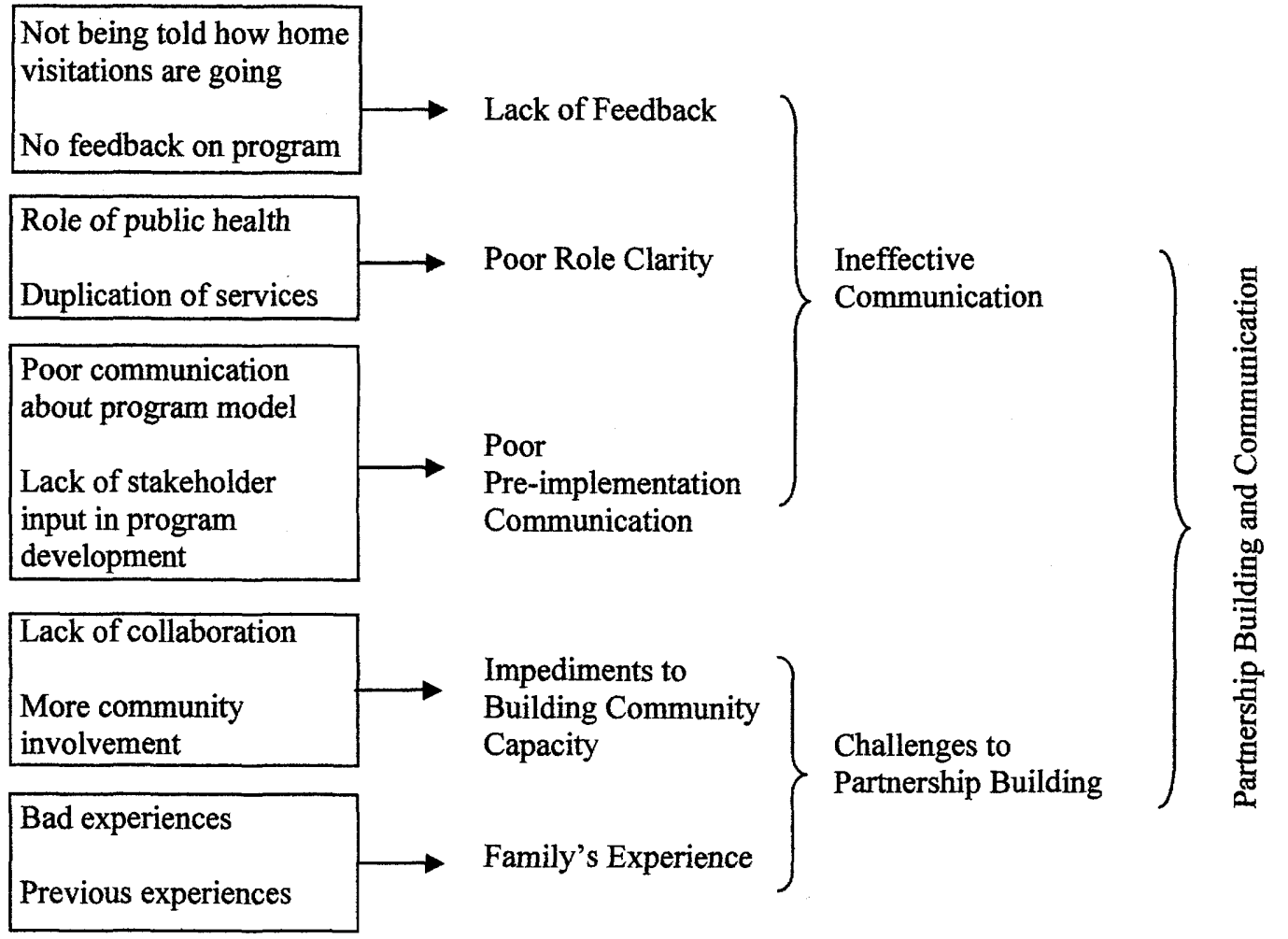

Figure 4.8. Partnership Building and Communication Dimension

Stakeholders also spoke about the need for feedback in more general terms. Feedback was viewed as desirable because it would aid in the promotion and development of Parenting Plus in the health district by building relationships. Thus, the need for responsiveness as it relates to the program's development and service delivery is appropriately expressed by one of the stakeholders in the following quotation: 
. . providing feedback to the communities and stakeholders. And you just need to continue promoting the program with maybe people, friends that I have or anything. Some of them don't know that it's out there. Having the information available at prenatal classes or parenting classes or something so that people know that the program is out there and that they can utilize it. Making everybody feel like they are in the program. Continuing to collaborate and get feedback to all sectors like First Nations, health districts, and social services, that kind of thing. (Tr. 12, p. 5)

Here feedback is viewed as integral to fostering effective practices for developing intersectoral programs. The second-order category, Ineffective Communication, is also derived from the first-order category, Poor Role Clarity, which emerges from the rawdata themes relating to the role of health-care practitioners and the perceived duplication of early intervention services in the Pipestone Health District. As Parenting Plus is a new pilot program in the health district, geared towards the identification of overburdened families for the provision of paraprofessional home visitations, the role of public health is integral to the delivery and operationalization of the program. Unfortunately, due to poor communication practiced during the implementation phase of the program, there was a perceived ambiguity as to the role public health would play in the operationalization of Parenting Plus:

We had listened to her spiel to all the nurses. And it was kind of vague at the beginning, how it was to be delivered and what we were to do, as giving this referral right to this lady and that sort of thing. And it came to our office because we pass on referrals. So that was where I think there was some confusing information.

(Tr. 11, p. 2)

Stakeholders also felt that the lack of role clarity might result in duplication of early intervention services in the district, as the following quotation illustrates:

We knew what was being requested. You'll probably hear repeatedly from some of us that we felt that a lot of this was already being done, and I think the program possibly could have been managed through public health just because we are very aware of a lot of these families already and the background. . . It would have helped if there was more communication and co-ordination between the two programs. (Tr. 23, p. 3) 
Unfortunately, as with many pilot projects, the time lines in place for the planning and development of Parenting Plus were not adequate. This put Parenting Plus in a position of having to be implemented in order to meet funding criteria, without having the opportunity to do the work necessary for program development and partnership building. In turn, some of the difficulties encountered during the implementation phase of the program were exacerbated by poor pre-implementation communication. As a first-order category of the second-order category, Ineffective Communication, Poor Preimplementation Communication arose from the raw-data themes relating to poor communication about the program model and lack of stakeholder input in program development and implementation:

I think this program started without consulting or having people part of it, and they just felt that things happened and they do not know about it, they were not involved in decision-making. I know it happened really fast, because they got the grant and submitted the proposal and there was a deadline. A lot of relationship problems started because of that. (Tr. 20, p. 5)

The previous quotation illustrates how ineffective communication practices at the developmental stage can effect and are closely related to partnership building. This demonstrates how interwoven the sub themes are and their complex interplay in the major dimension, Partnership Building and Communication.

The other second-order category, Challenges to Partnership Building, emerges from the two first-order categories Impediments to Building Community Capacity and Family's Experience. Specifically, the first-order category, Impediments to Building Community Capacity, is derived from raw-data themes relating to lack of collaboration and community involvement. The lack of collaboration that stakeholders experienced during the implementation process and the detrimental effects it had on establishing 
partnerships and building community capacity is illustrated by the following:

Of course they are going to make their own decisions, seeing it's their program, but if they have other people who have experience in the field, who can offer ideas, I thought it was a good way to partner, and again, they didn't feel that this was really necessary. So it was really unfortunate. I spent a lot of time chasing and offering and really trying to partner with them, and they just wanted to go off on their own.

(Tr. 2, p. 3)

Also:

Well initially when the program first started it was me doing all the contacts. I was trying to get involved, become involved. I firmly believe in partnership to avoid duplication and to provide families with an overall program without segmented services coming in. So I really made great efforts to go out and be a partner and was pretty much dismissed. (Tr. 2, p. 3)

The first-order category, Family's Experience, is derived from raw-data themes relating to previous experiences with other agencies and potentially bad experiences in the program. The following comments made by a stakeholder illustrate potential difficulties in establishing trust and engaging families in the program due to previous experiences:

For the Parenting Plus program I introduce it to them and I tell them what it is all about and these people are very sensitive because they figure it's got something to do with child or family services. Be very careful; make sure they understand what the program is. Because I know from the start, we had a few problems when it started because people just said, no I'm not interested, because they thought it was child or family services and the children might be apprehended or something like that. And it is that fear, all the time, is it an intervention of another program like child and family services? They don't know the people, they don't know what it's all about.

(Tr. 13, p. 2)

Further:

So it takes a while for any new program to get going on reserves. And people asked is it an intervention, like another program like child and family services? (Tr. 13, p. 3)

Clearly, the challenge to effective partnership building for intersectoral programs is to overcome past experiences with other services that taint a potential participant's current perceptions of the program. This is even more significant for rural health districts when 
already difficult intersectoral relationships between federal and provincial governments and First Nation communities are exacerbated by historical prejudices.

\section{Screening and assessment concerns}

Parenting Plus follows a two-stage screening and assessment process for the identification of families at-risk that is modeled after the Hawaii Healthy Start Program. The model calls for a medical record screen to be completed by a health-care practitioner for all live births from the health district. The screen is then forwarded to Parenting Plus where it is scored. If the screen is positive or seven or more items are unknown, the family is offered an assessment by the program co-ordinator. Based on a positive assessment with the Family Stress Checklist (Kempe, 1976), the family is offered homevisitations by Parenting Plus. If the family does not refuse the program but is not interested in home visitations at the present time, another service delivery option exists. At the discretion of the family, Parenting Plus paraprofessionals continue to maintain contact for a four-month period. After four months, if the family has not accepted services, they are dropped from the caseload. This type of service provided by the paraprofessionals is described as creative outreach and is an entry point for families into the program.

In conducting an inductive analysis, the second major dimension derived from the semi-structured depth interviews with stakeholders is Screening and Assessment Concerns (see Figure 4.9). The issues identified here are mentioned by 21 of the stakeholders interviewed and emanate from 5 first-order categories. Ten raw-data themes merge into 2 second-order categories, Screening Concerns and Assessment Concerns. 
Screening Concerns is derived from 6 raw-data themes that relate to Relevancy of the Screen, Challenges to Completion, and Ethical Considerations. Assessment concerns is derived from 4 raw-data themes that relate to Offering Services and Families Assessed.

$\begin{array}{llll}\text { Raw-Data Themes } & \begin{array}{l}\text { First-Order } \\ \text { Category }\end{array} & \begin{array}{l}\text { Second-Order } \\ \text { Category }\end{array} & \text { Dimension }\end{array}$
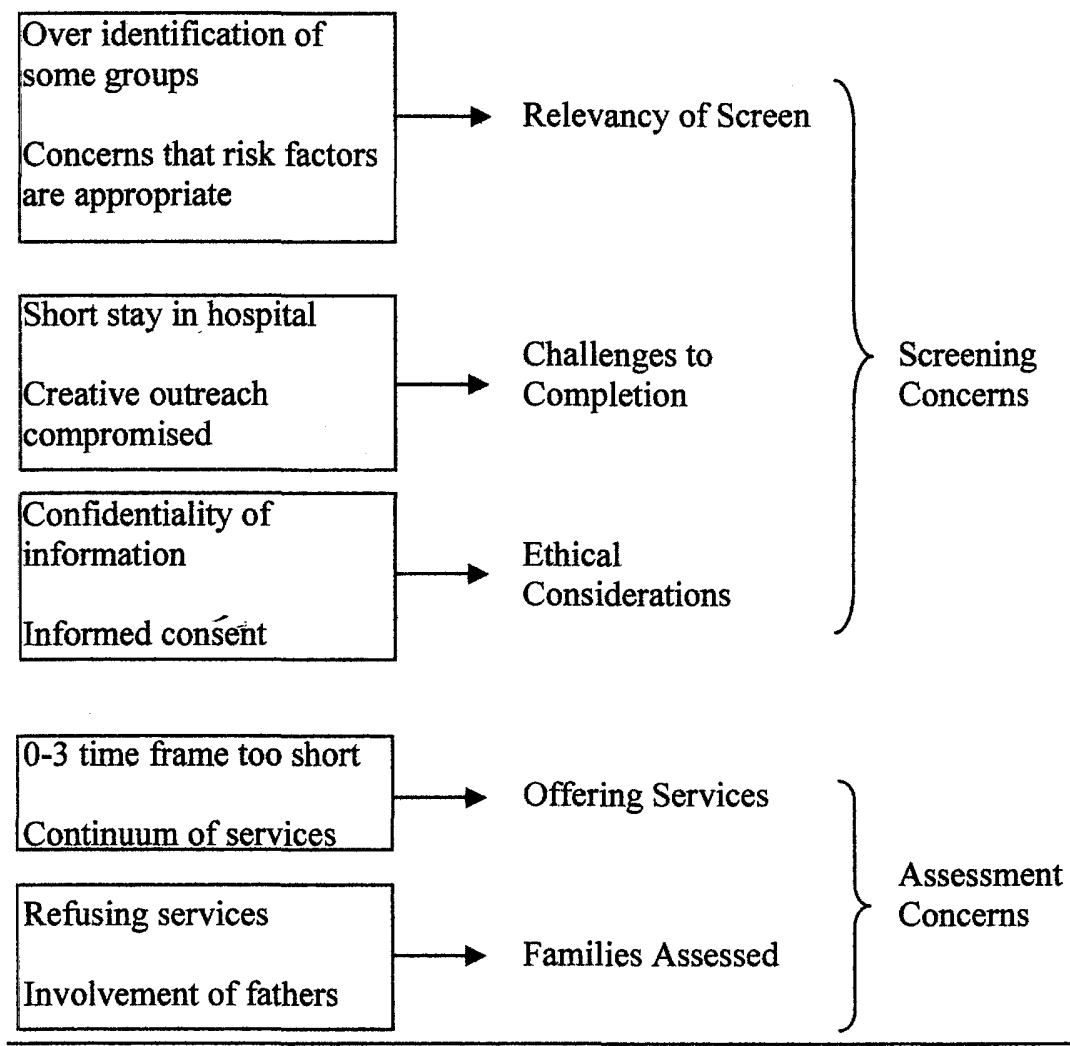

Figure 4.9. Screening and Assessment Concerns Dimension

The first-order category, Relevancy of the Screen, is composed of two independent but interrelated raw-data themes that include over-identification of some groups and inappropriateness of risk factors on the screen. Screens were to be completed by out-ofdistrict hospitals, district nurses, and physicians. In implementing the model, a number of concerns were raised regarding the relevancy of the screen. Stakeholders asked questions 
pertaining to the accurate identification of families at-risk and whether the risk factors, because of their inclusion on the screen, were good predictors of psychosocial adjustment:

So had we had a little bit more information maybe even some background on the literature search on why they need to know the criminal history. Why do they need to know? What has shown? Why is this a risk factor? The unsuccessful abortion. Why is that a risk factor? (Tr. 8, p. 3)

The other raw-data theme for the first-order category, Relevancy of Screen, is the potential of the screen to over-or under-identify families at psychosocial risk. The following quotation from a stakeholder is representative of this concern:

Sometimes the form looks like it's going to be heavy duty high-risk, a lot of details are involved. And we find they are really together and they're doing okay. But, sometimes it's the other way around. The form is blank and it looks like they're fine, and you go there and they are quite the opposite. (Tr. 7, p. 9)

The second-order category, Screening Concerns, is also composed of the first-order category, Challenges to Completion, that emerges from the raw-data relating to creative outreach being compromised and difficulties in completing the screen. Hospitals that are outside of the Pipestone Health District where the vast majority of live births occur, agreed that screens for all live births for the health district would be completed by hospital staff and forwarded to Parenting Plus. However, the birth mother's brief stay in the hospital, often only 48 hours, makes the collection of information pertaining to the completion of the medical record screen for Parenting Plus difficult to obtain. The difficulties encountered in completing the screens due the birth mothers short stay in the hospital is appropriately expressed by one of the stakeholders in the following representative quotation:

You know some of the information I was always a little bit concerned about anyway. One that has come up is the criminal record of the family. I don't think it is 
information we really access. Some of the families we have for a very short period of time. (Tr. 6, p. 5)

Other screening concerns that proved to be a challenge to the completion of the medical record screen are reported as a raw-data theme regarding the compromise of creative outreach. This was most apparent for those screens involving First Nation families. Although the screens were being completed and forwarded to Parenting Plus, only the names of those families who screened positive and were interested in the program were received. Thus, the ability for Parenting Plus to practice creative outreach with those First Nation families who screened positive and potentially might have a positive assessment was thwarted because program staff were not allowed contact with these families. The potential difficulties in practicing creative outreach for First Nation families is expressed in the following stakeholder quotation:

The federal health nurses on the reserve are quite willing to use our screens and screen all newborns. But, if the family refuses, they don't send us the positive screen. They have agreed to send us the number, so we have a statistical base like we had 25 positive screens and 20 agreed to be on the program. We can still get those numbers but we don't actually know what families that were positive screens and had refused any further involvement. And I guess the difference that happens there is, if we have a positive screen and we do an assessment of the family, they're quite within their rights to refuse the program, and they do. But, then we do what the family support workers call creative outreach. They phone a family, they give them a little gift or whatever and they talk to them further about the program after a month or two. And you know, maybe they make contact a couple of times and just see, as things progress, maybe the family has changed their mind. So they sort of leave the door open, so that if a family changes their mind they can still get on board, where on a reserve if the family has a positive screen but they decide they don't want to be on the program, we don't even know their names, so we can't contact them and leave the door open. We don't have that opportunity. (Tr. 18, p. 7)

The last first-order category that results in the emergence of the second-order category, Screening Concerns, surrounds ethical considerations. Ethical considerations that stakeholders experienced pertinent to the medical record screen for Parenting Plus 
relate to confidentiality of information obtained and informed consent. Concerns expressed by stakeholders regarding confidentiality of information surround some of the items on the medical record screen completed by health-care practitioners in the district and the subsequent forwarding of confidential information on the families obtained to Parenting Plus. The following stakeholders refer to these concerns:

Certainly issues of confidentiality were raised by us and we kind of dug our heels in and I know that it caused a bit of tension. We felt we had to clarify with our governing body as to what would happen to us if we were forwarding very personal information to people without all of these people, clients, being totally aware of that, and then we were afraid of the repercussions. You know, where did you get that information? Well, how did you get it? Who gave the right to release that information on me? Some of that information is lots of health history in there that really is nobody else's business unless they want it to be. And as well, financial status and things like that of the family. (Tr. 23, p. 1)

In addition:

The next part we heard, was when we were being invited to be involved, it was once the program was already off the ground and they had at first wanted public health to do the screening and which was also a time commitment on our part. ... We weren't sure besides the fact that we were not going to be involved otherwise in implementing the program and we weren't very comfortable with doing the screenings and handling all the information over to somebody else. (Tr. 23, p. 3)

Along with confidentiality of information, informed consent is an issue for stakeholders concerning obtaining information for the completion of the screen. They questioned whether informed consent was being obtained from parents in the completion of the screen. The following stakeholder depicts how the raw-data theme pertaining to informed consent is viewed as an ethical concern relating to the screening of birth mothers for Parenting Plus:

...you know the screening is a very big concern of mine. I think great care has to be taken in the screening and the diplomacy in the consent factor. I know that when you are in the hospital that you sign an overall paper, or overall thing, I agreed to be provided with services. But I think we have to be careful for the intrusive way that this is done. I asked the question at the meeting about consent. Does everybody have 
consent? Or does everybody consent to have the screening done. And not everybody does apparently. I mean the idea is right but families still need to be informed. And as far as the ethics, like who is checking into the ethics of this kind of material?

(Tr. 2, p. 7)

The final second-order category for the major dimension Screening and Assessment Concerns is Assessment Concerns. As a second-order category, Assessment Concerns emerges from the first-order categories, Offering Services and Families Assessed. As a first-order category, Offering Services is derived from raw-data themes pertaining to the time frame for admittance into the program and the provision of a continuum of services. Parenting Plus, as modeled after Hawaii Healthy Start, only accepts families in the program who have newborns up to three months of age. However, stakeholders believed that the program could more effectively reach its target population if the age of acceptance were expanded. It is the belief among stakeholders that the birth to three month period is not necessarily a time of perceived stress by the family in which the provision of strength-based home visitations is deemed warranted. This opinion is expressed by the following stakeholder:

Because the mother has no problems zero to three, she is not seeing parenting as an issue. She has not even got over the delivery except she has a baby in the house. So that is why they could not be having referrals or not accepting services. (Tr. 14, p. 5)

And,

One of the challenges is definitely they are not ready for it [Parenting Plus] at three months. They may not even be ready for it at one year. But as they come along to issues. . You have a problem, you have a crisis or issue then say. . I need some help here..(Tr. 14, p. 5)

The other raw-data theme contributing to the first-order category, Offering Services, relates the items on the screen accurately identify families who are at-risk and how families just below the cut-offs used might also benefit from home visitations. Thus, the 
need to establish a continuum of services for families is viewed as beneficial as it would

ultimately result in more families being able to access home visitations:

...there are some parenting risk factors, but they're lower than the assessment would give them, so they wouldn't qualify for a home-visiting program. And I think that is great. But often in situations of child protection, my experience has said that child protection services can put in other programing to supplement what goes on to the families. So I think we might be missing a group of people who can really benefit, that are at-risk, not as high risk that the indicators say, but could benefit from the home based program.(Tr. 6, p. 4)

In addition:

Even extended into not only early childhood programs for the high risk but the middle risk families that are just lacking support, they have the other areas of their life intact, but they still don't have all the extended support that they need, so they survive, but they just survive. (Tr. 8, p. 9)

The second-order category, Assessment Concerns, also evolves out of the first-order category, Families Assessed. Families Assessed relates to raw-data themes that include families refusing services and the involvement of fathers. Stakeholders expressed concerns regarding how to engage and follow up with those families that refuse services but whose assessment is positive. The following quotations depict the two raw-data themes for Assessment Concerns.

I've got one mom that I did the assessment with and it's positive. I found a few times she said, she will discuss it with her husband, and that could be part of the problem, but you get the feeling it's just to postpone. So I've phoned a few times, and she says oh she hasn't discussed it with him yet, that's all excuses like that. So I thought maybe I would stop by and drop some information off and just find out how she is doing and how the baby is doing. Until she tells me I'm not interested in this program or don't come again, or don't send any information and then I mean you can't really force yourself on them. What I did in cases like that, if it is positive and they don't want to be part of the program, I usually phone the health nurse, and tell her I did the assessment, it was positive, and they don't want to be part of the program.

(Tr. 21, p. 5)

and, 
For some reason there is not always that many men involved. It is hard to get the fathers, some don't have fathers. They are never around, so you cannot assess them. It is always the mother. (Tr. 25, p. 9)

The original model for Parenting Plus called for screening and referral to be completed by out-of-district hospitals and by district nurses and physicians. In implementing the model of service delivery, it was found that the majority of live births occur out of the health district. At the time of the evaluation, agreements had been reached with Regina General hospital, the Brandon hospital, Ochapowace, Kahkewistahew, Carry the Kettle, and Saskimay, with agreements pending for Cowesses and the Yorkton hospital. As well, public-health nurses in the Pipestone Health District are now providing screening referrals for out-of-district hospitals in Melville and Yorkton and to birth mothers living in communities that might otherwise have been missed. Thus, with the anticipated signing of the final two agreements and public health's participation, all live births for the Pipestone Health District are effectively screened. Although the model of service delivery called for medical record screens to be completed by the referral source, this proved unworkable for Parenting Plus. The birth mother's brief stay in the hospital made the obtaining of information to complete the screen difficult to procure. Consequently, Regina General now faxes all medical referrals of live births in the Pipestone Health District to Parenting Plus, with the district public-health nurses providing all screens and pre-natal referrals that they receive as well.

If the screen is incomplete, the Parenting Plus co-ordinator contacts the birth mother on the telephone or in person if necessary. If this results in a positive screen or if there are seven or more unknowns, the program co-ordinator conducts a family assessment during one or two home visits. The Family Stress Checklist (Kempe, 1976) is used to determine 
if the family is overburdened and what degree of psychosocial stress it experiences. Consequently, the results of this more intensive effort to provide a systematic screening to families of newborns in the Pipestone Health District has lead to a significantly higher number of screens being received over the last five months.

For those families of newborns residing in First Nation communities, the federal community-health nurse completes the medical screen and refers to Parenting Plus only those families that are positive and are interested in receiving Parenting Plus homevisiting services. Unfortunately, this has compromised the compilation of program statistics, as only those receiving positive screens and interested in the program are referred to the program co-ordinator for assessment. Subsequently, the number of negative or incomplete screens is not known. Second, if a family is assessed as positive but is refusing the program, they cannot be placed on Level X, where the FSW is able to practice creative outreach. Thus, the model of service delivery is significantly different $\ldots$ for First Nation communities, as follow-up to screening and assessment in the form of creative outreach is not always possible to conduct.

\section{Challenges to Parenting Plus}

The third major dimension that emerged from the semi-structured depth interviews for Parenting Plus and that is mentioned by 20 stakeholders is Challenges to Parenting Plus. Challenges to Parenting Plus embodies challenges to the service delivery and sustainability of the program. It is derived from 2 second-order categories that emerge from 5 first-order categories and 10 raw-data themes (see Figure 4.10).

The second-order category, challenges to service delivery, emanates from three first- 
order categories: Being a Rural Health District, Negative Perceptions of the Program Model, and paraprofessional knowledgeableness. The first-order category, Being a Rural Health District, emerges from three raw-data themes pertaining to the geographical mobility of participants, associated travel costs in a rural health district, and the amount of time involved in trying to contact and engage families.

$\begin{array}{lll}\text { Raw-Data Themes } & \begin{array}{l}\text { First-Order } \\ \text { Category }\end{array} & \begin{array}{l}\text { Second-Order } \\ \text { Category }\end{array}\end{array}$

Geographical mobility of participants

Travel costs

Being a Rural

Health District

Time factor

Program model based on
Hawaiian experiences
Program model more
appropriate for urban
setting

Education of
paraprofessionals
Accessibility of training

\section{(n)}

Negative perception of early intervention Need for outcomes

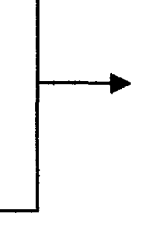

(1)

Figure 4.10 Challenges to Parenting Plus Dimension

The geographical diversity of a rural health district creates significant challenges to service delivery for Parenting Plus. Stakeholders involved in program delivery spoke about the mobility of participants, which made it difficult for home visitations to occur on 
a consistent basis with some families:

I think I could tell you from their point of view that one of the biggest challenges they're facing is the mobility of clients. They go expecting to meet with the family and they're gone. Like not just gone for the morning, they've moved to Regina. From the week before till now, that's probably one of the frustrations they're facing or one of the challenges. (Tr. 18, p. 15)

The second raw-data theme for the first-order category, Being a Rural Health District, regards the additional costs associated with traveling in the Pipestone Health District for the provision of home visitations. The following typifies the concerns expressed regarding the additional costs to the program incurred due to the districts' expansiveness as an impediment to service delivery:

I mean it adds a huge cost to the program that makes it less cost efficient. And I mean I wish it wasn't a consideration, but it just is, and you have to be cost efficient, and it seems to be one of the areas that we're really struggling with, is the amount of time staff spend on the road and the travel dollars that are attached to that. (Tr. 19, p. 5)

The third raw-data theme that emerged as a challenge to the delivery of Parenting Plus in the Pipestone Health District relates to the amount of time that travel would consume in the day-to-day duties of program staff. As a result, stakeholders spoke about how the size of the Pipestone Health District effected home visitations and the amount of contact that the paraprofessionals were able to make and maintain with participants:

For this district, I think the distance is a problem. We did not really think that distance would have an effect on a program like this. To travel to McLean is an hour and $a$ half and an hour and $a$ half back to the office here and then you have a two or a two and a half hour visit with the families, so it's one visit a morning. And then, I know we try to do the reports as soon as possible after that so that we don't lose any information. So the afternoon you spend doing your report. So it's time consuming. (Tr. 21, p. 7)

Further:

Well travel is certainly one of the aspects that take up a lot of time. One of the issues is that a Family Support Worker may spend two hours at home-visiting a family, and four hours on the road to get there and get back. So she is actually spending, you 
know like I guess I'm wondering if there is a better way, can we do this so that there is not as much travel and but then I mean it would involve people who aren't full time, so then you run into problems of less education and less commitment.

(Tr. 19, p. 1)

The second-order category, Challenges to Service Delivery, was also derived from the first-order category, Negative Perceptions of Program Model. This first-order category emerges from two raw-data themes related to the Hawaiian roots of the program model and the appropriateness of the program model in a rural setting. Stakeholders questioned the applicability of the Hawaii Healthy Start model because of their perceptions surrounding the implementation of a program based on Hawaiian culture. Although, Hawaii Healthy Start has been implemented in Canada, stakeholders seemed to be unaware of this and, as a result, tended to view the program model in a negative light:

...the Hawaii program is based on the Hawaiian culture. And does that all transfer over to being utilized in our culture? The native culture? There are a lot of differences. Thank goodness we do have one of the workers in the Parenting Plus program who has a native background, and hopefully, we can really work with those families. (Tr. 23, p. 5)

Also:

. . .I think they should be marketing their program more like ECIP instead of following a set thing from Hawaii which is for Pete's sake, from the States. You see, there is another whole issue. It was just a program parachuted in and it wasn't to our context here in Saskatchewan. We're rural. We're different than Hawaii.

(Tr. 14, p. 5)

The other raw-data theme from which the first-order category Negative Perceptions of the program model is derived is connected to the applicability of the program model in a rural health setting. The perception is that Hawaii Healthy Start is an urban program and that associated costs and travel time would be less of an impediment if the program was implemented and delivered in an urban, as opposed to rural, health district: 
I think. . it would probably work much better if it's in a city-all families in one area and all services in one area, I think Hawaii Healthy Start was also in a big city. It's just more positive, everything's more positive. Access for families to and from services and support groups and the traveling for families, everything is just easier in the city. (Tr. 21, p. 13)

The final first-order category that resulted in the emergence of the second-order category, Challenges to Service Delivery, is Paraprofessional Knowledgeableness. The raw-data themes from which Paraprofessional Knowledgeableness is derived include issues pertaining to the education and accessibility of training for the paraprofessionals.

Stakeholders questioned whether it was the best practice to provide a service delivery to high-risk families that is delivered by paraprofessionals:

. . the paraprofessionals that they've hired, nice people, but there again, they came on without their education. We've asked at times for their level of education, or what their qualifications were, and you've probably heard this from others, and we have never been told. And they didn't get their training right away, they have been going out and doing home visits already. (Tr. 10, p. 3)

Also:

I think what they have to look at in this program is the quality of their workers, the quality of education, because it will be found out. If they come to the homes and they are not prepared in the family dynamics and all the things that you need to have, I don't believe that the level is there yet. I mean if it takes four years for public nurses to come to the home and then even a social worker, why do we think we're putting our highest risk people in the hands of people with less education. That is not fair to those people because they may be lower income, more disadvantaged, have higher determinants of health need. We shouldn't put our lower quality worker in just because we need to. (Tr. 14, p. 5)

Further:

The other thing that would be a bit of a challenge is education for your workers that are going in there; they have to be really highly educated in personal skills so that, when they go in and talk to the family, the family does not feel like they are not doing anything right. So that would be the other thing. Like, as an educator I was really interested in the education that those workers got, and how they are educated in their interpersonal skills and to keep up their relationships with those families, because that is very important. (Tr. 8, p. 9) 
The other raw-data theme that resulted in the emergence of the first-order category, Paraprofessional Knowledgeableness, is also tied into education but from the perspective of accessibility. Stakeholders spoke about the need for training to be delivered to Parenting Plus staff but reiterated how accessibility to such training was difficult to obtain. Although initial training prior to program start-up was provided, continued professional development was difficult to obtain. If training or education is not accessible, then this could be viewed as hindering the development of an adequate knowledge base for the provision of home-visiting services:

I think we all wanted to have by this time more training in the program. That would be a plus if we can, but the training is not available. So that is really a problem. And we don't have the funds to get somebody from the States to give us some training. We can't go there because it is too expensive. So there we have difficulty with that. (Tr. 21, p. 7)

The other second-order category that gives rise to the major dimension, Challenges to Parenting Plus is Sustainability. As a second-order category, Sustainability emanates from two first-order categories, Funding and Proving Program Benefits. Funding as a first-order category includes challenges to Parenting Plus's viability as illustrated by the following:

I do not know if they are going to be able to sustain themselves, or what is going to happen. There is one more year left I think. And will it keep going? Always money is a challenge. (Tr. 11, p. 8)

Also:

I think for this program to really develop its full potential we need to have money and the people backing it, and saying here it is. I think if the finances dried up the program would probably be sacrificed, as a sort of idea at this early stage.

(Tr. 10, p. 3)

The other first-order category, Proving Program Benefits, emerges from the two raw-data themes that relate to negative perceptions of early intervention services and the need for 
outcomes. Stakeholders offer these comments:

I guess one of the things just generically, is an attitude shift towards receptivity of individuals to the idea of getting involved with an early childhood intervention program. Seeing it as a positive support for their role versus total negative. Seeing it as a positive support for their role versus totally negative, focusing on the strengths of families and programs. (Tr. 7, p. 8)

Second:

But I think the thing is, what's that saying, proof is in the pudding, whatever, I think when they actually see the results, see families are benefiting from this program then maybe they will recognize that it is an important piece of the continuum.

(Tr. 18, p. 13)

Third:

Ongoing evaluation, is needed If I was to do an evaluation after a year, I would ask was any evaluation done after six months. And I think another evaluation period may be at the second year because it is something new and it is going to have significant dollars attached to it. If we are looking at tight budgets, I think a way to assist in that is to say, okay we will do another evaluation in another year and compare one year to the next. It also builds a higher level of accountability, to my way of thinking. In terms of level of service. Sometimes things go great for the first year the evaluation is done, and it carries on, and there isn't another marker there built into the system for reassessing. (Tr. 7, p. 8 )

\section{Lessons learned}

The final major dimension that emerges from the data analysis is Lessons Learned, and it is mentioned by 15 of the stakeholders. The dimension is representative of stakeholders' experiences and reflects their opinions on the two second-order categories, Implementing Early Intervention and Home-Visiting Benefits. The second-order category, Implementing Early Intervention, emanates from three first-order categories that include Developing Participatory Structures, Adequate Resources, and Creating Awareness. The other second-order category, Home-Visiting Benefits, emerges from the first-order category, Perceived Benefits for Families (see Figure 4.11). 


$\begin{array}{llll}\text { Raw-Data Themes } & \begin{array}{l}\text { First-Order } \\ \text { Category }\end{array} & \begin{array}{l}\text { Second-Order } \\ \text { Category }\end{array} & \text { Dimension }\end{array}$

Need for advisory council

Collaborate and consult

Build community

readiness before

implementing

Program resources need to be in place

Adequate

Participatory

Structures

Category

Underestimating required
resources $\longrightarrow$ Resources

Expert advocate

More public education

Creating Awareness

Implementing

Early Intervention

Connects with resources

Is a home based service

Building a needed service

Perceived Benefits

For Families

Home Visiting

Benefits

Figure 4.11. Lessons Learned Dimension

As a first-order category, Developing Participatory Structures reflects three raw-data themes that relate to the establishment of an advisory council, partnering with local health-care providers, and involving stakeholders in program development. It is found that stakeholders view the establishment of an advisory council prior to the implementation of an early-intervention program as advantageous because it provides a forum for input and decision-making, establishes linkages, and formalizes the role of stakeholders in the hierarchy of the program. The following depicts how the 
establishment of an advisory council prior to program implementation would aid in the development of participatory structures for stakeholders:

Whereas had you had the advisory committee in place, there it is already, everybody already knows about it, now you begin doing it. You do not have to start by trying to launch the program, like beginning the delivery of the program and be telling people and getting support. (Tr. 4, p.5)

Also,

Before there was any thought of an advisory committee I suggested one to them. I recommended they have an advisory committee and asked if I could be part of that. Not for making decisions on the program but just there as a support and consultant. (Tr. 2, p. 2)

The other raw-data themes that merge the first-order category, Developing Participatory Structures, include collaborating and consulting with intersectoral stakeholders involved in early intervention at the development and service delivery phases of a program. As well, the need to build community readiness is especially relevant with First Nation - communities prior to implementing a program as suggested by the following stakeholder:

They already have the program up and launched before they even have First Nations involved. Stakeholders should have been on board before they even began, especially First Nations. (Tr. 4, p.6)

Moreover, the dynamic relationship between consultation, collaboration, and involvement needs be forged when implementing an intersectoral early-intervention program that requires stakeholder participation in its delivery. The following clarifies this raw-data theme and illustrates how interconnected it is with the major dimension Partnership Building and Communication:

It was okay as far as early childhood, but internally, the staff internally, public health, mental health, addictions, felt that they hadn't been consulted initially with those people making sure that they're on board and consulting with them to see how to best implement parts of it that they buy in to. (Tr. 22, p. 3) 
Furthermore, creating participatory structures and establishing relationships takes time, especially for an intersectoral program that has a number of potential partners and recipients of service. The following depicts how the implementation of the program needed more time at the beginning for developing participatory structures with stakeholders:

And so it [Parenting Plus] was going to be developed ad hoc, in Saskatchewan without an advisory group, without community development, without readiness from the community, without partners in place and it was rushed, without all that stuff that needed to be done ahead of time. (Tr. 5, p. 3)

Furthermore,

I guess if you are looking for advice on someone else setting up a similar kind of program, I would say there needs to be more time allowed at the beginning to do the up front work, to get public-health nurses in the district, physicians in the district, and hospitals in the district, and neighboring districts all sort of ready so that you can implement the program in a smooth way when you do implement it. (Tr. 14, p.4)

The second-order category, Implementing Early Intervention, is also based on the first-order category, Adequate Resources. As a first-order category, Adequate Resources emanates from two raw-data themes relating to having resources in place and underestimating required resources. Not having resources in place prior to implementation can effect a program's development by overwhelming staff and ultimately challenging a program's implementation:

...I don't know if it was the starting up costs but there seemed to be a real shortage of resources. There just seemed to be way too much work. They were completely overwhelmed and didn't have the resources to get going. (Tr. 11, p. 6)

As well, underestimating resources required to implement an early intervention can have detrimental effects on program development. Thus, adequate planning for an early intervention should involve an accurate forecasting of resources anticipated for implementation: 
I don't think they had the resources when they applied. They had vision and they had desire and they saw a need. But when you apply for Health Canada, those projects, you had better be prepared when you get your money to run with it, because they are fast moving, they are demanding, and you really have to know what you are doing out there. I think they underestimated their resources. (Tr. 5, p.4)

The last first-order category that contributes to the second-order category, Implementing Early Intervention, is Creating Awareness. As a first-order category, Creating Awareness emanates from the raw-data themes that surround the need for expert advocates and public education. In creating awareness for a program that relies heavily on the health-care sector for promotion and delivery, a champion in the form of a physician from the local health-care community is viewed as an important ally to challenge potential biases against early intervention. Thus, the lesson learned in combating negative perceptions of early childhood development programs is that allies from the professions are more likely to influence the opinion of their peers and should be cultivated when implementing early intervention. There is an important relationship between the major dimensions, Challenges to Parenting Plus and Lessons Learned, suggested by the following stakeholders:

... I think some of the key things would be, from my point of view. . we need to have champions. Someone who when all the docs are sitting around, the guy who is going to say what about this and did you know that, have you ever hear of the Perry High School study that showed for every dollar spent on early childhood intervention 6 to 7 dollars can be saved later in remedial education and justice costs and social services. ... we need to have a knowledgeable, well-informed champion who is not afraid to challenge the biases and preconceptions of people who don't really understand what we are trying to achieve and research has shown. (Tr. 18, p. 11)

In addition:

And even the board maybe just a one time session but a real in-depth overview of some research. And I did that but it is better coming from somebody else because somebody further away is usually the expert, seen as the expert. (Tr. 22, p. 6) 
The other raw-data theme for the first-order category, Creating Awareness, pertains to public education about the program in the community. Public education is viewed as integral to the implementation of a community-based early intervention because it engenders community support. The following stakeholder refers to this raw-data theme:

I think maybe more promotion in the community is needed. So that the general community knows the importance of early childhood development and understands what the program is about. And the public, in general, needs more education to make them aware of the program and the need. . .why it is important, and how they can benefit from it. (Tr. 20, p. 10)

The other second-order category from which the major dimension, Lessons Learned, emanates relates to home-visiting benefits. As a second-order category, Home Visiting Benefits emerges from the first-order category Perceived Benefits for Families. Three raw-data themes contribute to this first-order category and include connecting families with resources in the community, being home based, and being a needed service. The first raw-data theme involves the benefits of having a service that enables families to connect with resources, which otherwise might be difficult in a rural health district:

I think as long as we can continue to work and work with them and provide referrals to them, I think there is definitely a need for programs like this in rural Saskatchewan. ... for a lot of families that have special needs or to get to the city for services is really difficult, so it is nice when their services are provided in the community for them. And these are home-based services, too, so that they don't have to worry about transportation, getting out for appointments, and things like that. The workers go right to the home, so that's definitely a benefit. (Tr. 15, p. 4)

The previous quotation also illustrates how connecting with resources is interwoven with the second raw-data theme, which pertains to perceived benefits of a home-based service. This theme is reinforced by the following stakeholder:

It is a program that moves out to the family in its home, where they feel comfortable and where they feel confident. A lot of these people have a lack of self-esteem. And they do not have the strength to move out and really ask for help, so that is really good about the program, that it is home based. (Tr. 20, p. 2) 
The third raw-data theme, building a needed service, is reflected in stakeholders' perception of the home visits as being good for families as depicted by the following quotes:

I don't know the numbers or how many people it affected or anything but I feel that it is a good program in that it helps build positive parent child relationships.

(Tr. 12, p. 4)

Also,

You know I think the clients can only benefit from it, you know the more services we can provide to families at an earlier age the more they benefit. (Tr. 16, p. 2)

The lesson learned, as it relates to home-visiting benefits, is that this form of service delivery is advantageous in a rural health district, where geographical isolation, travel costs, and connecting with community resources have been traditional barriers to accessing needed services for families at psychosocial risk.

Focus Group Results

All parents participating in Parenting Plus received letters of invitation to attend the focus groups. Two focus groups were held at Broadview Hospital on October 24 and November 21,2000, with six people in attendance at each that included four participating parents and two FSWs. An empowerment evaluation model guided data collection, and a four-phase writing process (brainstorming, drafting, revising, and finalizing) characterized the creation of the documents from the focus groups. A recorder took notes, and representative quotations were recorded and reviewed by participants for authenticity at the end of each workshop. Quotations cited in the results are referenced according to the focus group session in which they occurred. For purposes of the evaluation, the focus groups aid in understanding, from the participant's viewpoint, the strengths and 
weaknesses of the program and how it has been operationalized. The focus groups resulted in the creation of a mission statement for the program, the listing and prioritizing of the program's key activities as performed during home visitations, and the establishment of program goals. Once the goals based on the key activities were established, strategies for the attainment of the goals were elicited, as was the documentation that would be required to determine if the goals were obtained.

The development of a mission statement for Parenting Plus served a twofold purpose: It provided a means by which rapport could be established between participants around a common theme, Parenting Plus, and it allowed for the articulation of a vision for Parenting Plus, from the perspective of the participating parents (see Table 4.4). This gave a structure to the rest of the discussion surrounding the program's evaluation.

A list of the key activities that are crucial to the functioning of the program was then generated by focus group participants. The mission statement remained displayed and provided a reference point. Key activities were elicited from the participants during a brainstorming session facilitated by the program evaluator. Participant responses were recorded verbatim and were followed by a group discussion that resulted in a listing of a new key activity. The finalized list of the key activities is reported in Table 4.5.

Once a list of the key activities crucial to the functioning of the program was generated, focus group participants were asked to prioritize the activities. Participants were asked to rate each of the activities at their seat and then to record their activity ratings on a poster sheet. Each activity receives a total and average, which generates a total program rating. Since a brainstorming phase is not required, as activities are already listed in the previous step, the prioritizing stage only went through the drafting, revision, 
and finalizing phases for the two focus groups. The final draft of the program activity ratings is presented in Table 4.6.

Table 4.4

Focus Group Data: Parenting Plus Mission Statement

The mission of Parenting Plus is to provide information and resources to parents about their child's development and how to be a better parent. The purpose of the program is to make parents feel good about themselves, to allow them to know and feel more about themselves and their child. By talking to a support worker, parents are able to realize that feelings both good and bad are alright. In general, the mission of the program is to be non-judgmental and to accept parents and their situations for whom and what they are by providing support to fit the family's needs.

Source: Focus Group Workshop Notes, November 2000.

Table 4.5

Focus Group Data: Parenting Plus Key Activities

1. Assisting parents in recognizing their own strengths.

2. Building confidence, security and trust.

3. Caregiving, childcare, and respite.

4. Learning how to communicate with others (spouse, family members).

5. Interacting with other children in the family.

6. Knowing someone is coming to visit.

7. Emotional support, being a friend and able to phone.

8. Connecting with intersectoral resources.

9. Gift bags and memory books.

10. Goal setting informally and formally.

11. Transportation.

12. Socialization.

13. Providing information on child health and development, early learning, child discipline, safe childcare, and child safety.

Source: Focus Group Workshop Notes, November 2000. 
Table 4.6

Focus Group Data: Parenting Plus Prioritized Program Activity Ratings

\begin{tabular}{|c|c|c|c|c|c|c|c|c|}
\hline \multirow{2}{*}{$\begin{array}{l}\text { Program Activities } \\
\text { Assisting parents in } \\
\text { recognizing strengths }\end{array}$} & \multicolumn{5}{|c|}{ Individual Ratings } & \multicolumn{2}{|c|}{ Average } & \multirow{2}{*}{$\frac{\text { Priority }}{1}$} \\
\hline & 1 & 3 & 1 & 1 & 4 & 5 & 1.8 & \\
\hline Providing information & 2 & 2 & 5 & 2 & 1 & 2 & 2.3 & 2 \\
\hline $\begin{array}{l}\text { Building confidence, } \\
\text { security and trust. }\end{array}$ & 3 & 1 & 6 & 3 & 2 & 5 & 3.3 & 3 \\
\hline Emotional support. & 5 & 4 & 2 & 5 & 6 & 4 & 4.3 & 4 \\
\hline $\begin{array}{l}\text { Connecting with } \\
\text { resources. }\end{array}$ & 4 & 5 & 7 & 4 & 7 & 3 & 5 & 5 \\
\hline $\begin{array}{l}\text { Having a home visit. } \\
\text { (goes with trust) }\end{array}$ & 7 & 7 & 4 & 7 & 3 & 6 & 5.6 & 6 \\
\hline $\begin{array}{l}\text { Learning to } \\
\text { communicate }\end{array}$ & 6 & 6 & 3 & 6 & 8 & 7 & 6 & 7 \\
\hline Transportation & 8 & 8 & 8 & 8 & 5 & 8 & 7.5 & 8 \\
\hline
\end{tabular}

Source: Focus Group Workshop Notes, November 2000.

Participants then listed and established goals based on the program rating activity. Initially, they were asked to establish goals for each activity, however, it was decided by participants to establish two generalized goals and then list the required documentation and strategies necessary to implement the goals with the finalized version reported in Table 4.7. In establishing the goals for the program, the two main topics that emerged during conversation related to instilling knowledge in the family unit and supporting families in recognizing their strengths. Of the two topics identified by the group, developing a statement that would reflect instilling knowledge proved the most difficult. The final version includes three elements that were viewed as key by participants and are associated with program activities: connecting with resources; providing information; and offering emotional support. What is of interest in this goal is the relationship expressed 
by the participants between being able to access information and resources and emotional support. One explanation for this juxtaposition is that when families are overburdened, the provision of information and linkage to resources is indirectly perceived and experienced as a coping resource like emotional support. In articulating the first main goal of the program, the focus group participants also included the required strategies and documentation necessary to attain the goal.

Table 4.7

Focus Group Data: Parenting Plus Goals

\section{Goal \#1.}

To heip support families in recognizing their own strengths and become self-supporting, self sufficient, and self-reliant.

Documentation

Parent completed journal.

Parent completed checklist, ratings, and/or self-evaluation.

Goal setting through family support plans.

Home visit reports, number of visits and length.

Strategies for Goal Attainment

The difference game for goal setting.

Sharing circle, support group, networking and phoning tree.

Role playing, teaching communication skills

Encourage families to access resources.

Build self confidence and self-esteem by accentuating the positives.

Maintaining confidentiality of family members.

\section{Goal \#2}

To instill knowledge by informing families regarding the types of resources available, thereby, enabling healthy families.

\section{Documentation}

Through discussion and questioning, the FSW can create a list of resources and information requested by the parent and provided through Parenting Plus.

\section{Strategies for Goal Attainment}

Create a list of community resources, books, videos, and other (internet).

Contact resources to provide information to the family.

Encourage families to access resources.

Make it fun and activity oriented.

Source: Focus Group Workshop Notes, November 2000. 
The second major goal established by participants related to the support provided families in recognizing and acting on their own strengths and communicating openly. Self-statements were integrated into the goal to describe how families should be supported to become self-supporting, self-sufficient, and self-reliant. As a result, the key activities of the program that were linked to the goal included assisting parents in recognizing their own strengths; supporting family members; and learning to communicate.

\section{Inductive analysis of focus group results}

To aid in the interpretation of the focus group data collected and to be consistent with the results reported for the semi-structured interviews, the general inductive approach to data analysis was used (Thomas, 2000). In conducting an inductive analysis, it was found that the major dimension, Characteristics of Strength-Based Home Visitations, was mentioned by all 6 focus group participants and emerges from 10 raw-data themes, 5 first-order categories, and two second-order categories (see Figure 4.12).

The second-order category, Accessing Resources, is derived from the first-order categories relating to community-based resources and providing information. As a firstorder category, Community-Based Resources emanates from the raw-data themes pertaining to becoming healthy families and intersectoral resources. Becoming healthy families refers to the ability of families to take better care of themselves by being able to access community resources. This is illustrated by the first goal set by families as part of the focus group data collection and is also listed as a key activity. Interestingly, even though focus group participants did not rate connecting with resources as the top activity of the home visitations, they still viewed it as extremely important as shown in Table 4.6. 


$\begin{array}{lll}\text { Raw-Data Themes } & \begin{array}{l}\text { First-Order } \\ \text { Category }\end{array} & \begin{array}{l}\text { Second-Order } \\ \text { Category }\end{array}\end{array}$

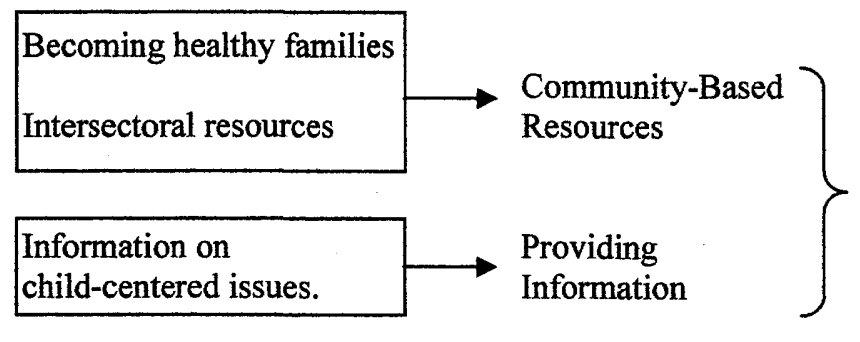

\begin{tabular}{|l|l} 
With family members & \\
& Learning to \\
Communicate
\end{tabular}

Being nonjudgmental
Building confidence,
security and trust.

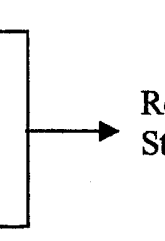

Knowing someone is coming to visit

Socialization

Emotional

Support

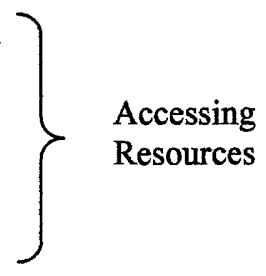

Increasing self-esteem

Figure 4.12. Characteristics of Strength-Based Home Visiting Dimension

When asked to expand on the types of resources that parents benefited from, the list included public- and community-health nurses, local physicians, educators, counselors, and social workers. In terms of the perceived benefits that can be accrued from connecting with resources, the following representative quotations depict how connecting with resources in the community facilitates the development of healthy families:

And when we are given resources or are able to attain resources, then we gain knowledge on how to be better parents and raise healthy families by being more aware of parenting issues. (Focus Group 1) 
Also,

We want to become healthy families, because when parents get the help they need from and through the resources, the return for that is that we are healthier, the family unit is healthy. (Focus Group 1)

The other raw-data theme for the first-order category, Community-Based Resources, is Intersectoral Resources. As a raw-data theme, Intersectoral Resources refers more generally to the variety of resources available in the community. The resources chosen by families reflect their needs and situations at a given time:

Resources . . resources are anything from A-Z. I say I want a speech pathologist, to find, or I may find out that I need a counselor to deal with issues. There are different needs. (Focus Group 1)

The other first-order category, Providing Information, emerges from the raw-data theme that pertains to the provision of information and skill training on child-centered issues. As an activity of the home visitations, providing information and skill training on childcentered issues, such as child development, child health, early learning, child safety, child discipline, and child care, was viewed as being both salient and important to participants in the focus group, as demonstrated by its place as a valued activity in Table 4.6. Moreover, the importance of the relationship between accessing resources and providing information is depicted by the following:

Well that is why I got involved, because I wanted to access other information about other resources, I wanted to be provided with more information. (Focus Group 1)

and

I think resources are very good for us the parents to connect with, because it is difficult to get the best information about resources. (Focus Group 1)

The other second-order category that emerges from the inductive analysis is Developing Well-Being. As a second-order category, Developing Well-Being emanates 
from the three first-order categories that include Learning to Communicate, Recognizing Strengths, and Emotional Support. As a first-order category, Learning to Communicate relates to communications with family members and about oneself. The following illustrates the raw-data theme, communicating about oneself, and how previously-learned closed communication practices can effect a parent's ability to communicate effectively with others:

Some people as they grew up . . were taught not to communicate, were taught not to let their feelings out, were taught that children should be seen and not heard and a lot of people grew up just stuffing this away and are not used to openly talking with others. (Focus Group 2)

The other raw-data theme identified reflects effective communication practices with family members. This is portrayed in the following quotations and illustrates how the desire to enhance one's communication abilities is viewed as a positive way to engage in healthy relationships with family members. Unfortunately, effective communication practices for some program participants are difficult to achieve:

Learning to communicate with others that is important, I just want to learn to communicate more and better, with my family I very seldom say anything. Someone comes over, and I want to be able to communicate better. (Focus Group 2)

Also,

.. I can communicate with my dad but I can't communicate with Larry, like I and my dad live in totally different houses and Larry and I have been living in the same house now for two years and not once have we sat down and had a real conversation like I and my dad. Like I don't understand why Larry and I can't communicate without getting into an argument. (Focus Group 2)

The other first-order category that results in the emergence of the second-order category, Developing Well-Being, is Recognizing Strengths. As a first-order category, Recognizing Strengths emerges from the raw-data themes that include being nonjudgmental and building confidence, security, and trust. The importance of 
recognizing families' strengths to enable them to become self-reliant, self-sufficient, and self-supporting is depicted in the first program goal articulated by parents as reported in Table 4.7. Moreover, the importance of being nonjudgmental and how it is interwoven with emotional support and helping families in recognizing their own strengths is aptly illustrated by the following:

. . .we are all worth it, because there are a lot of feelings of worthlessness out there when you have a child and you are in a relationship and you are not happy with it and all those things. Having these guys come and talk to you and come into your house, it just helps you. They may send you on the right road to get out of the relationship if it is the right thing for you. They may let you know that there is somebody to lean on. If you are in those sort of situations, there is no judgment.

(Focus Group 1)

Further:

For us, we are allowed to have whatever feelings we feel, and we are provided with that support to say it is okay to have those feelings. (Focus Group 1)

The final first-order category for the second-order category, Developing Well-Being, is Emotional Support. As a first-order category, Emotional Support relates to the raw-data themes that include knowing someone is coming to visit, socialization, and increasing self-esteem. The relationship between the raw-data themes increasing self-esteem and the security in expecting a home visitor is aptly depicted in the following quote:

By accentuating the positive, building self-confidence and self-esteem and security, this is important for a new mom, knowing that someone is coming to see you. You don't know how important this is to a new mom. (Focus Group 1)

Providing emotional support is articulated as a salient and necessary goal for the program, as reported in Table 4.7. Furthermore, having a home visit and emotional support were prioritized as important activities by participants, as illustrated in Table 4.6. The relationship between the raw-data theme increasing self-esteem and emotional support is portrayed by the following participant: 
We would work together and look at strengths to increase self-esteem in order to feel self-reliant and self-sufficient. . . .You have to feel good about yourself and that is increasing ones self-esteem and that is important in what we do during a home visit.

(Focus Group 2)

Thus, the results from the focus group reflect the most salient features of the paraprofessional home-visiting activities that occur in Parenting Plus from the parent's perspective. The parents spoke positively about paraprofessional home-visiting and were receptive to the program. These results confirm that a strength-based approach is being utilized and is viewed as an important feature to those families served. Within this context, it would appear that accessing resources and developing the well-being of families are features of the program that tend to predominate its operationalization, as determined by an inductive analysis of the focus group data. As well, parents informally spoke about how paraprofessionals chosen from the community are an integral component to the program as they aid in engaging and maintaining families. This was especially important to First Nation focus group participants. As focus group participants have shown, the extent to which families develop trust and engage in program delivery is an important consideration that needs be addressed in the broader context of homevisitations and the models of service delivery practiced.

\section{Family-Centered Program Rating Scale Results}

In order to ascertain the strengths and weaknesses of the program as reported by the participating parents while at the same time aid in the understanding of the program model that has emerged and been operationalized, the Family-Centered Program Rating Scale developed by Murphy et al. (1991) was administered. By using a quantitative method, multiple data sources achieve results for the research questions posited that aid 
in triangulation. As well, the FamPRS was slightly modified to better reflect the focus group data collected surrounding the evaluation of Parenting Plus. These modifications mainly pertained to items which referred to "disability", as this was considered a pejorative term. This resulted in changing the wording of six items, as previously reported.

Due to the highly transient nature of the families involved in Parenting Plus and the difficulties in contacting parents at their homes (many are without telephones), it was decided not to collect data for the rating scale through the mail or by a phone call. As a result, parents were given the opportunity to complete the parent's version of the FamPRS when visited by the FSW during December 2000. Eleven parent-completed FamPRS were collected by the FSWs with two parents declining to participate and five unable to be contacted. Parenting Plus staff then mailed the FamPRS and the principal investigator attempted to make contact over the telephone, however, no additional FamPRS were completed.

Consequently, not all parents involved in Parenting Plus completed the FamPRS, which makes the generalization of the results to all participants not possible. Nevertheless, the FamPRS was developed as an evaluation tool for early childhood development programs and provides information about the degree to which the program implemented and in operation is family centered. For the purposes of the current evaluation, the results from the FamPRS aid in determining from the parent's perspective the strengths and weaknesses of the program and what model of service delivery has emerged and been operationalized. In this respect, the FamPRS provides ratings of program performance and importance of program features. The ratings obtained on the 
FamPRS are derived from the following subscales (Murphy \& Lee, 1992):

1. Flexibility and innovation in programming;

2. Providing and co-ordinating responsive services;

3. Individualizing services and ways of handling complaints;

4. Providing appropriate and timely information;

5. Communication and timing;

6. Developing and maintaining comfortable relationships;

7. Building family-staff collaboration;

8. Respecting the family as decision-maker;

9. Respecting the family's expertise and areas of strength;

10. Recognizing the family's needs for autonomy; and

11. Building positive expectations. (p. 34-35)

In interpreting the FamPRS, average group scores can be used as indicators of a program's performance and importance of the eleven different program practices. When scoring the FamPRS, individual item responses are tallied and importance and performance averages for each item are determined. For each item, there are four possible responses presented on a four point likert scale. For example, an item rated as "not important" would receive a score of 1 , "somewhat important" a score of 2 , "important" a score of 3 , while an item rated as "very important" would receive a score of 4 . Item averages are then transferred to a group scoring form that is used to calculate the 11 subscale averages that are graphed and reported in Figure 4.13. Since the purpose of the FamPRS is to provide information pertaining to a program and not an individual family member, only subscale averages are reported as recommended in the program manual (Murphy \& Lee, 1992). 


\section{$\square$ Performance $\square$ Importance}

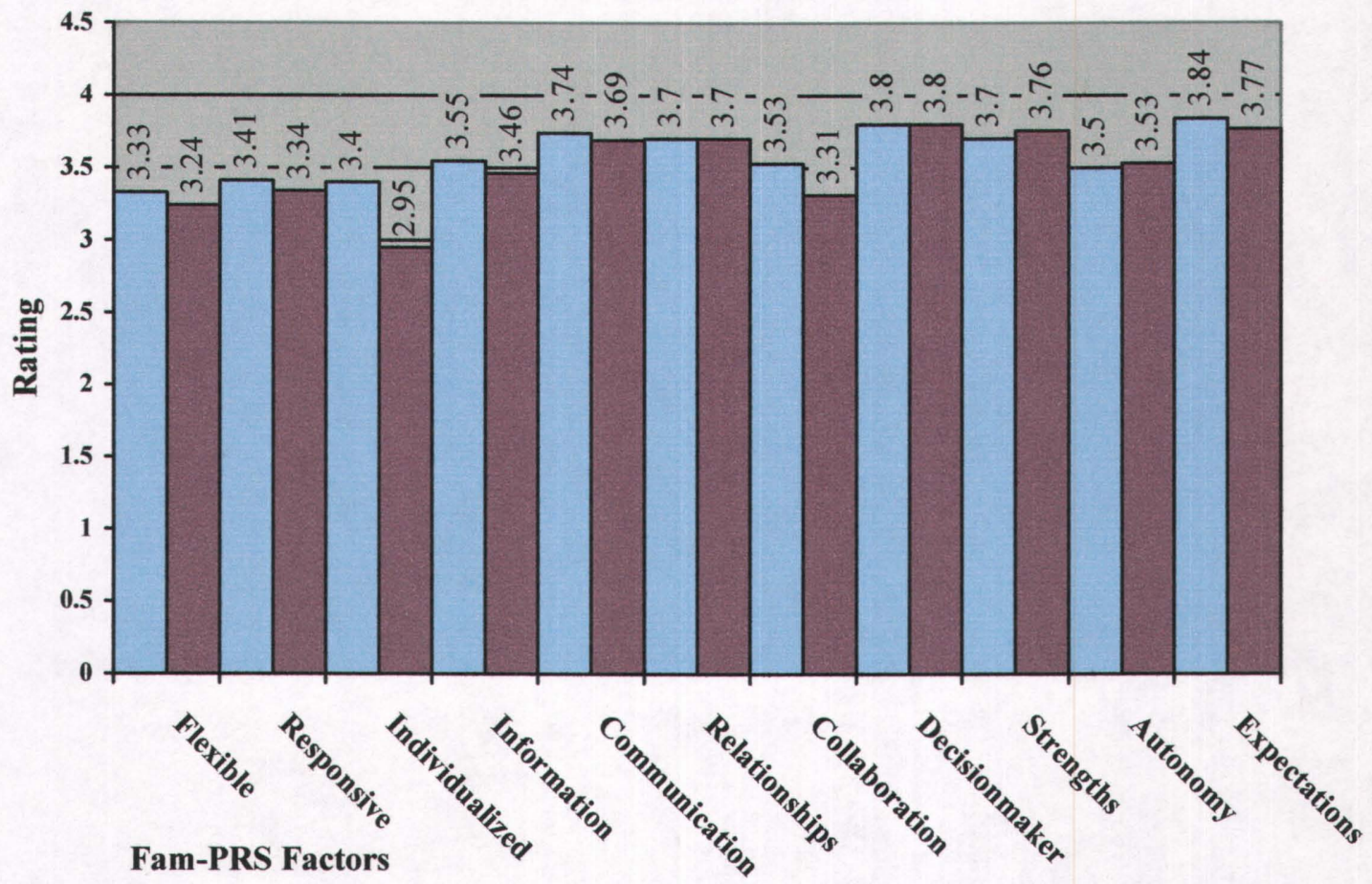

Figure 4.13. Family-Centered Program Rating Scale Results

In interpreting the results, what is of interest is that, for all subscales, program performance was either equal or better than importance ratings for the same subscales. This is a significant result because it indicates areas in the program that are already meeting or exceeding the needs and wishes of the parents. Areas of strength for Parenting Plus as determined by the FamPRS are found in developing and maintaining comfortable relationships, respecting the family as decision-maker, respecting the family's expertise and strengths, and building positive expectations. The least important feature of the program, but one in which Parenting Plus did well, was individualizing services and ways of handling complaints. These results are expected, considering previous results reported 
for the focus groups, and are congruent with the strength-based model implemented in Parenting Plus. From these preliminary results, it would appear that the program is operating from a strength-based system of program delivery either meeting or exceeding the expectations and needs of parents involved and who completed the FamPRS.

Anecdotal comments were also elicited from the parents through two open ended questions at the end of the rating scale. The comments provided by the parents are displayed below for each of the questions (see Table 4.8). It is not known if the parents who did not complete the FamPRS have similar perceptions or experiences of Parenting Plus as those who did. Nevertheless, it would appear that those parents who completed the FamPRS are very satisfied with the service delivery presently being provided. Of interest in the anecdotal remarks, as they relate to program delivery, is the need by some parents to have established parent groups that could meet on a regular basis.

Table 4.8

\section{FamPRS Anecdotal Comments}

Question 1.

What things about Parenting Plus make it especially helpful and welcoming?

Well its helping my little family by being there for us, by letting us know about how to raise my first child the right way as he grows up.

They're doing a good job. Support, immediate response, it is all good.

Building confidence in parenting and in ourselves. What a wonderful feeling! Thank-you!

Having a home visitor and baby bags.

Home visits, providing information, putting me in contact with professionals.

Staff is supportive, friendly, very helpful in making goals. Gift bags, binders, and pictures.

Question 2.

What are the ways that Parenting Plus could be more helpful and welcoming to your family?

Parent groups, social times, play times.

Welcoming tea, social get-together. 


\section{Summary}

The four major components of Parenting Plus derived from the Pipestone Health District's Health Transition Fund proposal (1998) can provide a useful and important framework to synthesize the results. To contextualize Parenting Plus within an integrated framework, the program's proposed components are used as basis for understanding. In this respect, the components of the program include: screening and assessment; homevisitations; informal community support; and co-ordination of services and supports. Although the components are presented independently, they are integrated within the operationalization of the program.

\section{Screening and Assessment}

Although initial difficulties in obtaining screens resulted in the program operating under capacity, recent changes have proved positive and have benefited its growth and development. Renewed partnership building between Parenting Plus and other health districts, hospitals, First Nations, family doctors, and public- and community-health nurses has eliminated some of the barriers to implementation that Parenting Plus initially experienced in obtaining medical screens. As a result, a more systematic screening process is in place, resulting in a higher number of positive screens, and ultimately, a larger number of parents participating in the program. This has resulted in Parenting Plus operating at near capacity for its first year of operation. This is despite the fact that screening numbers are compromised due to agreements with First Nations that also compromise the delivery of creative outreach services to these same communities.

Nevertheless, from the results of the document review and semi-structured interviews, 
it would appear that Parenting Plus is reaching its intended target audience. Parents participating in the program are young, single mothers, the majority of whom are unemployed and have less than a Grade 12 education. In terms of a systematic screening process, this is an important finding because it indicates efforts to promote the program should be directed towards this target group. It is believed that entry into the program should not be restricted to birth mothers with children from birth to three months but ought to be expanded so that parents who might not immediately see an advantage to being in the program would still have the opportunity to do so at a later date.

However, deviations from the program protocol as it pertains to screening have proved problematic. Although the program model, Hawaii Healthy Start, calls for screens to be completed while the mother is in the hospital, interview results indicate that such a procedure was found unworkable. This was due to difficulties in obtaining information from the birth mother during her relatively brief stay in the hospital. Consequently, medical record screens are referred by local physicians, public-health nurses, communityhealth nurses, local hospitals, and Regina General Hospital to the Parenting Plus coordinator. A negative effect of all screening and referrals being conducted through Parenting Plus is that increased responsibilities and demands are being placed on the program co-ordinator that were not anticipated during program planning. Nevertheless, benefits are found in a more systematic and complete screening that has resulted in an increased number of parents being screened. In turn, the base rates for Parenting Plus are now comparable to Hawaii Healthy Start population projections.

Although the data is specific to the sample under consideration, assessment for psychosocial risk seems to be largely determined by interviews with the mother. 
According to program protocol, a score $>25$ on the Family Stress Checklist (1976) by either parent is used as a clinical cut-off to determine if the assessment is positive and if the family is to be offered home visits. However, few fathers are present at the assessment, and items for the father are derived through conversation with the mother or are unknown. As well, when reviewing the risk factors that present themselves during assessment, it is found that very few parents self-disclose surrounding child abuse and neglect or harsh disciplining practices.

\section{Home Visitations}

Participant mobility and program staff travel costs continue to be major challenges that face the delivery of the program in a rural health district, as reported in the interview results. In the document review, FSWs were found to have attempted home visits ranging from 1 to 6 visits, with an average of 1.18 missed visits per family. Moreover, continuity of service delivery as it pertains to home visitations proved problematic when families left the health district and could not be followed up. Family support workers reported having difficulty in re-engaging families upon their return when the time away from the community was extensive. As well, the added travel costs incurred in a rural health district with a transient target population were not anticipated in program design and planning. Thus, implementing a program protocol similar to Hawaii Healthy Start that was developed as a statewide system of support and delivered in a dense urban geographical environment has proven problematic.

In terms of the demographics of the current caseload, young, single mothers with less than Grade 12 are more likely to accept home visitations. This is not surprising as young, 
single parents are less prepared to undertake child rearing and might see greater benefit in relating with paraprofessionals. In terms of Parenting Plus's current program delivery, this finding might have implications for the types of home visits conducted and, ultimately, the information and resources provided, although further research will need to explore this area in more depth.

Case conferences with FSWs and the program co-ordinator continue to be held on a regular basis and provide a means by which decisions regarding engagement, trust building, and goal setting for families can be discussed. At the time of evaluation, as would be expected, the frequency and duration of home visitations for parents newly enrolled in the program is relatively high in comparison to parents who have been in the program for four to six months. However, as more families enter the program and progress through the level system, the need to develop individual family support plans will predominate.

Results from the FamPRS completed by program participants indicate that areas of strength for the program are found in developing and maintaining comfortable relationships, respecting the family as decision maker, respecting the family's expertise and strengths, and building positive expectations. Similar results are found for the focus groups. The key activities of home visitations identified by parents include: assisting parents in recognizing their own strengths; building confidence, security, and trust; providing emotional support; connecting with resources; and providing information on child health and development. Moreover, in defining a vision or mission statement for Parenting Plus, participants articulated and reinforced the non-judgmental, accepting, and supportive nature of the home visits provided. The inductive analysis of the focus group 
data revealed that the major dimension, Characteristics of Strength-Based Home Visiting, emerged from Accessing Resources and Developing Well-Being. Thus, the program implemented and now in operation corresponds very closely with the components of the Hawaii Healthy Start model that include: providing supportive services in parent skill building; emotional support; information about child care; and linkages to intersectoral resources. According to FamPRS results, the service delivery provided by Parenting Plus meets or exceeds parent's expectations and is viewed positively by participants.

One of the barriers that remains for program staff, as determined by the semistructured interviews, is access to training modules that are presently not being delivered in Saskatchewan. As well, it is recommended that the program implement a system for monitoring short- and long-term outcomes to gauge the program's effectiveness. This is viewed as essential if negative perceptions surrounding early intervention are to be eradicated. Participants in the focus groups felt such a tracking system would provide a means to ascertain if they were recognizing their own strengths and becoming selfsufficient and self-reliant, as articulated in the first program goal. Moreover, parents in defining the strategies that can best be used by paraprofessionals suggested there was a need for more interactions like role plays, sharing circles, and support groups.

\section{Linkages between Family and Health Care Community}

The third program component relates to the establishment and maintenance of linkages between the family and their medical care home facility. For Parenting Plus, the family's medical care provider would be the local physician, public-health nurse, or community-health nurse. In the focus groups, parents reported that establishing linkages 
to intersectoral resources like public-health nurses, community-health nurses, local physicians, mental-heath professionals, social workers, and educators was a significant feature of the home visitations. Thus, they believed that having a link to medical services for families is basic to the health and well-being of their children. However, even though information was provided on how to access services and whom to contact regarding child and family health and well-being, there was infrequent communication and feedback between FSWs and health care practitioners concerning families being served.

Thus, from the perspective of an implementation evaluation, it was found that the establishment of linkages for families are enhanced when there are collaborative structures in place that facilitate the integration of intersectoral resources. Unfortunately, Parenting Plus experienced some initial difficulties in partnership building with health care practitioners. Not only did this challenge the screening and promotion of the program locally, but it also affected the co-ordination of services between Parenting Plus and health care practitioners. Linkages with health care practitioners for families would be augmented if there were structures in place that facilitated the intersectoral coordination of services between Parenting Plus and medical care services. To help meet this challenge, Parenting Plus has begun to provide screening and assessment statistics to health care practitioners and communicate informally regarding families being served.

However, the program has met resistance from some sectors. Specifically, health care practitioners would like to see an increased role by public health in program delivery, particularly as it refers to health education. Furthermore, health care practitioners questioned the requisite skills of the FSW to perform the job. Support of the program by some stakeholders was found wanting. However, as Parenting Plus has evolved, 
increased efforts have been directed towards the establishment of linkages, not only on the pragmatic level of home visitations, but through the development of mechanisms for the co-ordination of services and supports.

\section{Co-ordination of Services and Supports}

The fourth program component of the model seeks to establish and maintain referrals to and co-ordination with community services for families. Like the third component, this feature of the program model is intended to address the multifaceted needs of families who are overburdened. The distinction, from a program delivery standpoint, is that this fourth component is not specific to health care practitioners but can include other resources, like social workers, employment agencies, and educational institutions. Parents participating in the focus groups and FSWs being interviewed both saw that the linking of resources should include not only health care practitioners but local community resources, as well.

In developing strategies by which Parenting Plus could help parents connect to available resources, the creation of a community resources index was suggested by participants in the focus groups. To this end, Parenting Plus staff have created a resource brochure that assists families in connecting with resources in their community. However, parents participating in the focus group would also like to see more contact with resources, either individually or through the provision of information sessions. Stakeholders believed one of the major benefits to the provision of home-visitation services in rural health districts is in the ability to link families with resources that otherwise might not be accessible. From the perspective of program delivery, one of the 
challenges that faced Parenting Plus was providing home visitations and linking to resources when families moved out of the district.

To facilitate the co-ordination and referral of community supports that is formalized in the governance structure of the program, the protocol called for the establishment of an advisory council. Unfortunately, due to the lack of time for implementation, the development of participatory structures like an advisory council was neglected. In recognition of this shortcoming, Parenting Plus has sought out local participation from intersectoral agencies in the Pipestone Health District that are committed to the betterment of children and their families. This initiative has been warmly received by stakeholders. Not only does an advisory council formalize the role of intersectoral service agencies within the hierarchy of Parenting Plus, it also provides a forum for input and discussion and promotes the program locally. The establishment of participatory structures like the advisory council facilitates the opportunities for co-ordination of services and supports from a service delivery perspective.

Thus, it was found that intersectoral involvement is crucial to any pilot project's success. Timelines need to take allow for partnership building with stakeholders and the public prior program implementation. Furthermore, public education and awareness surrounding the program model's feasibility and applicability prior to implementation would aid in alleviating misconceptions and misunderstandings. This in turn, would build partnerships thereby facilitating program development and implementation among stakeholders. 


\section{CHAPTER FIVE}

\section{Discussion}

\section{Overview}

Society is undergoing a period of increasing stress. The expansion of the market economy in North America has left us particularly susceptible to rapid social breakdown, resulting in increased apathy, alienation, delinquency, and violence. The effect of material factors, social support, and psychosocial conditions on the health of the individual and family has been well documented.

However, the deleterious consequences of these stressors are not uniformly felt throughout society. The ability to provide access to services and acceptable living conditions varies considerably among families, in Canada, to a large degree because of the inequality of incomes. As public services are cut back, the hardship for low-income families increases. The pervasive nature of social change has left even families with moderate economic resources experiencing difficulty providing adequately for the developmental needs of their children and according to Keating and Mustard:

. . during periods of profound social change, such as the present, some sectors of society are at high risk of encountering a decline of social support and hence an inadequate nurturing of developmental needs. Families with young children are often the most vulnerable, and this appears to be true in our contemporary society. Although economically poor families are at the highest risk for this form of family insecurity, the changes we are experiencing are so widespread that negative consequences are occurring even for the children of families that are moderately secure economically. In particular, labour market policies that do not recognize the extensive demands placed on families with young children, combined with the dearth of good, affordable childcare, create a situation in which adequate nurturing of the next generation cannot be assured. (p. 88) 
To address these needs in Canada, there have been increased efforts in both the public and private sectors towards the development and implementation of early intervention programs. Non-profit organizations and the federal and provincial governments have figured prominently in forging partnerships to develop a system of service delivery. As early intervention continues to be a priority on the provincial and national scenes, the need for reliable and sustainable programs increases. Moreover, these efforts continue to draw upon intersectoral resources in the community to address the multifaceted and complex needs of families and their children. As a result, there has been a growing demand for good evaluations that inform subsequent efforts and build a knowledge base that can guide program development in early intervention. The present evaluation is one such example.

As an applied research study of Parenting Plus, a utilization-focused evaluation involving stakeholders that follows an implementation framework was designed. The objectives of the study were threefold. The first goal was to understand the model of service delivery that has emerged and been operationalized for Parenting Plus. The second aim was to explore the characteristics of the implementation process that have facilitated or hindered the development of the program. The third intention was to gain insight into what parents experience and understand about the paraprofessional home visitations they have received from Parenting Plus. To help integrate and synthesize the objectives of the evaluation a conceptual model of service delivery for Parenting Plus and one of effective practices for intersectoral programs like Parenting Plus are developed. 


\section{Findings}

To demonstrate how Parenting Plus has been operationalized a model of the service delivery at the time of the study is depicted in Figure 5.1. The components of the program include: systematic screening and assessment, the provision of paraprofessional home visitations, and accessing intersectoral resources by connecting families to those assets most commonly available in the community.

As the model illustrates, by following a two-stage screening and assessment process, of those families that are assessed at high risk, two possible service delivery options exist. Either the family directly enrolls in the home visitations, or creative outreach is offered. If the family accepts creative outreach, the Family Support Worker (FSW) maintains contact with the family for a three-month period at the end of which the family has the option to enroll in the home visitations.

Once a family accepts home visitations, the FSW attempts to engage and establish rapport with the family. The home visitations offered provide individualized services to maintain the health and well-being of the family through therapeutic support. This involves the establishment of an empathetic relationship which allows the client the opportunity to express personal thoughts and feelings. The key features of the home visitations according to the participants include: (a) teaching communication skills, (b) helping families recognize their strengths, (c) providing child-centered information, (d) offering emotional support, (e) building confidence and trust, (f) providing respite and child care; and (g) accessing intersectoral resources. The last feature of the service delivery model involves making intersectoral resources available. This can occur either 


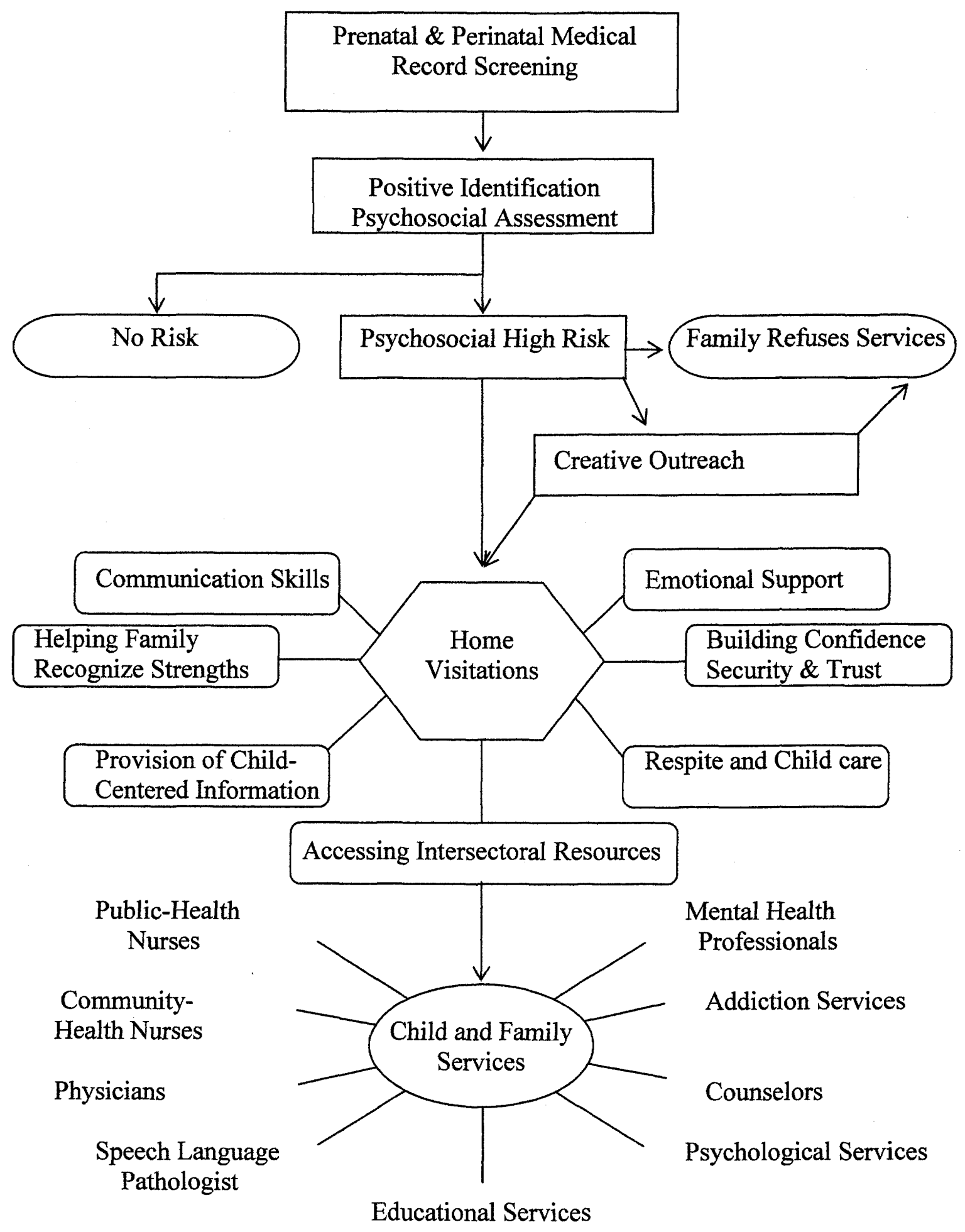

Figure 5.1 Parenting Plus Model of Service Delivery 
formally or informally. For the benefit of the family being served, the FSW attempts to establish linkages with community-based services such as: (a) public-health nurses, (b) community-health nurses, (c) physicians, (d) speech language pathologists, (e) educational services, (f) psychological services, (g) counselors, (h) addiction services, and (i) mental health professionals.

In light of the model described, the major dimensions developed from the general inductive approach will be used so the findings can be explicated and developed, as advocated by Thomas (2000). Although, the major dimensions that resulted from the data analysis have been reported and illustrated with relevant quotations by stakeholders in the results, the following discussion will integrate literature from the research to elaborate on similarities and dissimilarities between the current findings and those otherwise reported. The headings used to organize the findings of the present study that are developed from the major dimensions include: Effective Practices, Screening and Assessment, Sustainability, and Strength-Based Home Visitations.

\section{Effective Practices}

The first major finding shows that ineffective partnership building and communication during the implementation of Parenting Plus challenged its development. Specifically, the interviews reveal a wide range of obstacles that directly relate to unsuccessful communication practices and the failure to form alliances. Difficulties in establishing agreements with intersectoral stakeholders for the provision of screens during program implementation resulted in lower than anticipated enrollment in the program initially. From a program development perspective, these findings are 
significant. They underscore the value of developing participatory structures prior to the launching of new programs as revealed in the Lessons Learned dimension. Building community readiness, consulting and collaborating with stakeholders, as well as formalizing the role of stakeholders through an advisory council are valuable activities that should be undertaken prior to the launching of new programs. This is integral when intersectoral resources are involved in either the planning or implementation of the program. Furthermore, Saskatchewan's Action Plan for Children calls for full partnership to provide a co-ordinated and integrated system of service delivery (Government of Saskatchewan, 1993). Thus, community-based services that provide a seamless system of support are needed for the effective practice of early intervention with the role of the school in Saskatchewan being expanded to provide pre-Kindergarten programs (Government of Saskatchewan, 2001).

Second, from a resource-based perspective, creating partnerships is important because it aids in building community capacity (Trivette \& Dunst, 2000). Focus group participants acknowledged that one of the key activities of the home visitations related to accessing community-based resources and providing information on child-centered practices to promote the development of healthy families. Thus, building community capacity is viewed as an integral component in resource-based intervention practices, like Parenting Plus. The methods used to build community capacity according to Dunst and Trivette include:

1. Identifying strengths of community people and groups;

2. Demonstrating how these strengths address child and family desires; and

3. Eliminating barriers through use of other resources. (p. 86) 
Clearly, challenges to the implementation of Parenting Plus arose from difficulties, initially, to forge partnerships with intersectoral stakeholders. If the purpose of building community capacity is to recognize the strengths and assets of a group of people and by so doing enhance family accessibility to resources, then it would follow that collaborative intersectoral relationships between stakeholders is desirable.

What distinguishes resource-based interventions and those that target specific stressors, such as Guralnick's risk factors model $(1997,1998)$, is that the resource-based model attempts to draw on the family's ability to access opportunities within the community. Although Dunst and Trivette (1997) acknowledge that families do and can influence child outcomes, the purpose of the intervention is to mediate the provision of resources and supports so that child learning and development are enhanced. Moreover, as the third component of Parenting Plus relates to the establishment and maintenance of linkages between the family and community supports, it is imperative that mechanisms for feedback be established. Lack of feedback among stakeholders who were health-care providers in the Pipestone Health District was a disadvantage to the implementation of the program. What the building partnership and communication findings indicate, then, is that when participatory structures and partnerships are not formed with intersectoral stakeholders initially, the implementation of a new program, especially one that is strength-based, can be severely compromised, and the family's ability to maximize benefits from home visitations is jeopardized.

To help conceptualize the effective practices relevant to the development and implementation of an intersectoral program, a model for understanding is depicted. As is illustrated in Figure 5.2 a three-phase process for program implementation that includes 
program development planning, pre-program implementation, and program implementation is advocated. Within each phase are actions for effective practices and the resultant impact of such actions on the program. From an implementation perspective, the practices articulated are based on what is deemed most important to the development of an intersectoral program as revealed in the present study.

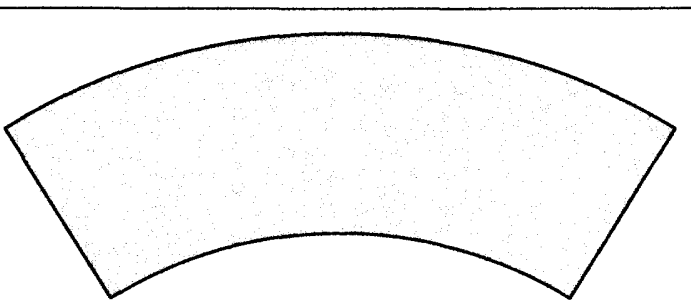

PHASE 2

Pre-Program Implementation

ACTION

Public relations campaign regarding efficacy of program. Cultivate stakeholder advocates to promote program locally thru professional and public education seminars \& the media.

\section{IMPACT}

Secures community readiness for program implementation.
PHASE 3

Program Implementation

ACTION

Establish and provide linkages for feedback to funding agency, stakeholders impacted, and community. Monitor program and address implementation challenges.

\section{IMPACT}

Short cycle decisions, optimally, leading to program stabilization.

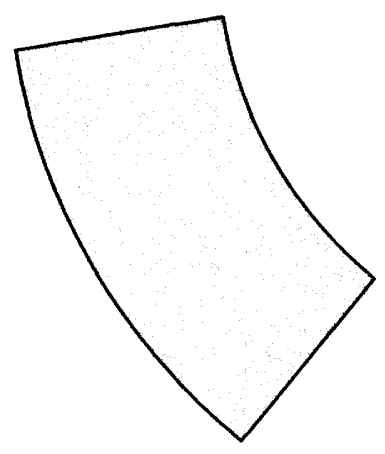

PHASE 1

Program Development Planning

ACTION

Identify stakeholders effected by program.

Establish stakeholder advisory council.

IMPACT

Provides forum for input into program

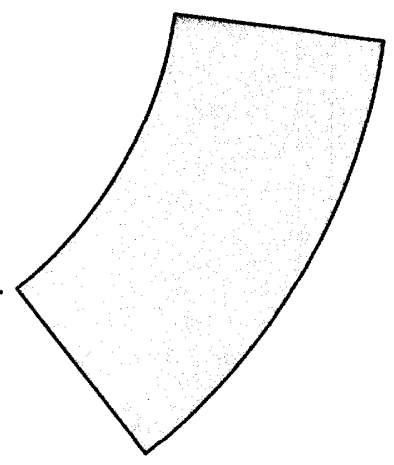

development, builds partnerships, and

establishes mechanisms for stakeholder

involvement in program governance structure.

Figure 5.2 Effective Practices for Intersectoral Program Implementation 
Salient features of the model relate to the need for the establishment of an advisory council that has input into program development planning from the outset. As the first phase of intersectoral program implementation, the identification of stakeholders and their formal involvement in the governance structure of a newly planned program is viewed as essential if partnerships are to be established at the intersectoral level. From a resource-based perspective this is important because it begins to formalize some of the existing informal linkages that might have already developed to accommodate service delivery.

The second phase of the model pertains to pre-program implementation. This phase is characterized by public relations initiatives that inform community members and professionals alike regarding the efficacy of a particular program. The pre-program implementation phase is also viewed as occurring after program development planning. The rationale for such a decision is relatively straightforward as the pre-program implementation phase is the vehicle by which the dissemination of information outside of the advisory council occurs. Program staff need to have highly developed communication skills as public education seminars and the cultivation of stakeholder allies who would advocate for the program's implementation are viewed as essential and necessary to creating community readiness for a newly developed intersectoral program.

The third phase of the model involves program implementation and is characterized by the establishment of feedback linkages to the funding agency, intersectoral stakeholders, and the community. Furthermore, every effort should be made to monitor program implementation so that challenges to the implementation process are addressed. The fidelity of the program to the model operationalized and characteristics of the 
implementation process can guide evaluation efforts. Optimally, this would lead to short cycle decisions that would better inform program implementation and lead to program stabilization. Thus, by following the three-phase process, effective practices for intersectoral program implementation are facilitated.

\section{Screening and Assessment}

As a result of a more systematic screening and assessment process, enrollment in Parenting Plus is comparable to the predicted base rates as articulated in the Hawaii Healthy Start program manual (Hawaii Department of Health, Maternal and Child Health Branch, 1994b). Although a number of procedural difficulties relating to the implementation of the model arose, the program was able to adapt the protocol to fit local needs. Most notably, ethical concerns were an initial impediment to the provision of the screens. This was due in part to difficulties in partnership building and communication. However, other researchers have also questioned the logic of the Hawaii Healthy Start model and the apparent ambiguity and failure to explain how program staff should handle ethical concerns relating to the procurement and completion of the screens (Wallach \& Lister, 1995).

There are a number of important findings that can be drawn from the present study that are relevant to targeted interventions. First, stakeholders questioned the ability of the screen to identify correctly the target population. Does the screen accurately select families at-risk? Do the risk factors because of their inclusion on the screen act as good predictors of psychosocial adjustment? What is the potential for variability of risk status? The findings reported for the present study support research conducted by Offord, 
Kraemer, Kazdin, Jensen, Harrington, and Gardner (1999) and Guterman (1999) on targeted approaches to early intervention. According to Offord et al., there are a number of disadvantages to the targeted approach. The three relevant to the present discussion are the boundary problem, the stability of risk status, and the inability to target accurately.

The boundary or threshold effect occurs when there are disparities in regard to the cutoffs used in individual assessments. Interview results reported for Parenting Plus indicate that stakeholders believe there was the potential for a group of people to be missed by the screening process. This was expressed in the raw data theme that related to the need for a continuum of services so those who might not be identified at high risk would still be able to access home visitations and in the raw data theme that pertained to the ability of the screen to accurately identify psychosocial risk.

The stability of the screen refers to the potential variability of risk status over time. For example, if risk status changes from year to year, then repeated screens would be necessary. Because of this, the fixed markers used on a screen are of particular importance (Offord et al.). A parallel finding in the present study is the need to expand the time frame for acceptance into the program. The birth to three-month period is not necessarily a time of perceived stress by the family. Thus, risk status determined during this time might reflect a "honeymoon" period. Consequently, there is the potential for the screen and assessment process to inaccurately reflect a family's risk status and the possibility that families would refuse services because of perceived low risk. This variability in risk status is a major deterrent for targeted interventions because there is the potential for the positive predictive value and the negative predictive value of the screen to be compromised (Offord et al.). This parallels what Guterman found in his meta- 
analytic study of population-based and targeted home-visitation programs to prevent child abuse and neglect. According to Guterman:

Findings suggest that psychosocial risk screens conducted at a single point near birth may tap family variables that are less stable over time than demographic realities in their relation to child maltreatment. (p. 875)

The third finding reported by Offord et al. (1999) for targeted interventions that parallels the present study relates to the inability of the screen to accurately identify families at-risk. According to Offord et al., one of the major disadvantages of the targeted approach is found in its inability to predict future behaviors based on screening results. They find there is the potential to either enroll families that are less amenable to change or screen out families that are more amenable to change. A similar concern is expressed in the present study by stakeholders who questioned the ability of the medical record screen to accurately identify present and forecast future psychosocial adjustment.

\section{Sustainability}

Findings from the present study show that the mobility of participants and the retention of families in rural or geographically remote health districts will create ongoing challenges to the sustainability of the Hawaii Healthy Start model. Specifically, the document review revealed that FSWs spend a great deal of time attempting to contact families at home or on the telephone so as to engage them in home visitations. Parenting Plus aggressively pursues over one-third of the families who are on creative outreach to reduce the program's attrition rate. These findings are further reinforced in the interviews, which reveal that participant mobility, the associated staff travel costs, and the time involved trying to engage some families creates obstacles. The difficulties 
practitioners have in attracting and retaining families at-risk who receive home visitations are well documented in the literature (Britt, 1998; Duggan, McFarlane et al., 1999; Kitzman, Olds, et al., 1997). Findings reported by Duggan, McFarlane et al. indicate attrition is a common problem in Hawaii Healthy Start. Of the programs evaluated, it was found that a number of families left the program because they no longer reside in the catchments served. This led Duggan, McFarlane et al. to conclude that if service availability is expanded, the attrition rate would be reduced. Moreover, Duggan, McFarlane et al. believe that this is not unexpected when screening is used as a method to identify and enroll families. Rather, if families self enroll or request services, the probability of the family dropping out of the program is reduced (Duggan, McFarlane et al.).

The other major finding of the present study that relates to program sustainability is paraprofessional knowledgeableness. Many of the stakeholders-believe the paraprofessionals lacked the formal training necessary to establish credibility. This finding is not surprising and parallels work conducted by Hiatt, Sampson, and Baird (1997) who compared nurse and paraprofessional home visitations and found that "given their often limited formal education and training, paraprofessionals encountered questions about their ability from established service professionals" (p. 81). As a result, Hiatt et al. reported that paraprofessionals also drew resistance when they were trying to obtain or relay information regarding the families they were serving during home visitations to health care professionals. This in part might explain why in the present study there is a lack of feedback and role clarity between the FSWs and other health care professionals. Thus, because paraprofessionals lack credibility among their professional counterparts 
the potential to establish linkages for feedback is compromised that when coupled with participant mobility can and does affect a program's sustainability.

\section{Strength-Based Home Visitations}

The next finding for the present study relates to how a strength-based approach to family-centered early intervention is characteristic of the home visitations. In a review of early interventions that offer family-centered home visits, Trivette and Dunst (2000) organize 17 family-centered practices into four major themes. According to Trivette and Dunst, the themes that encapsulate best practices for family-based early interventions include:

1. families and professionals share responsibility and work collaboratively;

2. practices strengthen family functioning;

3. practices are individualized and flexible; and

4. practices are strengths- and asset-based. (pp. 45-46)

Dunst and Trivette further elaborate on these themes by listing the recommended practices for family-centered interventions appropriate to each category.

For Parenting Plus, what participants' value most about the home visitations are relationship building and helping families identify their strengths. Findings of the present study from the FamPRS, which is an instrument designed to evaluate family-centered practices, indicate that areas of the home visitations being provided that meet or exceed the needs or wishes of the parents include: (a) developing and maintaining comfortable relationships with the family, (b) respecting the family as decision-maker, (c) respecting the family's expertise and strengths, and (d) building positive expectations. Similarly, findings from the inductive analysis of focus group results confirm and elaborate on the FamPRS findings. Focus group findings of the characteristics of the strength-based home 
visitations provided by Parenting Plus include: (a) providing emotional support, (b) assisting parents in recognizing strengths, (c) helping parents learn to communicate more effectively, (d) providing information pertaining to child-centered issues, and (e) helping families access community-based resources. Thus, it would appear that elements of the home visitations pertaining to relationship building and strengthening families are the most salient features of the program to participants and that although the provision of information on child-centered issues and accessing community-resources is important, it is not the most highly valued aspect of the home visitations. Therefore, the findings from the focus groups and FamPRS provide evidence for strength-based, family-centered practices being the mode of service delivery currently operationalized for Parenting Plus.

The underpinnings of strength-based, family-centered practices are found within the resource-based model of early intervention (Trivette et al., 1997). The resource model emphasizes both informal and formal supports and tends towards asset-driven, strengthbased modes of helping. While the community is the central feature of the resource-based model, it does not appear to have been fully developed in Parenting Plus. Even though Parenting Plus offers a strength-based approach to service delivery and is supposed to be characterized by program components that include "informal community support for all families and mechanisms for the co-ordination of services and supports" (Pipestone Health District, 1998 , p. 1), it would be wrong to assume that the community is the central feature of the intervention. Rather, the findings from the present evaluation confirm that, although Parenting Plus does have elements of the resource-based approach and appears to be delivering family-centered practices, initially, not all components of the model were developed to the same degree. In this respect, greater emphasis during the 
implementation phase of the program was directed towards developing sources of support and building on family strengths, while the other two components of the resource-based model, community resource mapping and building community capacity, were not fully developed. This in part occurred because of difficulties owing to partnership building and communication and the lack of knowledge and subsequent insufficient program resources directed towards this component of the model. Nevertheless, Parenting Plus continued to evolve and work towards assisting families to access intersectoral resources through the development of informal and formal community supports for the child and family being served.

Finally, it was found in the provision of strength-based family-centered practices that service needs to be rooted in the context of the family, community, and cultural codes in which it is being delivered. The findings from the interviews disclosed that a family's experiences with other types of service delivery, in particular child protection, could taint its current perception of early intervention services. This is even more significant to First Nation communities where already difficult intersectoral relationships with federal and provincial governments are exacerbated by historical prejudices. During the focus group sessions, parents discussed how having a FSW from the community helped engage and support families. This was especially important to First Nation focus group participants. Thus, it was a conscious decision on the part of Parenting Plus to hire FSWs from the communities in the health district so as to ensure that a strength-based approach to family-centered practices be delivered and operationalized. As both interview and focus group participants demonstrated, the extent to which families develop trust and accept program delivery is an important consideration that needs to be addressed in the broader 
context of home visitations and the models of service delivery they represent.

\section{Research Implications}

Findings from the present study clearly indicate that challenges existed in the implementation of Parenting Plus as modeled after Hawaii Healthy Start. Although other early intervention programs that follow a home-visitation model also report challenges in implementing the model prescribed, it is hard to compare models in terms of impact because of the different target audiences and objectives outlined for the various programs. Nevertheless, targeted interventions that feature paraprofessional home visitations continue to be widely endorsed because of the potential untested advantages they offer. The present study proposes the following as an aid in understanding the benefits of such a mode of service delivery.

\section{Targeted Early Interventions}

Guterman (1999) states three possible explanations as to why a trend favoring population-based as opposed to targeted approaches might exist:

1. Psychosocial screens hold questionable predictive accuracy in identifying future maltreatment, and therefore screen in families with low propensity to maltreat and for which services will not leverage further risk reduction.

2. Psychosocial screens employed in studies, in their effort to screen for "high risk", may screen in higher proportions of families less amenable to change, and simultaneously screen out more families amenable to change.

3. Psychosocial screens may serve to identify and enroll higher proportions of families for which home visitations are not appropriately matched to family need. (p. 875).

Thus, in order to make a rationale choice among early intervention strategies (for example clinical, universal, or targeted approaches), certain data requirements must be 
addressed. Parenting Plus is no exception in this regard. As a targeted intervention, a number of questions arise as to the efficacy of the intervention being practiced. Since the targeted approach to early intervention involves the use of screens, both the positive predictive value and the negative predictive value of the screens needs to be understood. What is known about the risk factors and the potency of the fixed markers on the screen to accurately identify families who would benefit from home visitations and are amenable to change needs clarification. What is known about the casual risk factors and which factors singularly or in combination can be changed and alter the risk status of the individual or family being served needs also to be investigated. In this respect, recent studies have reported that the presence of domestic violence limits the effectiveness of home visitations to prevent child maltreatment (Eckenrode et al., 2000). Consequently, the research implication for future evaluative efforts of early interventions like Parenting Plus that take a targeted approach is that they should examine the effect that a range of poor parenting practices can have on the health and development of the child and the family.

\section{Paraprofessional Home Visitations}

It is often assumed that in providing paraprofessional home visitations from a member of the community being served that a "strong focus on cultural sensitivity may lead to better success in engaging hard to reach families" (Duggan, Windham et al., 2000, p.257). Although the present study supports the contention that having a paraprofessional from the community is a desirable feature from the participants' perspective, further research needs to explore the extent to which paraprofessionals both engage and retain 
families in comparison to other forms of service delivery. Future research needs to not only look at the mode of service delivery but, more importantly, at how the characteristics of the home visitor can effect change in the participant and what level of experience or education in the paraprofessional is best suited to a particular client population. Other issues relating to home visitations pertain to the role of professionals in the community and when the paraprofessional should involve professionals. Should a client's risk level be used as an indicator for when the paraprofessional should team with other professionals, or are their other fixed markers or indicators that would prove to be more accurate predictors of when partnerships should be established so as to provide a continuum of services?

\section{Implementation Evaluation}

Increasingly stakeholders and policy decision makers are calling for evaluations that seek to explain the processes involved in newly formed organizations or established programs. These assessments are known as implementation evaluations and are becoming popularized as a means to connect what actually happens in a program to its intended outcomes (Patton, 1997a). For pilot projects, implementation evaluations are important because they can inform subsequent efforts and stabilize the program. Moreover, they allow for the opportunity to ascertain a project's viability. This becomes increasingly important when evaluators are attempting to discern the relevance and power of outcomes. Stakeholders are better able to determine the causal linkages between project planning, development, service delivery, and outcomes that either facilitated or impeded program implementation. Insight into the variables that either facilitate or impede a 
program's implementation are as critical as the establishment of partnerships for the delivery of intersectoral programs.

For programs modeled on Hawaii Healthy Start, Duggan, McFarlane et al.(1999) suggest that an examination of process variables relating to home visitations needs to be considered from the perspectives of the providers and the families. Moreover, the local values and needs of a district should be considered in relation to how they effect program implementation. Thus it would be inappropriate to transfer a home visiting program to another district simply because it had success in one setting without considering the new context (Duggan, Windham et al., 2000). As early intervention efforts continue to develop, provincially and nationally, implementation evaluations need to be undertaken during the formative phase of a program. Documenting the fidelity of early intervention efforts during implementation will provide information for reflection on how adaptations and modifications to a program have effected the system of service delivery and how these short-cycle decisions will effect its sustainability.

\section{Short-term Outcome Evaluation}

Early intervention efforts that have been successfully piloted, such that program stabilization has occurred, should focus on determining an intervention's effectiveness. For Parenting Plus, within a second generation research context (Guralnick, 1997), the program needs to carefully delineate what child and family characteristics are amenable to change and what program features are more likely to contribute to this change. Moreover, in strength-based models like Parenting Plus, outcome measurement is further complicated because the intervention is broad based, encompasses a variety of domains, 
and incorporates resources both informally and formally (Powell et al., 1997). Subsequently, innovative methodologies and instrumentation are required to determine not only changes in child health and development but family functioning, as well. In terms of outcome research, it is clear that control groups are advantageous. However, not all early intervention home visitations have the resources for large-scale randomized trials (Duggan, Windham et al., 2000). Consequently, Duggan, McFarlane et al. (1999) recommend that policy makers and planners incorporate the strongest internal evaluation methodologies that have been subjected to academic scrutiny when planning for the implementation and delivery of early intervention services.

\section{Longitudinal Research}

The benefits of early intervention are not just limited to the short-term health and well-being of children and parents. Long-term societal benefits are also reported in the research literature that link fewer demands being placed on social welfare, education, health, and justice services to an improved parental and child life course. Added social benefits found in the long-term research report increased rates of high school completion, increased employment, and decreased reliance on social assistance (McCormick, 1989; McCormick, Brooks-Gunn, Workman-Daniels, Turner, \& Peckham, 1992). Moreover, research into the economic benefits indicate that for every dollar spent on early childhood intervention, six to seven dollars are saved in later remedial education, social services, justice services, and health care (Barnett, 1993; Barnett \& Escobar, 1990; Olds \& Kitzman, 1993).

Two implications regarding future research can be drawn. First, early intervention 
research needs to be conducted not only to validate long-term benefits but also to report on the maintenance of home-visitation effects once the visits are discontinued. Second, future research needs to explore the variables, factors, and mechanisms that place children at-risk, as well as the roots of resilience that allow them to overcome adversity (Werner \& Smith, 1992). According to Rutter (1989), epidemiological studies that include a longitudinal component are receiving increased recognition because of the importance of understanding why some individuals exposed to risk factors do not develop psychosocial disorders. Unfortunately, much of the research has focused on adolescents and children in their middle years to the neglect of early childhood and longitudinal studies. Research which incorporates both quantitative and qualitative methodologies and monitors high-risk populations over time to determine risk and protective factors, including positive attributes and indicators of successful adaptation, is both desirable and necessary if social-policy planning, interventions, and programing are to be effective.

\section{Conclusion}

The present study is distinctive in two important aspects. As an implementation evaluation, it is unique in both its evaluative framework and the methods used to answer the research questions. The study sought to explore the implementation process for Parenting Plus, an early childhood development home-visitation program modeled after Hawaii Healthy Start. As Parenting Plus was in its developmental stages, it was not thought that an evaluation examining the outcomes of the program was warranted at this time. The rationale for this was twofold. First, because Parenting Plus is a pilot program and at the time of evaluation had been in operation for just over a year, any changes in 
indicators of outcome effects might not be wholly attributable to intervention efforts. Thus, because the program had not stabilized into a standardized form of service delivery, an evaluation effort that sought to explore outcome effects would be flawed. Second, during initial discussions regarding the evaluation framework with Parenting Plus program staff and stakeholders, it was disclosed that there had been significant difficulties in establishing a systematic screening process. As a result, program numbers were being compromised, weakening a study that examined quantitative indicators of positive treatment effects. In adopting an implementation evaluation Patton's (1997a) utilization-focused evaluation proved a valuable tool to augment efforts. Within this context both qualitative and quantitative methods are used to collect data, report results, and synthesize findings.

In implementing the program protocol, hospital-based screening for all live births in the Pipestone Health District proved unworkable. Nurses involved in maternity found that, due to the birth mother's relatively brief stay in the hospital, the collection of information pertaining to the completion of the medical record screen was not feasible. Moreover, stakeholders raised ethical considerations in regard to the relevancy of the screen and its ability to predict future psychosocial adjustment. Clearly, if targeted interventions are going to be successful it is important that both the stakeholders and the public be involved in the development and implementation of the program. Public seminars that provide a forum for input and education about a program need to be planned for when implementing early intervention programs that rely on informal and community support. Moreover, the greater the community awareness of a program the better the chance that participants will enroll because of knowledge of the benefits the 
program can provide. Thus, their involvement is believed to contribute to reduced attrition rates and ultimately higher enrollment numbers.

Although initial difficulties in obtaining screens resulted in the program operating at under capacity, recent changes have proved positive and benefited the growth and development of the program. Renewed partnership building between Parenting Plus and other health districts, hospitals, First Nations, family doctors, and public- and community-health nurses has eliminated some of the barriers to implementation that Parenting Plus initially experienced. Consequently, a more systematic screening process is in place, resulting in a higher number of positive screens and, eventually, a larger number of parents participating in the program. This, in turn, has resulted in Parenting Plus operating at near capacity for its first year of operation. However, because of a more systematic screening process, increased responsibilities and demands are being placed on the program co-ordinator that were not anticipated during program implementation.

The program implemented and now in operation is strength-based and maps very closely with the components of the Hawaii Healthy Start model. Providing supportive services in parent skill building, emotional support, information on child care, and linkages to intersectoral resources are the salient features of both the Hawaii Healthy Start model and Parenting Plus. This type of service delivery meets or exceeds parents expectations and is viewed positively by participants parents. Although, there was a difference of opinion among stakeholders involved in health-care delivery as to what type of service-paraprofessional or professional-would better benefit families, having local community paraprofessionals has aided in establishing trust and rapport with participating parents. 
Public-health nurses, community-health nurses, doctors, and hospitals are integral components in the referral process and are important to program promotion and implementation within the district. An advisory committee has been established, an initiative that has been warmly received by stakeholders. Thus, policy and program recommendations speak to the need for intersectoral involvement as being crucial to a strength-based pilot project's success. In addition, more time needs to be allocated, prior to program implementation for partnership building with stakeholders and the public. In this respect, public education and awareness surrounding the program model's feasibility and applicability would aid in alleviating misconceptions and misunderstandings, build partnerships, and facilitate program implementation among stakeholders.

In conclusion, general findings in the research literature agree that home visitations, either alone or in combination with a center-based approach, have demonstrated consistent results in improving maternal and child health, providing social support, and reducing child abuse and neglect. Furthermore, there seems to be consensus that, in the short and long term home visitations are economically viable and potentially provide positive economic benefits. Thus, as the social environment is changing dramatically resulting in increasing numbers of children at-risk, it is vital that early intervention efforts, like Parenting Plus, continue to be implemented and generalized within an integrated system of service delivery. 


\section{REFERENCES}

Backett, K. C., \& Davison, C. (1995). Life course and lifestyle: the social and cultural location of health behaviors. Social Science and Medicine, 40(5), 629-638.

Barnett, S. W. (1993). Economic evaluation of home visiting programs. The Future of Children. 3 (3), 93-113.

Barnett, S. W. (1997). The effects of early intervention on maltreating parents and their children. In M. J. Guralnick (Ed.), The effectiveness of early intervention. (pp. 147170). Baltimore, MD: Paul H. Brookes Publishing Co.

Barnett, S. W., \& Escobar, C. M. (1990). Economic costs and benefits of early intervention. In S. J. Meisels \& J. P. Shonkoff (Eds.), Handbook of early childhood intervention (pp. 560-582). New York, NY: Cambridge University Press.

Blair, C., \& Ramey, C. T. (1997). Early intervention for low-birth-weight infants and the path to second-generation research. In M. J. Guralnick (Ed.), The effectiveness of early intervention. (pp. 77-98). Baltimore, MD: Paul H. Brookes Publishing Co.

Britt, D. W. (1998). Reaching out and making a difference: The context of meaning in a home based preschool program. Journal of Community Psychology, 26(2), 103-118.

Bronfenbrenner, U. (1979). The ecology of human development: Experiments by nature and design. Cambridge, MA: Harvard University Press.

Campbell, E., \& Jones, G. (2002). Sources of stress experienced by elite male wheelchair basketball players. Adapted Physical Activity Quarterly, 19(1), 82-99.

Conrad, K. R., Randolph, F. L., Kirby, M., \& Bebout, R. R. (1999). Creating and using logic models: Four perspectives. In J. Keldon, \& J. Conrad (Eds.), Homelessness prevention in treatment of substance abuse and mental illness: Logic models and implementation of eight American projects (pp. 17-31). Chicago, IL: The Haworth Press.

Creswell, J. W. (1998). Qualitative inquiry and research design: Choosing among five traditions. Thousand Oaks, CA: Sage.

Duggan, A., McFarlane, E., Windham, A., Rohde, C., Salkever, D., Fuddy, L., Rosenberg, L. A., Buchbinder, S. B., \& Sia, C. J. (1999). Evaluation of Hawaii's Healthy Start program. The Future of Children 9(1), 66-90.

Duggan, A., Windham, A., McFarlane, E., Fuddy, L., Rohde, C., Buchbinder, S., \& Sia, C. (2000). Hawaii's Healthy Start Program for home visiting for at-risk families: Evaluation of family identification, family engagement, and service delivery. Pediatrics, 105 (1), 250-259. 
Dunst, C. J., \& Trivette, C. M. (1997). Early intervention with young at-risk children and their families. In R. Ammerman \& M. Hersen (Eds.), Handbook of prevention and treatment with children and adolescents: Intervention in the real world (pp. 157-80). New York, NY: Wiley and Sons.

Dyson, B., \& O'Sullivan, M. (1998). Innovation in two alternative elementary school programs: why it works. Research Quarterly for Exercise and Sport, 69(3), 242-253.

Eckenrode, J., Ganzel, B., Henderson, C. R., Smith, E., Olds, D. L., Powers, J., Cole, R., Kitzman, H., \& Sidora, K. (2000). Preventing child abuse and neglect with a program of nurse home visitation: the limiting effects of domestic violence. Journal of the American Medical Association, 284(11), 1385-1391.

Fetterman, D. M. (1993). Speaking the language of power: Communication, collaboration, and advocacy. London, ENG: Falmer Press.

Fetterman, D. M. (1994). Empowerment evaluation. Evaluation Practice, 15, 1-15.

Fetterman, D. M. (1995). Empowerment evaluation. Thousand Oaks, CA: Sage.

Fetterman, D. M. (1996). Empowerment evaluation: An introduction to theory and practice. In D. M. Fetterman, S. J. Kaftarian, \& A. Wandersman (Eds.) Empowerment evaluation: Knowledge and tools for self-assessment and accountability (pp. 3-48). Thousand Oaks, CA: Sage.

Fetterman, D. M. (1997). Empowerment evaluation and higher education. In E. Chelimsky \& W. R. Shadish (Eds.), Evaluation for the 21st century: A handbook (pp. 381-395). Thousand Oaks, CA: Sage.

Fetterman, D. M. (1999). Reflections on empowerment evaluation: Learning from experience. The Canadian Journal of Program Evaluation, Special Issue, 5-37.

Fishman, D. B. (1997). Postmodernism comes to program evaluation III [Review of the book Action Research]. Evaluation and program planning, 20(3), 301-310.

Fontana, A., \& Frey, J. H. (1994). Interviewing: The art of science. In N. K. Denzin \& Y. S. Lincoln (Eds.), Handbook of qualitative research (pp. 105-117). Thousand Oaks, CA: Sage.

Frailberg, S. (1980). Clinical studies in infant mental health: The first year of life. New York, NY: Basic Books.

Government of Saskatchewan. (1993). Children first: An invitation to work together: Creating Saskatchewan's action plan for children. Regina, SK: Author. 
Government of Saskatchewan. (2001). SchoolPlus a vision for children and youth: Toward a new school, community and human partnership in Saskatchewan. Regina, SK: Authour.

Gray, J. D., Cutler, C. A., Dean, J. G., \& Kempe, C. H. (1979). Prediction and prevention of child abuse and neglect. Journal of Social Issues, 35, 127-139.

Gray, J. D., \& Halpren, R. (1988). Early parenting intervention to prevent child abuse: $A$ meta-analysis: Final report to the National Center on Child Abuse and Neglect. Washington, D. C.: U. S. Department of Health and Human Services.

Greene, J. C. (1994). Qualitative program evaluation: Practice and promise. In N. K. Denzin \& Y. S. Lincoln (Eds.), Handbook of qualitative research (pp.105-117). Thousand Oaks, CA: Sage.

Guralnick, M. J. (1997). Second generation research in the field of early intervention. In M. J. Guralnick (Ed.), The Effectiveness of Early Intervention. (pp. 3-22). Baltimore, MD: Paul H. Brookes Publishing Co.

Guralnick, M. J. (1998). Effectiveness of early intervention for vulnerable children: A developmental perspective, American Journal on Mental Retardation, 102(4), 319345 .

Guteng, S. I., \& Chappell, B. (2000). Developmental practicum experiences of preservice teachers in deaf education: implications for-practicum placement and faculty-student collaborative research. American Annals of the Deaf, 145(5), 411-419.

Guterman, N. B. (1999). Enrollment strategies in early home visitation to prevent physical child abuse and neglect and the "universal versus targeted" debate: A metaanalysis of population-based and screening-based programs. Child Abuse and Neglect, 23 (9), 863-890.

Hammond, H. (1999). Identifying best family-centered practices in early-intervention programs. Teaching Exceptional Children, 31(6), 42-46.

Hauser-Cram, P. (1990). Designing meaningful evaluations of early intervention services. In S. J. Meisels \& J. P. Shonkoff (Eds.), Handbook of early childhood intervention (pp. 583-601). New York, NY: Cambridge University Press.

Hawaii Department of Health, Maternal, and Child Health Branch (1992). Healthy Start: Hawaii's system of support services, 1992. Honolulu, Hawaii: Author.

Hawaii Department of Health, Maternal and Child Health Branch (1994a). Healthy Start: Hawaii's system of support services, 1991-1993. Honolulu, Hawaii: Author. 
Hawaii Department of Health, Maternal and Child Health Branch (1994b). Healthy Start Training Manual. Honolulu, Hawaii: Hawaii Family Stress Center.

Health and Welfare Canada. (1992). Brighter futures: Canada's action plan for children. Ottawa, ON: Author.

Hiatt, S. W., Sampson, D., \& Baird, D. (1997). Paraprofessional home visitation: Conceptual and pragmatic considerations. Journal of Community Psychology 25(1), 77-93.

Julian, D. A. (1997). The utilization of the logic model as a system level planning and evaluation device. Evaluation and Program Planning, 20(3), 251-257.

Julian, D. A., Jones, A., \& Deyo, D. (1995). Open systems evaluation and the logic model: Program planning and evaluation tools. Evaluation and Program Planning, 18(4), 333-341.

Keating, D. P., \& Mustard, J. F. (1993). Social economic factors and human development. In D. Ross (Ed.). Family security in insecure times (Vol. 1, pp. 87-105). Ottawa, ONT: National Forum on Family Security.

Kempe, H. (1976). Child abuse and neglect: The family and the community. Cambridge, MA: Ballinger Publishing Company.

Kitzman, H. J., Olds, D., Cole, R., \& Yoos, H. (1997). Challenges experienced by home visitors: A qualitative study of program implementation. Journal of Community Psychology, 25 (1), 95-109.

Kitzman, H. J., Yoos, H. L., Cole, R., Korfmacher, J., \& Hanks, C. (1997). Prenatal and early childhood home-visitation program processes: A case illustration. Journal of Community Psychology, 25, (1), p. 27-45.

Kruger, R. A., Casey, M. A. (2000). Focus groups: A practical guide for applied research $\left(3^{\text {rd }}\right.$ ed.). Thousand Oaks, CA: Sage.

Kvale, S. (1996). InterViews: An introduction to qualitative research interviewing. Thousand Oaks, CA: Sage.

Lee, I. (1993). A validation study of the Family-Centered Program Rating Scale. Unpublished doctoral dissertation, University of Kansas, Lawrence.

Le-Mare, L., \& Sohbat, E. (2002). Canadian students' perceptions of teacher characteristics that support or inhibit help seeking. Elementary School Journal, 102(3), 239-253.

McCormick, M. C. (1989). Long term follow up of infants discharged from neonatal intensive care units. Journal of the American Medical Association, 261, 1767-1772. 
McCormick, M. C., Brooks-Gunn, J., Workman-Daniels, K., Turner, J., \& Peckham, G. (1992). The health and developmental status of very low birth weight children at school age. Journal of the American Medical Association, 267, 2204-2208.

Miles, B. M., \& Huberman, M. A. (1994). Qualitative data analysis $\left(2^{\text {nd }}\right.$ ed). Beverly Hills, CA: Sage.

Murphy, D., \& Lee. I. M. (1992). Family-Centered Program Rating Scale-Users manual. Lawrence, KS: University of Kansas, Beach Center on Families and Disabilities.

Murphy, D., Lee, I. M., Turbiville, V. P., Turnbull, A. P., \& Summers, J. A. (1991). Family-Centered Program Rating Scale-Parents' scale. Lawrence, KS: University of Kansas, Beach Center on Families and Disabilities.

Murphy, D., Lee, I. M., Turnbull, A. M., \& Turbiville, V. P. (1995). The FamilyCentered Program Rating Scale: An instrument for program evaluation and change. Journal of Early Intervention, 19(1), 24-42.

National Center on Child Abuse Prevention Research (1996). Intensive Home visitation: A randomized trial, follow-up and risk assessment study of Hawaii's Healthy Start program. Washington, D. C.: U. S. Department of Health and Human Services.

Offord, D. R., Kraemer, H. C., Kazdin, A. E., Jensen, P. R., Harrington, R., \& Gardner, J. S. (1999). Lowering the burden of suffering: Monitoring the benefits of clinical, targeted, and universal approaches. In D. P. Keating \& C. Hertzman (Eds.), Developmental health and the wealth of nations: Social, Biological, and educational dynamics (pp. 293-310). New York, NY: Guilford.

Olds, D. L. (1987). Study of nurse home visitation for mothers and children. Washington, DC: National Centre for Nursing Research.

Olds, D. L. (1992). Home visitation for pregnant women and parents of young children. American Journal of Diseases of Children, 146, 704-708.

Olds, D. L., Henderson, C., Chamberlain, R, \& Tatelbaum, R. (1986). Preventing child abuse and neglect: A randomized trial of nurse home visitation. Pediatrics, 78, 65-78.

Olds, D. L., Henderson, C., Tatelbaum, R., \& Chamberlain, R. (1986). Improving the delivery of prenatal care and outcomes of pregnancy: A randomized trial of nurse home visitation. Pediatrics, 77, 16-28.

Olds, D. L., Henderson, C., Tatelbaum, R., \& Chamberlain, R. (1988). Improving the life course development of socially disadvantaged mothers: A randomized trial of nurse home visitation. American Journal of Public Health, 78, 1436-45. 
Olds, D. L., \& Kitzman, H. (1993). Review of research on home visiting for pregnant women and parents of young children. The Future of Children, 3(3), 53-90.

Patton, M. Q. (1990). Qualitative evaluation and research methods ( $2^{\text {nd }}$ ed.) Beverly Hills, CA: Sage.

Patton, M. Q. (1986). Utilization-focused evaluation ( $2^{\text {nd }}$ ed.). Beverly Hills, CA: Sage.

Patton, M. Q. (1997a). Utilization-focused evaluation: The new century text. Thousand Oaks, CA: Sage.

Patton, M. Q. (1997b). Toward distinguishing empowerment evaluation and placing it in a larger context. Evaluation Practice 18(2), 147-163.

Payne, S. (1999). Interview in qualitative research. In A. Memon \& R. Bull (Eds.), Handbook of the psychology of interviewing (pp. 89-102). Toronto, ONT: Wiley and Sons.

Pipestone Health District (1998). Proposal for Health Transition Funding: Pipestone Health District. Grenfall, SK: Author.

Pipestone Health District (2001). Parenting Plus the early childhood development program of the Pipestone Health District: Final results and evaluation report. Grenfall, SK: Author.

Pope, C., Ziebland, S., \& Mays, N. (2000). Qualitative research in health care: Analysing qualitative data. British Medical Journal, 320, 114-116.

Powell, D. S., Batsche, C. B., Ferro, J., Fox, L., \& Dunlap, G. (1997). A strength-based approach in support of multi-risk families: Principles and issues. Topics in Early Childhood Special Education, 17(1), 1-26.

Ramey, C. T. \& Ramey, S. L. (1993). Home visiting programs and the health and development of young children. The Future of Children, 3 (3), 129-139.

Rutter, M. (1989). Isle of Wright revisited: twenty-five years of child psychiatric epidemiology. Journal of Child Psychology and Psychiatry, 8, 1-11.

Sameroff, A. J. (1983). Developmental systems: Contexts and evolution. In W. Kessen (Ed.), History, theories, and methods. In P. H. Mussen (Ed.), Handbook of child psychology (Vol. 1, pp. 238-94). New York, NY: Wiley.

Sameroff, A. J. (1993). Models of development and developmental risk. In C. H. Zeanah (Ed.), Handbook of infant mental health (pp. 3-13). New York, NY: Guilford. 
Sameroff, A. J., \& Chandler, M. J. (1975). Reproductive risk and the continuum of caretaking causality. In F. D. Horowitz, M. Hetherington, S. Scarr-Salapatek, \& G. Siegel (Eds.), Review of child development research (pp. 187-244). Chicago: University of Chicago Press.

Sameroff, A. J., \& Fiese, B. H. (1990). Transactional regulation and early intervention. In S. Meisels \& J. Shonokoff (Eds.), Handbook of early childhood intervention. (pp. 119-149). Cambridge: Cambridge University Press.

Sameroff, A. J., \& Fiese, B. H. (2000a). Transactional regulation: The developmental ecology of early intervention. In S. Meisels \& J. Shonokoff (Eds.), Handbook of early childhood intervention ( $2^{\text {nd }}$ ed.) (pp. 135-159). Cambridge: Cambridge University Press.

Sameroff, A. J., \& Fiese, B. H. (2000b) Models of development and developmental risk. In C. H. Zeanah (Ed.), Handbook of infant mental health (pp. 3-19). New York, NY: Guilford.

Saskatchewan Health (1999). Priority population policy. Regina, SK: Author.

Saskatchewan Health (2000). Saskatchewan health information source book 2000. Regina, SK: Author.

Sechrest, L. E. (1997). [Review of the book Empowerment evaluation: Knowledge and tools for self-assessment and accountability]. Environment and Behavior, 29(3), 422426.

Shepardson, D. P. (1996). Social interactions and the mediation of science learning in two small groups of first-graders. Journal of Research in Science Teaching, 33(2), 159-178.

Shuy, R. W. (2002). In-person versus telephone interviewing. In J. F. Gubrium \& J. H. Holstein (Eds.), Handbook of interview research: Context and method (pp. 537-556). Thousand Oaks, CA: Sage.

Socolar, R. R., Fredrickson, D. D., Block, R., Moore, J. K., Tropez-Sims, S., \& Whitworth, J. M. (2001). State programs for medical diagnosis of child abuse and neglect: Case studies of five established or fledgling programs. Child Abuse and Neglect, 25(4), 441-455.

Stufflebeam, D. L. (1971). The relevance of the CIPP evaluation model for educational accountability. Paper presented at the annual meeting of the American Association of School Administrators, Atlantic City, NJ.

Stufflebeam, D. L. (1972). The relevance of the CIPP evaluation model for educational accountability. School Research Information Quarterly, 5(1), 3-6. 
Tashakkori, A., \& Teddlie, C. (1998). Mixed methodology: Combining qualitative and quantitative approaches. Thousand Oaks, CA: Sage.

Thomas, D. R. (2000). Qualitative data analysis: Using a general inductive approach. Retrieved April 20, 2002, from the University of Auckland, Health Research Methods Advisory Service Web site: http://www.auckland.ac.nz/ mch/hrmas.html.

Trivette, C. M., \& Dunst, C. J. (2000). Recommended practices in family-based practices. In S. Sandall, M. E. McLean, \& B. J. Smith (Eds.), DEC recommended practices in early intervention/early childhood special education (pp. 39-44). Longmont, CO: Sopris West.

Trivette, C. M., \& Dunst, C. J., \& Deal, A. G. (1997). Resource-based approach to early intervention. In S. K. Thurman, J. R. Cornwell, \& S. R. Gottwald (Eds.), Contexts of early interventions: Systems and settings (pp. 73-92). Baltimore, MD: Paul H. Brookes.

Trocme, N., \& Wolfe, D. (2001) Child maltreatment in Canada: Selected results from the Canadian incidence study of reported child abuse and neglect. Ottawa, ONT: Minister of Public Works and Government Services, Canada.

Wallach, V. A., \& Lister, L. (1995). Stages in the delivery of home-based services to parents at risk of child abuse: A Healthy Start experience. Scholarly Inquiry for Nursing Practice: An International Journal, 9(2), p. 159-173.

Wengraf, T. (2001). Qualitative research interviewing: Biographic, narrative, and semistructured methods. Thousands Oaks, CA: Sage.

Werner, E., \& Smith, R. (1992). Overcoming the odds: High risk children from birth to adulthood. Ithaca, NY: Cornell University Press.

Wolery, M. (2000). Behavioral and educational approaches to early intervention. In S. Meisels \& J. Shonokoff (Eds.), Handbook of early childhood intervention $\left(2^{\text {nd }}\right.$ ed.) (pp. 179-203). Cambridge: Cambridge University Press.

Wolfe, D. A. (1991). Preventing physical and emotional abuse of children. New York, NY: Guilford Press. 
Appendix 1 


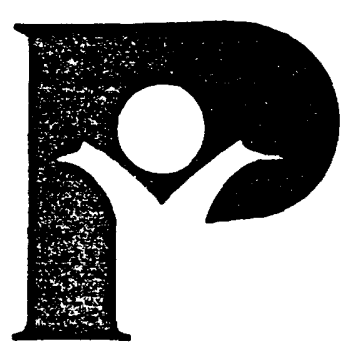

\title{
News \\ Release
}

\section{FOR RELEASE: \\ OCTOBER 19, 1999}

\section{Pipestone Health District Announces New Partnership with SaskTel}

ipestone is implementing an Early Childhood Development Program which consists of a omprehensive system of services and supports for families with children 0-5 years of age. ipestone Health District is pleased to announce its new partnership with SaskTel.

\author{
News Conference \\ 10:00 a.m., Tuesday, October 19, 1999 \\ SaskTel \\ $2^{\text {nd }}$ Floor \\ 2121 Saskatchewan Drive \\ Regina, SK
}

ariy childhood is more important in the development of happy, healthy children than was ice thought. Experiences that young children encounter during their first days, months, id years have a direct impact on how the brain is developed. Research now shows that the itical time factor in brain development is the first three years of life, and the most aportant year is the first one. Children who don't play much, or are rarely touched, often ) not develop normally. Simple things like talking to a baby, providing loving care, and ving a baby freedom to explore, as safety permits, are more important than was once alized. 
Appendix 2 


\section{Empowerment Evaluation Focus Group Guide}

Establishing a Mission

1. What do you feel is the mission of the program?

(i) Why did you join the program and what are the expected results?

(ii) What are some key phrases that describe the mission statement of the program?

Taking Stock

2. What are the most significant features and/or activities associated with the program?

(i) What are the top five features or activities about this program that are important to you?

(ii) Can you rate each activity on a 1 to 10 scale with 10 being the highest and 1 being the lowest?

3. Can you describe or explain what your ratings mean?

(i) What are the positive basis for your ratings?

(ii) What are the negative basis for your ratings?

Planning for the Future

4. How would you like to improve on what you do well or not so well?

(i) What are your goals based on the activities listed when taking stock?

(ii) What strategies will enable you to attain your goals?

(iii)What resources do you require in order to assist you in attaining your goals?

(iv) What forms of documentation are required to monitor progress towards the specified goals?

(v) How is this documentation related to the specified goals? 
Appendix 3 


\title{
FAMILY-CENTERED PROGRAM RATING SCALE
}

\section{PARENT'S SCALE}

\author{
Douglas L. Murphy \\ Ilene M. Lee \\ Vicki Turbiville \\ Anne P. Turnbull \\ Jean Ann Summers
}

(C) Beach Center on Families and Disability The University of Kansas

Adapted with permission 


\section{Family-Centered Program Rating Scale \\ Parent's Scale}

We invite parents of the Parenting Plus program to participate in the evaluation of the program by completing the following rating scale. Your participation is voluntary and the evaluation is of minimal risk. There are lots of different ways programs can serve families and their young children. Which ways are important to you? How well do you think this program is doing? Your response to these questions will help us evaluate this program and plan improvements.

Directions: Each statement on this rating scale finishes a sentence, which begins with the words at the top of the section. For example, statements in the first section begin with:

\section{IN THIS PROGRAM...}

All of the statements in the first section finish this sentence. There are four sections; each section has a different beginning. Read each statement and mark it two times:

\begin{tabular}{|c|c|c|c|c|c|c|c|c|c|}
\hline \multicolumn{2}{|r|}{$\begin{array}{l}\text { Tell how well Parenting Plus is doing on each item. } \\
\text { Circle the letters that most closely tell us your } \\
\text { opinion about how Parenting Plus is doing. }\end{array}$} & \multicolumn{8}{|c|}{$\begin{array}{l}\text { Tell how important the item is to you, personally. } \\
\text { Circle the letters that most closely tell us how } \\
\text { important this item is to you. }\end{array}$} \\
\hline \multicolumn{2}{|r|}{$\mathbf{P}=$ Poor } & \multicolumn{8}{|c|}{$\mathrm{N}=$ Not Important } \\
\hline \multicolumn{2}{|r|}{ OK=Okay } & \multicolumn{8}{|c|}{ SI=Somewhat Important } \\
\hline \multicolumn{2}{|r|}{ G=Good } & \multicolumn{8}{|c|}{ I=Important } \\
\hline \multicolumn{2}{|r|}{$E=$ Excellent } & \multicolumn{8}{|c|}{ VI=Very Important } \\
\hline \multicolumn{10}{|c|}{$\begin{array}{l}\text { Start Here } \\
\text { A. In this program... }\end{array}$} \\
\hline 1. & $\begin{array}{l}\text { meetings with my family are scheduled when and } \\
\text { where they are most convenient for us. }\end{array}$ & $\mathbf{P}$ & OK & $\mathbf{G}$ & $\mathbf{E}$ & NI & SI & I & VI \\
\hline 2. & $\begin{array}{l}\text { the information staff members give my family } \\
\text { members helps us make decisions about our child. }\end{array}$ & $\mathbf{P}$ & $\mathbf{O K}$ & G & $\mathbf{E}$ & NI & SI & I & VI \\
\hline 3. & $\begin{array}{l}\text { Someone on the staff can help my family get } \\
\text { services from other agencies. }\end{array}$ & $\mathbf{P}$ & $\overline{\mathrm{OK}}$ & $\mathbf{G}$ & $\mathbf{E}$ & NI & SI & I & VI \\
\hline 4. & $\begin{array}{l}\text { Services can change quickly when my family's or } \\
\text { child's needs change. }\end{array}$ & $\mathbf{P}$ & OK & $\mathbf{G}$ & $\mathbf{E}$ & NI & SI & I & VI \\
\hline 5 . & $\begin{array}{l}\text { services are planned with my family's } \\
\text { transportation and scheduling needs in mind. }\end{array}$ & $\mathbf{P}$ & OK & $\mathbf{G}$ & $\mathbf{E}$ & NI & SI & $\mathbf{I}$ & VI \\
\hline
\end{tabular}




\begin{tabular}{|c|c|c|c|c|c|c|c|c|c|}
\hline 6. & $\begin{array}{l}\text { someone on the staff can help my family } \\
\text { communicate with all the professionals serving us } \\
\text { and our child. }\end{array}$ & $\mathbf{P}$ & $\mathbf{O K}$ & $\mathbf{G}$ & $\mathbf{E}$ & NI & SI & $\mathbf{I}$ & VI \\
\hline 7. & $\begin{array}{l}\text { the program administrator makes my family feel } \\
\text { comfortable when we have questions or complaints. }\end{array}$ & $\mathbf{P}$ & OK & $\mathbf{G}$ & $\mathbf{E}$ & NI & SI & $\mathbf{I}$ & VI \\
\hline 8. & goal setting is used as a "plan of action". & $\mathbf{P}$ & $\mathbf{O K}$ & $\mathbf{G}$ & $\mathbf{E}$ & NI & SI & $\mathbf{I}-$ & VI \\
\hline 9. & $\begin{array}{l}\text { there is a comfortable way to work out } \\
\text { disagreements between families and staff members. }\end{array}$ & $\mathbf{P}$ & OK & $\mathbf{G}$ & $\mathbf{E}$ & NI & SI & I & VI \\
\hline & & \multicolumn{4}{|c|}{$\begin{array}{c}\text { How well does your } \\
\text { program do this? } \\
P=\text { Poor } \\
O K=\text { Okay } \\
G=\text { Good } \\
E=\text { Excellent }\end{array}$} & \multicolumn{4}{|c|}{$\begin{array}{c}\text { How important is this } \\
\text { to you? } \\
\text { NI=Not Important } \\
\text { SI=Somewhat Important } \\
\text { I=Important } \\
\text { VI=Very Important }\end{array}$} \\
\hline
\end{tabular}

B. The program...

\begin{tabular}{|c|c|c|c|c|c|c|c|c|c|}
\hline 10 . & $\begin{array}{l}\text { helps my family when we want information about, } \\
\text { jobs, money, counseling, housing or other basic } \\
\text { family needs. }\end{array}$ & $\mathbf{P}$ & $\overline{\text { OK }}$ & $\mathbf{G}$ & $\mathbf{E}$ & NI & SI & I & VI \\
\hline 11. & $\begin{array}{l}\text { gives the other children in my family support and } \\
\text { information about their newborn brother or sister. }\end{array}$ & $\mathbf{P}$ & $\overline{\mathbf{O K}}$ & $\mathbf{G}$ & $\mathbf{E}$ & NI & $\overline{\text { SI }}$ & $\overline{\mathbf{I}}$ & VI \\
\hline 12. & $\begin{array}{l}\text { gives us information on how to meet other families } \\
\text { of children with similar needs. }\end{array}$ & $\mathbf{P}$ & OK & $\mathbf{G}$ & $\mathbf{E}$ & NI & SI & I & VI \\
\hline 13. & $\begin{array}{l}\text { offers special times for fathers to talk with other } \\
\text { fathers and with the staff. }\end{array}$ & $\mathbf{P}$ & OK & $\mathbf{G}$ & $\mathbf{E}$ & NI & SI & I & VI \\
\hline 14. & $\begin{array}{l}\text { offers information in a variety of ways (written, } \\
\text { videotape, cassette tape, workshop, etc.). }\end{array}$ & $\mathbf{P}$ & OK & $\mathbf{G}$ & $\mathbf{E}$ & NI & SI & I & VI \\
\hline 15 & $\begin{array}{l}\text { helps my family expect good things in the future } \\
\text { for ourselves and our children. }\end{array}$ & $\mathbf{P}$ & $\overline{\mathrm{OK}}$ & $\mathbf{G}$ & $\mathbf{E}$ & NI & SI & I & $\mathbf{V I}$ \\
\hline
\end{tabular}

\section{Staff members...}

\begin{tabular}{|c|c|c|c|c|c|c|c|c|c|}
\hline 16. & $\begin{array}{l}\text { are available to go to doctors or other service } \\
\text { providers with my family to help ask questions, } \\
\text { sort out information, and decide on services. }\end{array}$ & $\mathbf{P}$ & $\mathbf{O K}$ & $\overline{\mathbf{G}}$ & $\mathbf{E}$ & $\mathbf{N I}$ & SI & $\mathbf{I}$ & VI \\
\hline 17. & $\begin{array}{l}\text { help my family learn how to teach our child } \\
\text { different skills. }\end{array}$ & $\mathbf{P}$ & $\overline{\mathbf{O K}}$ & $\mathbf{G}$ & $\mathbf{E}$ & NI & SI & I & VI \\
\hline 18. & $\begin{array}{l}\text { give information to help my family explain our } \\
\text { needs to friends and other family members. }\end{array}$ & $\mathbf{P}$ & OK & $\mathbf{G}$ & $\mathbf{E}$ & NI & SI & I & VI \\
\hline 19. & help my family plan for the future. & $\mathbf{P}$ & $\overline{\mathbf{O K}}$ & $\bar{G}$ & $\bar{E}$ & NI & SI & I & $\mathbf{V I}$ \\
\hline 20. & $\begin{array}{l}\text { don't ask my family about personal matters unless } \\
\text { it is necessary. }\end{array}$ & $\mathbf{P}$ & $\mathbf{O K}$ & $\mathbf{G}$ & $\mathbf{E}$ & NI & SI & I & VI \\
\hline 21. & $\begin{array}{l}\text { respect whatever level of involvement my family } \\
\text { chooses in making decisions. }\end{array}$ & $\overline{\mathbf{P}}$ & $\overline{\mathbf{O K}}$ & $\mathbf{G}$ & $\mathbf{E}$ & NI & SI & $\mathbf{I}$ & $\mathbf{V I}$ \\
\hline 22. & don't rush my family to make changes. & $\mathbf{P}$ & OK & $\mathbf{G}$ & $\mathbf{E}$ & NI & SI & I & VI \\
\hline 23. & $\begin{array}{l}\text { help my family feel we can make a positive } \\
\text { difference in our child's life. }\end{array}$ & $\mathbf{P}$ & OK & $\overline{\mathbf{G}}$ & $\bar{E}$ & NI & SI & $\mathbf{I}$ & VI \\
\hline 24. & $\begin{array}{l}\text { give my family time to talk about our experiences } \\
\text { and things that are important to us. }\end{array}$ & $\mathbf{P}$ & $\overline{\mathbf{O K}}$ & $\bar{G}$ & $\mathbf{E}$ & NI & SI & $\bar{I}$ & VI \\
\hline 25. & are honest with my family. & $\mathbf{P}$ & $\overline{O K}$ & $\bar{G}$ & $\mathbf{E}$ & NI & SI & I & $\mathbf{V I}$ \\
\hline
\end{tabular}




\begin{tabular}{|c|l|l|l|l|l|}
\hline 26. & $\begin{array}{l}\text { create ways for my family to be involved in } \\
\text { making decisions about services. }\end{array}$ & OK & G & E \\
\hline 27. & $\begin{array}{l}\text { give my family clear and complete information } \\
\text { about our child. }\end{array}$ & P & OK & G & E \\
\hline 28. & $\begin{array}{l}\text { tell my family what they have learned about our } \\
\text { child and family. }\end{array}$ & P & OK & G & E \\
\hline 29. & $\begin{array}{l}\text { don't act rushed or in a hurry when they meet with } \\
\text { me or my family. }\end{array}$ & P & OK & G & E \\
\hline
\end{tabular}

How well does your program do this?

$\mathrm{P}=$ Poor

$\mathrm{OK}=$ Okay

$\mathrm{G}=\mathrm{Good}$

$E=$ Excellent

\begin{tabular}{|l|l|l|l|}
\hline NI & SI & I & VI \\
\hline NI & SI & I & VI \\
\hline NI & SI & I & VI \\
\hline NI & SI & I & VI \\
\hline
\end{tabular}

How important is this to you?

$\mathrm{NI}=$ Not Important

SI=Somewhat Important $I=$ Important

$\mathrm{VI}=$ Very Important

\section{Staff members...}

\begin{tabular}{|c|c|c|c|c|c|c|c|c|c|}
\hline 30. & $\begin{array}{l}\text { don't ask my family to repeat information that is } \\
\text { already on file. }\end{array}$ & $\mathbf{P}$ & OK & $\mathbf{G}$ & $\mathbf{E}$ & NI & SI & I & VI \\
\hline 31. & $\begin{array}{l}\text { don't try to tell our family what we need or don't } \\
\text { need. }\end{array}$ & $\mathbf{P}$ & OK & $\overline{\mathbf{G}}$ & $\bar{E}$ & NI & $\overline{\text { SI }}$ & I & VI \\
\hline 32. & $\begin{array}{l}\text { help my family feel more confident about working } \\
\text { with professionals. }\end{array}$ & $\mathbf{P}$ & OK & $\mathbf{G}$ & $\mathbf{E}$ & NI & SI & I & $\overline{V I}$ \\
\hline 33. & $\begin{array}{l}\text { give my family clear and complete information } \\
\text { about families rights. }\end{array}$ & $\mathbf{P}$ & OK & $\mathbf{G}$ & $\mathbf{E}$ & NI & SI & I & VI \\
\hline 34. & $\begin{array}{l}\text { give my family clear and complete information } \\
\text { about available services. }\end{array}$ & $\mathbf{P}$ & OK & $\mathbf{G}$ & $\mathbf{E}$ & NI & SI & I & VI \\
\hline 35. & $\begin{array}{l}\text { help my family feel more comfortable when } \\
\text { asking for help and support from friends and other } \\
\text { family members. }\end{array}$ & $\mathbf{P}$ & OK & $\mathbf{G}$ & $\overline{\mathbf{E}}$ & NI & $\overline{\text { SI }}$ & I & VI \\
\hline 36. & $\begin{array}{l}\text { regularly ask my family about how well the } \\
\text { program is doing and what changes we might like } \\
\text { to see. }\end{array}$ & $\mathbf{P}$ & OK & $\mathbf{G}$ & $\bar{E}$ & NI & $\overline{\text { SI }}$ & I & VI \\
\hline 37. & offer to visit my family in our home. & $\mathbf{P}$ & OK & $\mathbf{G}$ & $\mathbf{E}$ & NI & SI & I & $\mathbf{V I}$ \\
\hline 38. & $\begin{array}{l}\text { offer ideas on how my family can have fun with } \\
\text { our children. }\end{array}$ & $\mathbf{P}$ & OK & $\mathbf{G}$ & $\overline{\mathbf{E}}$ & NI & SI & I & VI \\
\hline 39. & $\begin{array}{l}\text { treat my family as the true experts on our child } \\
\text { when planning and providing services. }\end{array}$ & $\mathbf{P}$ & OK & $\mathbf{G}$ & $\mathbf{E}$ & NI & SI & $\mathbf{I}$ & VI \\
\hline 40. & $\begin{array}{l}\text { Give my family clear and complete explanations } \\
\text { about our child. }\end{array}$ & $\mathbf{P}$ & OK & $\mathbf{G}$ & $\mathbf{E}$ & NI & SI & I & VI \\
\hline 41. & $\begin{array}{l}\text { Help my family learn how we can help our } \\
\text { children feel good about themselves. }\end{array}$ & $\mathbf{P}$ & OK & $\mathbf{G}$ & $\mathbf{E}$ & NI & SI & I & VI \\
\hline 42. & Don't overwhelm us with too much information. & $\mathbf{P}$ & OK & $\mathbf{G}$ & $\mathbf{E}$ & NI & SI & $\mathbf{I}$ & VI \\
\hline 43. & $\begin{array}{l}\text { Get to know my family and let us get to know } \\
\text { them. }\end{array}$ & $\mathbf{P}$ & OK & $\mathbf{G}$ & $\mathbf{E}$ & NI & SI & $\mathbf{I}$ & $\overline{\mathbf{V I}}$ \\
\hline 44. & $\begin{array}{l}\text { Help my family use problem solving skills for } \\
\text { making decisions about ourselves and our } \\
\text { children. }\end{array}$ & $\mathbf{P}$ & OK & $\mathbf{G}$ & $\mathbf{E}$ & NI & SI & I & VI \\
\hline 45. & $\begin{array}{l}\text { Give information that helps my family with our } \\
\text { children's everyday needs (feeding, clothing, } \\
\text { playing, health care, safety, friendship, etc.) }\end{array}$ & $\mathbf{P}$ & OK & $\mathbf{G}$ & $\mathbf{E}$ & NI & SI & $\mathbf{I}$ & VI \\
\hline 46. & Help my family see what we are doing well. & $\mathbf{P}$ & OK & $\mathbf{G}$ & $\mathbf{E}$ & NI & SI & I & VI \\
\hline
\end{tabular}




\begin{tabular}{|c|l|l|l|l|l|}
\hline 47. & $\begin{array}{l}\text { Respect differences among children, families, and } \\
\text { families' ways of life. }\end{array}$ & $\mathbf{P}$ & $\mathbf{O K}$ & $\mathbf{G}$ & $\mathbf{E}$ \\
\hline 48. & $\begin{array}{l}\text { Ask my family's opinions and include us in the } \\
\text { process of understanding our child. }\end{array}$ & $\mathbf{P}$ & OK & $\mathbf{G}$ & $\mathbf{E}$ \\
\hline 49. & Are friendly and easy to talk to. & $\mathbf{P}$ & OK & $\mathbf{G}$ & $\mathbf{E}$ \\
\hline 50. & $\begin{array}{l}\text { Help my family feel more confident that we are } \\
\text { experts on our children. }\end{array}$ & $\mathbf{P}$ & OK & $\mathbf{G}$ & $\mathbf{E}$ \\
\hline 51. & Enjoy working with my family and child. & P & OK & $\mathbf{G}$ & $\mathbf{E}$ \\
\hline
\end{tabular}

How well does your program do this?

$\mathrm{P}=$ Poor

$\mathrm{OK}=$ Okay

$\mathrm{G}=\mathrm{Good}$

$E=$ Excellent

\begin{tabular}{|l|l|l|l|}
\hline NI & SI & I & VI \\
\hline NI & SI & I & VI \\
\hline NI & SI & I & VI \\
\hline NI & SI & I & VI \\
\hline NI & SI & I & VI \\
\hline
\end{tabular}

How important is this to you?

$\mathrm{NI}=$ Not Important

$\mathrm{SI}=$ Somewhat Important

I=Important

$\mathrm{VI}=$ Very Important

\section{Staff members...}

\begin{tabular}{|c|l|l|l|l|l|l|l|l|l|l|l|}
\hline 52. & Help my family to have a normal life. & $\mathbf{P}$ & $\mathbf{O K}$ & $\mathbf{G}$ & $\mathbf{E}$ & & NI & SI & I & VI \\
\hline 53. & $\begin{array}{l}\text { Explain how information about my family will be } \\
\text { used. }\end{array}$ & $\mathbf{P}$ & $\mathbf{O K}$ & $\mathbf{G}$ & $\mathbf{E}$ & & NI & SI & I & VI \\
\hline 54. & $\begin{array}{l}\text { Give my family information about how children } \\
\text { usually grow and develop. }\end{array}$ & $\mathbf{P}$ & $\mathbf{O K}$ & $\mathbf{G}$ & $\mathbf{E}$ & & NI & SI & I & VI \\
\hline 55. & $\begin{array}{l}\text { Help my family to see the good things we are } \\
\text { doing to meet our child's needs. }\end{array}$ & $\mathbf{P}$ & $\mathbf{O K}$ & $\mathbf{G}$ & $\mathbf{E}$ & & NI & SI & I & VI \\
\hline 56. & $\begin{array}{l}\text { Consider my family's strengths and needs when } \\
\text { planing to meet our child's needs. }\end{array}$ & $\mathbf{P}$ & $\mathbf{O K}$ & $\mathbf{G}$ & $\mathbf{E}$ & & NI & SI & I & VI \\
\hline
\end{tabular}

\section{My family...}

\begin{tabular}{|c|l|l|l|l|l|l|l|l|l|l|}
\hline 57. & Is included in all meetings about us and our child. & $\mathbf{P}$ & $\mathbf{O K}$ & $\mathbf{G}$ & $\mathbf{E}$ & & NI & SI & $\mathbf{I}$ & VI \\
\hline 58. & $\begin{array}{l}\text { Receives complete copies of all reports about us } \\
\text { and our child. }\end{array}$ & $\mathbf{P}$ & $\mathbf{O K}$ & $\mathbf{G}$ & $\mathbf{E}$ & & NI & SI & I & VI \\
\hline 59. & $\begin{array}{l}\text { Is an important part of the team when goal setting } \\
\text { is developed, reviewed, or changed. }\end{array}$ & $\mathbf{P}$ & $\mathbf{O K}$ & $\mathbf{G}$ & $\mathbf{E}$ & & NI & SI & I & VI \\
\hline
\end{tabular}

\section{E. Comments...}

What things about Parenting Plus make it especially helpful and welcoming?

What are the ways in which Parenting Plus could be more helpful and welcoming to your family? 
Appendix 4 


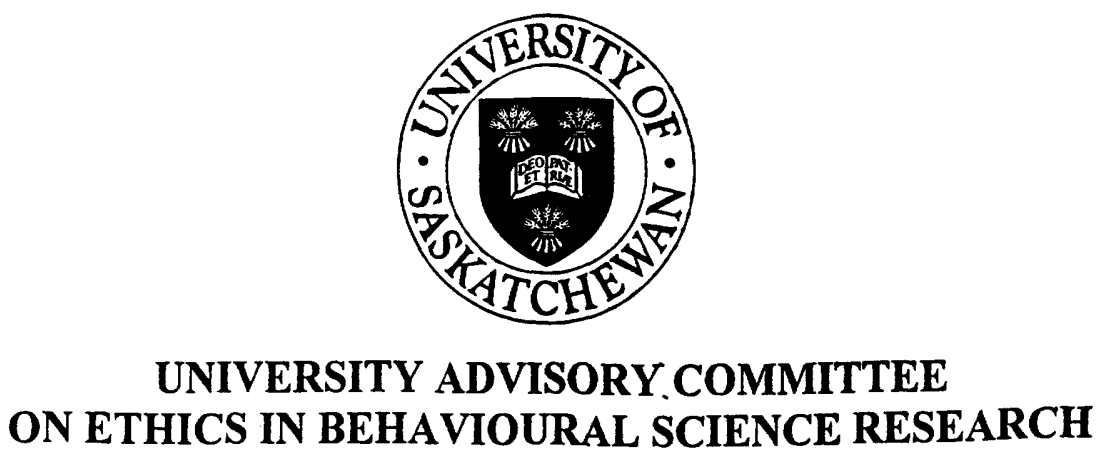

IME: V. Schwean (D. Mykota)

BSC\#: 2000-128

Educational Psychology and Special Education

ITE: August 31, 2000

e University Advisory Committee on Ethics in Behavioural Science Research has reviewed revisions to the Application for Ethics Approval for your study "The Implementation aluation of Parenting Plus, the Early Childhood Program of the Pipestone Health District" (003).

Your study has been APPROVED.

Any significant changes to your proposed study should be reported to the Chair for Committee consideration in advance of its implementation.

The term of this approval is for 5 years.

ish you a successful and informative study.

\section{5
er
ive
Et}

erie Thompson, Chair

iversity Advisory Committee

Ethics in Behavioural Science Research

'bjk 
Appendix 5 


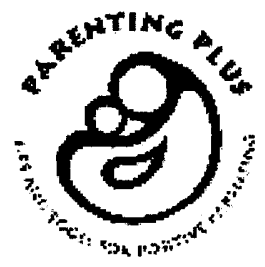

PARENTING PLUS

Pipestone Health District

\section{INTERVIEW CONSENT FORM AND STATEMENT OF CONFIDENTIALITY}

The Pipestone Health District is inviting stakeholders in the Early Childhood Development Program Parenting Plus home visitations to participate in the Early Childhood Development Program Parenting Plus evaluation. David Mykota, an Educational Psychology and Special Education Graduate student at the University of Saskatchewan is undertaking the evaluation. The title of the project is The Implementation Evaluation of Parenting Plus, the Early Childhood Development Program of the Pipestone Health District. The goal of this evaluation is to see how well the Parenting Plus program is doing, how well parent's home visiting programs are doing and how we could improve home visiting programs. Your role as a participant in the evaluation will be to participate in a minimum of one to a maximum of three interview sessions. The interview would be arranged at a centralized location and would take approximately two hours to complete each session for a total of two to six hours. The interview would allow you to participate in the evaluation of the Parenting Plus program.

The evaluation of the Parenting Plus program is of minimal risk. The purpose and possible benefits of the evaluation are to see:

- How this program makes a difference for the family and child

- How Pipestone's children and families are doing over time

- How well children's programs are working

- How we could improve the children's programs

- What other programs are needed

The evaluation is being conducted through the Pipestone Health District and the University of Saskatchewan and as a participant you have definite rights:

1. Your participation in any interviews is completely voluntary.

2. You are free to refuse to answer any question at any time and you are free to withdraw from the evaluation, at any time, without penalty.

3. As a participant in the interview you will have the opportunity to review the transcripts of the interview and may withdraw any or all portions of the responses that you provided.

4. The researcher will keep all information collected in a secure location and only the researcher will have access to interview notes or other original data. All information will be kept strictly confidential by describing the site only in general terms or through the use of a non-identifiable reference code. All data identifying site or participant will be destroyed after five years.

5. Excerpts from the interviews and focus groups may be part of presentations, research reports, program evaluation reports, book chapters, or journal articles but anonymity will be maintained as that under no circumstances will name or identifying characteristics be used. 
6. All personal information collected for the evaluation of the Parenting Plus program is protected by the Protection of Privacy provisions of the Freedom of Information and Protection of Privacy Act and the Personal Health Information Act.

7. A copy of the consent form will be provided for your records.

I would be grateful if you would sign this form to indicate that you understand the nature of the research, the role of the investigator, your role and rights as participant and that you consent to participate in this evaluation. Should you have further questions or wish to contact me for any reason I can be telephoned at (306) 966-5258, or E Mail: dbm130@mail.usask.ca. Questions or concerns may also be directed to Dr. Vicki Schwean, Professor, Associate Head, Department of Educational Psychology and Special Education, College of Education, University of Saskatchewan; Telephone (306) 966-5246.

Participant Signature

Researcher Signature
Date

Date 


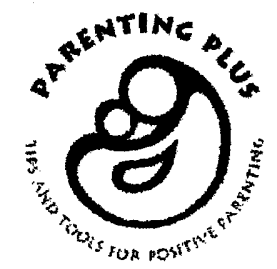

\section{FOCUS GROUP CONSENT FORM AND STATEMENT OF CONFIDENTIALITY}

We invite parents of the Parenting Plus program to participate in the evaluation of the program. The evaluation is of minimal risk and the purpose of the evaluation is to see:

- How this program makes a difference for the family and child

- How Pipestone's children and families are doing over time

- How well children's programs are working

- How we could improve the children's programs

- What other programs are needed

The evaluation is being conducted through the Pipestone Health District and the University of Saskatchewan. David Mykota, an Educational Psychology and Special Education Graduate student at the University of Saskatchewan is undertaking the evaluation. The title of the project is The Implementation Evaluation of Parenting Plus, the Early Childhood Development Program of the Pipestone Health District.

Your role as a participant in the evaluation will be to participate in a focus group session. The focus group would be arranged at a centralized location and will take three hours to complete.

As a participant in the focus group you have definite rights:

1. Your participation in any focus group is completely voluntary.

2. You are free to refuse to answer any question at any time and you are free to withdraw from the evaluation, at any time, without penalty.

3. As a participant in the focus group you understand that there could be violations of your privacy. To prevent violations of your own or others privacy, you have been asked not to talk about any of your own or other's private experiences that you would consider to be too private or revealing. You also understand that you have an obligation to respect the privacy of the other members of the group by not disclosing any personal information they share during our discussion.

4. As a participant in the focus group, if you so choose, every effort will be made to withdraw your responses, but that it may not be possible to withdraw your response in its entirety.

5. The researcher will keep all information collected in a secure location and only the researcher will have access to interview or focus group notes or other original data. All information will be kept strictly confidential by describing the site only in general terms or through the use of a non-identifiable reference code. All data identifying site or participant will be destroyed after five years. 
6. Excerpts from the interviews and focus groups may be part of presentations, research reports, program evaluation reports, book chapters, or journal articles but anonymity will be maintained as that under no circumstances will name or identifying characteristics will be used.

7. All personal information collected for the evaluation of the Parenting Plus program is protected by the Protection of Privacy provisions of the Freedom of Information and Protection of Privacy Act and the Personal Health Information Act.

8. A copy of the consent form will be provided for your records.

I would be grateful if you would sign this form to indicate that you understand the nature of the research, the role of the investigator, your role and rights as participant and that you consent to participate in this evaluation. Should you have further questions or wish to contact me for any reason I can be telephoned at (306) 966-5258, or E Mail: dbm130@mail.usask.ca. Questions or concerns may also be directed to Dr. Vicki Schwean, Professor, Associate Head, Department of Educational Psychology and Special Education, College of Education, University of Saskatchewan; Telephone (306) 966-5246.

These requirements to protect your personal information and personal health information apply to everyone involved in the collection and preparation of information for Parenting Plus evaluation.

Parent signature

Home Visitor signature

Researcher's signature

\section{Date}

Date

Date 


\section{PARENTING PLUS}

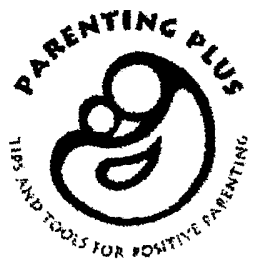

Pipestone Health District

\section{DATA/TRANSCRIPT RELEASE FORM}

I,

, have reviewed the complete transcript of my personal interview in this study, and acknowledge that the transcript accurately reflects what I said in my personal interview with David Mykota. I hereby authorize the release of this transcript to David Mykota to be used in the manner described in the consent form. I have received a copy of this Data/Transcript Release Form for my own records.

Participant

Researcher
Date

Date 
PARENTING PLUS

Pipestone Health District

\section{LETTER OF INVITATION}

Dear Parent,

I would like to invite you to attend our discussion at (name of location) in (town, address, etc.). We will be talking about your experiences with the Parenting Plus program. This is a study conducted by the University of Saskatchewan in conjunction with the Pipestone Health District to lean about how parent's home visiting programs are doing and how we could improve home visiting programs, like Parenting Plus. We want and need your opinions on these changes to help guide and inform future directions.

The discussion will last three hours and will involve about six to ten parents who also participate in Parenting Plus. Snacks will be provided and you will receive a twenty five dollar honorarium for participating. As well, up to a maximum of $\$ 25$ will be provided to you for transportation and babysitting costs. If you have difficulty finding transportation or babysitting, please contact your Family Support Worker.

Your participation (or non-participation) will not effect your ability to receive services from Parenting Plus.

I will be leading the discussion and if you have any further questions feel free to contact me at 306 966-5258.

Thank you so much for your assistance. Your participation will make a big difference in Parenting Plus.

Sincerely,

David Mykota. 


\section{PARENTING PLUS}

Pipestone Health District

\section{LETTER OF INVITATION}

\section{Dear Stakeholder,}

I would like to invite you to attend an interview at (name of location) in (town, address, etc.). I will be talking about your experiences with the Parenting Plus program. This is a study conducted by the University of Saskatchewan in conjunction with the Pipestone Health District to learn how the home visiting program is doing, how we could improve home visiting programs and what your role is in Parenting Plus. We want and need your opinions on these important issues to help guide and inform future directions for early intervention services.

A minimum of one to a maximum of three interview sessions are planned, lasting up to two hours each. They will be arranged at a time and location that is convenient for you. I will conduct the interview and you are free to refuse to answer any question at any time and you may withdraw all or any of your responses. Furthermore, all information collected by the evaluation of the Parenting Plus program is protected by the Protection of Privacy provisions of the Freedom of Information and Protection of Privacy Act and the Personal Health Information Act.

If you have any further questions please feel free to contact me at $306966-5258$.

Thank you so much for your assistance. Your participation will make a big difference in Parenting Plus.

Sincerely,

David Mykota. 
Appendix 6 
1. Background Information: name, position, length of employment in position, gender, and educational background.

2. Describe your role as it relates to the Parenting Plus Program of the Pipestone Health District.

3. What is your understanding of how Parenting Plus operates in the Pipestone Health District, in terms of target group, objectives, geographic area served, connections to other agencies in planning service and specific programming?

4. Could you describe how you first became involved in the program (who approached you, what was your understanding of what was being requested, what was your reaction)

5. How do you believe this program has an effect on services that were being delivered to the target group? (changed, enhanced, or new services being developed)

6. What resources were anticipated for full implementation?

-What staff competencies and roles were anticipated?

-What were the original intended time lines for implementation?

-What potential threats to implementation were anticipated during design?

7. What do you see as your primary responsibilities and have these responsibilities changed since the inception of the program? (how, why, or why not)

8. Describe your working relationship with the Pipestone Health District and the Parenting Plus program staff and what factors, positive or negative contributed to your ability to work together.

9. What has been learned about implementation of this specific program that might inform similar efforts elsewhere?

-As the program has been implemented, what model has emerged?

-To what extent and in what ways was the original implementation feasible?

-How stable and standardized has the implementation become both over time?

-To what extent is the program amenable to implementation elsewhere? What aspects of implementation are situational? What aspects are likely generalizable?

-What are the start up and continuing costs of implementation?

-Has implementation proved sufficiently effective and consistent that the program merits consideration?

10. Describe what you feel are the successes that can be attributed to this program?

Describe what you believe may prevent the program from developing its full potential as an early intervention service? (unexpected or negative processes or outcomes for clients, professionals, or community)

11. What will contribute to the programs success in the future?

12. Additional comments? 
1. Background Information: name, position, length of employment in position, gender, and educational background.

2. Describe your role in the Parenting Plus Program of the Pipestone Health District.

3. What factors led to the creation of the program in the Pipestone Health District?

-What was originally proposed and intended for implementation?

-What needs assessment or situation analysis informed program design?

-What was the programs expected model?

-What theory and assumptions under girded the proposed model, if any?

-Who has a stake in the program being implemented as proposed and originally designed?

4. What resources were anticipated for full implementation?

-What staff competencies and roles were anticipated?

-What were the original intended time lines for implementation?

-What potential threats to implementation were anticipated during design?

5. Is the governance structure put in place and did it provide the support and linkages required?

-Are there agreements with First Nations and Health Districts in place?

-Is there an advisory committee in place, who are the members and what are their roles?

-Is there a coordinator in place and what is their role?

-Are the family workers in place, who are their members and what are their roles?

6. Were procedures identified and implemented so those members of the designated population were given the opportunity to access the program?

-Who are completing the screens and how are they being sent to Parenting Plus?

-Are all families identified as high risk being assessed?

-Is there a referral process and who is making the referrals?

-Who is doing the family assessment after a referral is made and what does this entail?

-What changes must be made to reach the intended families more effectively?

7. How has Parenting Plus been implemented and managed?

-What are the programs key characteristics as perceived by you?

-What are the characteristics of program participants and how do those compare to the intended target population for the program?

-What were the procedures identified and implemented to track cases and gather information for case management as well as program development and evaluation purposes?

-What reports are being compiled and how is this information being communicated?

-What assumptions have proven true? What assumptions are problematic?

8. What do participants actually do in the program?

-What are their primary activities (in detail)? What do they experience?

-What were the specific strategies used to work with/interact with or involve participants?

-What do participants like and dislike?

-What are their perceptions of what is working and not working?

-Do the participants know what they're supposed to accomplish as participants?

-Do the participants buy into the programs goals and intended outcomes?

-How are the opportunities for control by the parent and attainment of their goals increased?

-How was success attained in the relationship between the parent and FSW? 
-What were the challenges faced by the parent in attaining success?

-What prevented the Family Support Worker from overcoming challenges to the client's success?

9. What are the short-term effects of Parenting Plus?

-How do actual resources, staff competencies, and experiences, and time lines compare to what was expected? -What is working as expected? What is not working as expected? What challenges and barriers have emerged? How has staff responded to those challenges and barriers?

-How have the families benefited or not benefited from the services provided by Parenting Plus?

-Were procedures identified and implemented to link clients with other community resources and supports?

-What lessons have been learned about the initial planned program design?

-How should these lessons be utilized in continually revising the original plan?

-Do changes in the program design reflect these lessons or other unrelated factors?

-How can we better connect program design changes to documented implementation lessons?

10. What has been learned about implementation of this specific program that might inform similar efforts elsewhere?

-As the program has been implemented what model has emerged?

-To what extent and in what ways was the original implementation feasible?

-How stable and standardized has the implementation become both over time?

-To what extent is the program amenable to implementation elsewhere? What aspects of implementation are situational? What aspects are likely generalizable?

-What are the start up and continuing costs of implementation?

-Has implementation proved sufficiently effective and consistent that the program merits consideration?

11. Additional comments? 
1. Background Information: name, position, length of employment in position, gender, and educational background.

2. Describe your role in the Parenting Plus Program of the Pipestone Health District.

3. Could you describe how the Parenting Plus Program operates in the Pipestone Health District, in terms of target group, objectives, geographic area served, connections to other agencies in planning service and specific programming?

4. Is the governance structure put in place and did it provide the support and linkages required?

-Are agreements with First Nations and Health Districts in place?

-Is there an advisory committee in place, who are the members and what are their roles?

-Are the family workers in place, who are their members and what are their roles?

5. What procedures were identified and implemented so those members of the designated population were given the opportunity to access the program?

-Who are completing the screens and how are they being sent to Parenting Plus?

-Are all families identified as high risk being assessed?

-Is there a referral process and who is making the referrals?

-Who is doing the family assessment after a referral is made and what does this entail?

-What changes must be made to reach the intended families more effectively?

6. How has Parenting Plus been implemented and managed?

-What are the programs key characteristics as perceived by you?

-What are the characteristics of program participants and how do those compare to the intended target population for the program?

-What were the procedures identified and implemented to track cases and gather information for case management as well as program development and evaluation purposes?

-What reports are being compiled and how is this information being communicated?

-What assumptions have proven true? What assumptions are problematic?

7. What do participants actually do in the program?

-What are their primary activities (in detail)? What do they experience?

-What were the specific strategies used to work with/interact with or involve participants?

-What do participants like and dislike?

-What are their perceptions of what is working and not working?

-Do the participants know what they're supposed to accomplish as participants?

-Do the participants buy into the programs goals and intended outcomes?

-How are the opportunities for control by the parent and attainment of their goals increased?

-How was success attained in the relationship between the parent and FSW?

-What were the challenges faced by the parent in attaining success?

-What prevented the Family Support Worker from overcoming challenges to the client's success?

8. What are the short-term effects of Parenting Plus?

-How do actual resources, staff competencies, and experiences, and time lines compare to what was expected?

-What is working as expected? What is not working as expected? What challenges and barriers have emerged? How has staff responded to those challenges and barriers?

-How have the families benefited or not benefited from the services provided by Parenting Plus?

-Were procedures identified and implemented to link clients with other community resources and supports? 
-What lessons have been learned about the initial planned program design?

-How should these lessons be utilized in continually revising the original plan?

-Do changes in the program design reflect these lessons or other unrelated factors?

-How can we better connect program design changes to documented implementation lessons?

9. What has been learned about implementation of this specific program that might inform similar efforts elsewhere?

-As the program has been implemented, what model has emerged?

-To what extent and in what ways was the original implementation feasible?

-How stable and standardized has the implementation become both over time?

-To what extent is the program amenable to implementation elsewhere? What aspects of implementation are situational? What aspects are likely generalizable?

-Has implementation proved sufficiently effective and consistent that the program merits consideration?

10. Additional comments? 
1. Background Information: name, position, length of employment in position, gender, and educational background.

2. Describe your role in the Parenting Plus Program of the Pipestone Health District.

3. Could you describe how the Parenting Plus Program operates in the Pipestone Health District, in terms of target group, objectives, geographic area served, connections to other agencies in planning service and specific programming?

4. Is the governance structure put in place and did it provide the support and linkages required?

-Are agreements with First Nations and Health Districts in place?

-Is there an advisory committee in place, who are the members and what are their roles?

-Are the family workers in place, who are their members and what are their roles?

5. What procedures were identified and implemented so those members of the designated population were given the opportunity to access the program?

-Who are completing the screens and how are they being sent to Parenting Plus?

-Are all families identified as high risk being assessed?

-Is there a referral process and who is making the referrals?

-Who is doing the family assessment after a referral is made and what does this entail?

-What changes must be made to reach the intended families more effectively?

6. How has Parenting Plus been implemented and managed?

-What are the programs key characteristics as perceived by you?

-What are the characteristics of program participants and how do those compare to the intended target population for the program?

-What were the procedures identified and implemented to track cases and gather information for case management as well as program development and evaluation purposes?

-What reports are being compiled and how is this information being communicated?

-What assumptions have proven true? What assumptions are problematic?

7. What do participants actually do in the program?

-What are their primary activities (in detail)? What do they experience?

-What were the specific strategies used to work with/interact with or involve participants?

-What do participants like and dislike?

-What are their perceptions of what is working and not working?

-Do the participants know what they're supposed to accomplish as participants?

-Do the participants buy into the programs goals and intended outcomes?

-How are the opportunities for control by the parent and attainment of their goals increased?

-How was success attained in the relationship between the parent and FSW?

-What were the challenges faced by the parent in attaining success?

-What prevented the Family Support Worker from overcoming challenges to the client's success?

8. What are the short-term effects of Parenting Plus?

-How do actual resources, staff competencies, and experiences, and time lines compare to what was expected?

-What is working as expected? What is not working as expected? What challenges and barriers

have emerged? How has staff responded to those challenges and barriers?

-How have the families benefited or not benefited from the services provided by Parenting Plus?

-Were procedures identified and implemented to link clients with other community resources and supports? 
-What lessons have been learned about the initial planned program design?

-How should these lessons be utilized in continually revising the original plan?

-Do changes in the program design reflect these lessons or other unrelated factors?

-How can we better connect program design changes to documented implementation lessons?

9. What has been learned about implementation of this specific program that might inform similar efforts elsewhere?

-As the program has been implemented, what model has emerged?

-To what extent and in what ways was the original implementation feasible?

-How stable and standardized has the implementation become both over time?

-To what extent is the program amenable to implementation elsewhere? What aspects of implementation are situational? What aspects are likely generalizable?

-Has implementation proved sufficiently effective and consistent that the program merits consideration?

10. Additional comments? 